\title{
The Southern Ocean Meridional Overturning Circulation as Diagnosed from an Eddy Permitting State Estimate
}

by

Matthew R. Mazloff

M.S. in Physics, University of Vermont, 2002

M.S. in Physical Oceanography, MIT-WHOI Joint Program, 2006 Submitted in partial fulfillment of the requirements for the degree of Doctor of Philosophy in Physical Oceanography

at the

MASSACHUSETTS INSTITUTE OF TECHNOLOGY

and the

WOODS HOLE OCEANOGRAPHIC INSTITUTION

September 2008

(c) Matthew R. Mazloff, MMVIII. All rights reserved.

The author hereby grants to MIT and WHOI permission to reproduce and distribute publicly paper and electronic copies of this thesis document in whole or in part.

Author ................................................

Joint Program in Physical Oceanography - Massachusetts Institute of Technology / Woods Hole Oceanographic Institution August 1, 2008

Certified by .

Carl Wunsch

Cecil and Ida Green Professor of Physical Oceanography Thesis Supervisor Accepted by

Raffaele Ferrari Chair, Joint Committee for Physical Oceanography 


\title{
The Southern Ocean Meridional Overturning Circulation as Diagnosed from an Eddy Permitting State Estimate
}

\author{
by \\ Matthew R. Mazloff \\ Submitted to the Joint Program in Physical Oceanography - Massachusetts \\ Institute of Technology / Woods Hole Oceanographic Institution \\ on August 1, 2008, in partial fulfillment of the \\ requirements for the degree of \\ Doctor of Philosophy in Physical Oceanography
}

\begin{abstract}
A modern general circulation model of the Southern Ocean with one-sixth of a degree resolution is optimized to the observed ocean in a weighted least squares sense. Convergence to the state estimate solution is carried out by systematically adjusting the control variables (atmospheric state and initial conditions) using the adjoint model. A cost function compares the model state to in situ observations (Argo float profiles, CTD synoptic sections, SEaOS instrument mounted seal profiles, and XBTs), altimetric observations (ENVISAT, GEOSAT, Jason, TOPEX/Poseidon), and other data sets (e.g. infrared and microwave radiometer observed sea surface temperature and NSIDC sea-ice concentration). Costs attributed to control variable perturbations ensure a physically realistic solution. The state estimate is found to be largely consistent with the individual observations, as well as with integrated fluxes inferred from previous static inverse models.

The transformed Eulerian mean formulation is an elegant way to theorize about the Southern Ocean. Current researchers utilizing this framework, however, have been making assumptions that render their theories largely irrelevant to the actual ocean. It is shown that theories of the overturning circulation must include the effect of pressure forcing. This is true in the most buoyant waters, where pressure forcing overcomes eddy and wind forcing to balance a poleward geostrophic transport and allows the buoyancy budget to be closed. Pressure forcing is also lowest order at depth. Indeed, the Southern Ocean's characteristic multiple cell overturning is primarily in geostrophic balance. Several other aspects of the Southern Ocean circulation are also investigated in the thesis, including an analysis of the magnitude and variability of heat, salt, and volume inter-basin transports.
\end{abstract}

Thesis Supervisor: Carl Wunsch

Title: Cecil and Ida Green Professor of Physical Oceanography 


\section{Acknowledgments}

Carl Wunsch has provided me with guidance, inspiration, resources, and support. He has been, and I'm sure will continue to be, a wonderful advisor. I am grateful for Carl's dedication. I am also grateful for Patrick Heimbach, who made this thesis possible by guiding me through SOSE's production. Working with Carl, Patrick, Gael, Jean-Michel, and the rest of the ECCO project has been more than a great learning experience; it has been fun, and for this I am extremely appreciative.

The analysis of the meridional overturning circulation (Chapter 4) was carried out with Raffaele Ferrari. Raf was vital in both the formulation of the problem and the interpretation of the results. He made learning about the Southern Ocean through analysis of the state estimate exciting. Researchers like Carl, Patrick, and Raf are amazingly enthusiastic and talented. Being surrounded by such people has been a wonderful experience, and I feel both lucky and thankful for their time.

The thesis was also greatly improved through conversations with Mary-Louise Timmermans, John Toole, and other researchers. Besides being very informative, these interactions were enjoyable. I look forward to future collaborations.

Being a student in the MIT-WHOI Joint Program is a great experience. The administrators, fellow students, and professors all deserve recognition in making this thesis work possible and, for the most part, fun. At the risk of being neglectful, I will give a short list of people who are deserving of special thanks. Thanks to the best office mates in the world: Bea, Gerbi, Holly, Stephanie, and Yowchi. These friends, along with Alex, Ariane, Evgeny, Jake, Jinbo, Peter, Rosey the Rose, Ryan, Sutherland, Tatiana, and the man@mit have kept me sane. Many other people who have contributed in some way to this work are not listed, largely for brevity. I hope they accept my apologies, and also realize that I do appreciate their friendship.

I would like to thank the friendly people at SDSC. Their eagerness to help takes the complication out of supercomputing. Without their support this work would not have been possible.

Lastly I would like to thank my wonderful family who are always supportive of 
me, especially Kendall and Stella.

This work was supported by CalTech - Jet Propulsion Lab contract \#1205624 (Global Oceans Dynamics and Transports). Support for my first 2 years in the MITWHOI Joint Program came from NSF awards \#OCE-9901654 (Research in Linear and Nonlinear Waves and Ocean Circulation Theory). I was also supported for two months by NSF awards \#OCE-0223434. Computational resources were provided by San Diego Supercomputer Center under proposal number MCA06N007. 


\section{Contents}

1 Introduction 11

1.1 Synopsis of Thesis . . . . . . . . . . . . . . . . . . 13

1.2 A Model-Observation Least-Squares Optimization . . . . . . . . . . 14

1.3 The Forward Model . . . . . . . . . . . . . . . . . . . . . . . . . 16

1.3.1 Forward Model Inputs . . . . . . . . . . . . . . . . . . 17

1.3.2 Forward Modeling Efforts . . . . . . . . . . . . . . . . 19

1.4 The Adjoint Model . . . . . . . . . . . . . . . . . . . . . . . 20

1.5 Gradient Descent . . . . . . . . . . . . . . . . . . . . . . . . 21

2 Observations and Consistency of the State Estimate 23

2.1 Observations . . . . . . . . . . . . . . . . . . . 24

2.1 .1 Altimetry . . . . . . . . . . . . . . . . 24

2.1 .2 Climatology . . . . . . . . . . . . . . . . 26

2.1 .3 In Situ Measurements . . . . . . . . . . . . . . . . 26

2.1.4 Sea Surface Temperature . . . . . . . . . . . . . . . 27

2.1 .5 Sea Ice . . . . . . . . . . . . . . . . . . . . . . . . . . 28

2.1 .6 Uncertainty . . . . . . . . . . . . . . . . . . . . . 28

2.2 The Control Vector . . . . . . . . . . . . . . . . . . . . . . . . . . . . . 29

2.3 Model-Observation Fit . . . . . . . . . . . . . . . . . . . . . 31

2.3 .1 In Situ Measurements . . . . . . . . . . . . . . . . . . . 32

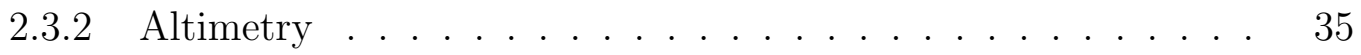

2.3.3 Sea Surface Temperature . . . . . . . . . . . . . . . . 37

$2.3 .4 \quad$ Sea Ice . . . . . . . . . . . . . . . . . . . . . . . . . . 38 
2.3 .5 Summary . . . . . . . . . . . . . . . . . . . . 39

3 Analysis of a Southern Ocean State Estimate 43

3.1 Introduction . . . . . . . . . . . . . . . . . . . . . . 43

3.1 .1 Water Masses . . . . . . . . . . . . . . . . . . 45

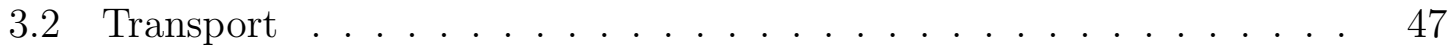

3.2.1 Vertically Integrated Transport Streamfunction _ . . . . . . 47

3.2.2 Freshwater and Temperature Flux . . . . . . . . . . 51

3.2 .3 Ekman Transport . . . . . . . . . . . . . . . . . 56

3.2.4 Meridional Overturning Streamfunction . . . . . . . . . 58

3.3 Rate of Wind Work . . . . . . . . . . . . . . . . . . 65

3.4 Conclusions . . . . . . . . . . . . . . . . . . . . . . . 68

4 Dynamics of the Meridional Overturning Circulation 69

4.1 Theoretical Review . . . . . . . . . . . . . . . . . . . . 70

4.1.1 Sverdrupian Theories: A Southern Ocean Gyre . . . . . . . 70

4.1 .2 Zonal Channel Theories . . . . . . . . . . . . . . . . . 72

4.1.3 The Residual Meridional Overturning Circulation . . . . . . 76

4.2 Derivation of the Zonal Momentum Balance in Isopycnal Space . . . 79

4.3 Results . . . . . . . . . . . . . . . . . . . . . . . 84

4.3 .1 Introduction . . . . . . . . . . . . . . . . 84

4.3.2 The Ageostropohic Residual Circulation . . . . . . . . . 87

4.3.3 The Geostropohic Residual Circulation . . . . . . . . . . . 89

4.3.4 The Total Residual Circulation _. . . . . . . . . . . 92

4.3.5 Buoyancy Fluxes . . . . . . . . . . . . . . . 95

$4.3 .6 \quad$ Model Drift . . . . . . . . . . . . . . . . . . . . . 96

4.3.7 Surface Buoyancy Fluxes . . . . . . . . . . . . . . . . . . . 99

4.3.8 The Eddy Potential Vorticity Flux _ . . . . . . . . . . 100

4.4 Summary and Conclusions . . . . . . . . . . . . . . . . 103 
5 Conclusions and Future Work

5.1 The Southern Ocean State Estimate . . . . . . . . . . . . . . . . 109

5.2 Analysis of the Zonal Momentum Balance . . . . . . . . . . . . . 111 


\section{Chapter 1}

\section{Introduction}

The mid- and high-latitude Southern Hemisphere oceans play a significant role in determining the global climate. These waters are responsible for linking the Atlantic, Indian, and Pacific Oceans. They are the world's largest oceanic sink of atmospheric $\mathrm{CO}_{2}$ (Patra et al., 2005), and atmospheric wind energy (Wunsch, 1998). The signature of the water masses formed in this region are found throughout the global oceans (Marsh et al., 2000). Yet despite the Southern Ocean's significance, the circulation and variability of these waters are poorly understood. ${ }^{1}$

One reason for the lack of understanding is a deficiency of historical observations in this remote area. Southern Ocean observations were rare prior to the Eltanin cruises in the early 1960s (Nierenberg et al., 1970). Though becoming more frequent after 1962, observations were often focused on specific regions, for example the Drake Passage. Coverage dramatically increased in both space and time with the introduction of high precision satellite altimeters in the early 1990's, and again in 2002 with the introduction of hundreds of autonomous floats through the Argo program (Gould and the Argo Steering Team, 2004). In 2003 the SEaOS program began mounting CTD instruments on elephant seals to gather data in the polar regions. There has been an unprecedented influx of Southern Ocean observations over the last several

\footnotetext{
${ }^{1}$ For purposes of the Antarctic Treaty, the International Hydrographic Organization has defined the Southern Ocean to extend from Antarctica north to $60^{\circ} \mathrm{S}$. The region of study in this thesis is the oceans south of latitude $25^{\circ} \mathrm{S}$, which, for ease, will be referred to collectively as the Southern Ocean.
} 
years.

Even with available observations, the task of describing the Southern Ocean circulation is not trivial. The Southern Ocean is extremely energetic, and eddies play a large role in the dynamics. The signals these eddies leave in the observations must be diagnosed (Lee and Coward, 2003). Eddy dynamics occur on the order of the Rossby deformation radius, which ranges from order $30 \mathrm{~km}$ at $40^{\circ} S$ to less than 10 $\mathrm{km}$ near Antarctica (Chelton et al., 1998). Modeling the Southern Ocean is made difficult by these facts; models must either have high-resolution or rely heavily on eddy parameterizations.

Large data sets of Southern Ocean observations are now available. The task is to use these disparate and diverse forms of data in a way that facilitates physical understanding of ocean processes. The consortium for Estimating the Circulation and Climate of the Ocean (ECCO) was established with the goal of combining a physically realistic general circulation model with observations to produce quantitative accounts of the global ocean state (see http://www.ecco-group.org). ECCO has had difficulty estimating the state of the Southern Ocean, partly because the global models they have used are of too coarse a resolution to capture the meso-scale dynamics in this highly energetic region. Working with the ECCO group I have produced and analyzed an eddy permitting regional state estimate of the Southern Ocean. That a multiyear high-resolution model solution of this energetic part of the world oceans can be brought into consistency with observations using the adjoint method is one significant result of this thesis.

The circulation of the Southern Ocean is largely wind driven. It is estimated that more than $60 \%$ of the energy input from the wind to the world's oceans occurs polewards of $30^{\circ} \mathrm{S}$ (see Section 3.3). The majority of this transfer results from the large zonal wind-stress component. How the Southern Ocean reacts to this large zonal momentum flux is a matter of debate (e.g. Warren et al. (1998); Olbers et al. (2004)). The substantial wind forcing, along with a lack of a complete meridional boundary, results in the Southern Ocean's most prominent feature being a massive eastward oceanic transport circumnavigating Antarctica. This transport, which is 
one of the largest in all the world's oceans, is known as the Antarctic Circumpolar Current (ACC). Significant bottom velocities in this current system cause it to be greatly affected by topography (Tansley and Marshall, 2001). Ridge structures, such as the Scotia Ridge found to the east of the Drake Passage, alter the large-scale flow of the ACC and cause a local increase in small-scale turbulence (Garabato et al., 2004).

The Southern Ocean currents, including the ACC, are sketched in schematic form in Figure 1-1 (from Rintoul et al., 2001). Associated with these current systems is a meridional overturning circulation (MOC). This MOC, which affects the waters of all the major basins of the world's oceans, is poorly understood. There are many theories as to what determines the structure of the overturning. These theories, however, rely heavily on untested assumptions, and how relevant they are to the actual Southern Ocean MOC is questionable. In this thesis a state estimate is used to analyze the force balance of the overturning circulation, and by doing so tests commonly made theoretical assumptions. It is found that the common assumption of a negligible geostrophic component to the MOC is not appropriate.

\section{$1.1 \quad$ Synopsis of Thesis}

The remainder of this chapter describes the machinery used to carry out the state estimate. This includes a discussion of the formulation of the problem, and the computer models and software used to carry it out. Chapter 2 discusses the observations used in the optimization. This chapter includes analyses of how the optimization has changed the initial and surface boundary conditions, and of how well the state estimate solution fits the data. These first two chapters are largely procedural and somewhat brief; one is referred to my Master's thesis, Mazloff (2006), and the references therein for more detail.

Chapter 3 uses the state estimate to give a descriptive analyses of the Southern Ocean transports. Chapter 4 is devoted to understanding the force balance of the Southern Ocean meridional overturning circulation. The chapter begins with a section 


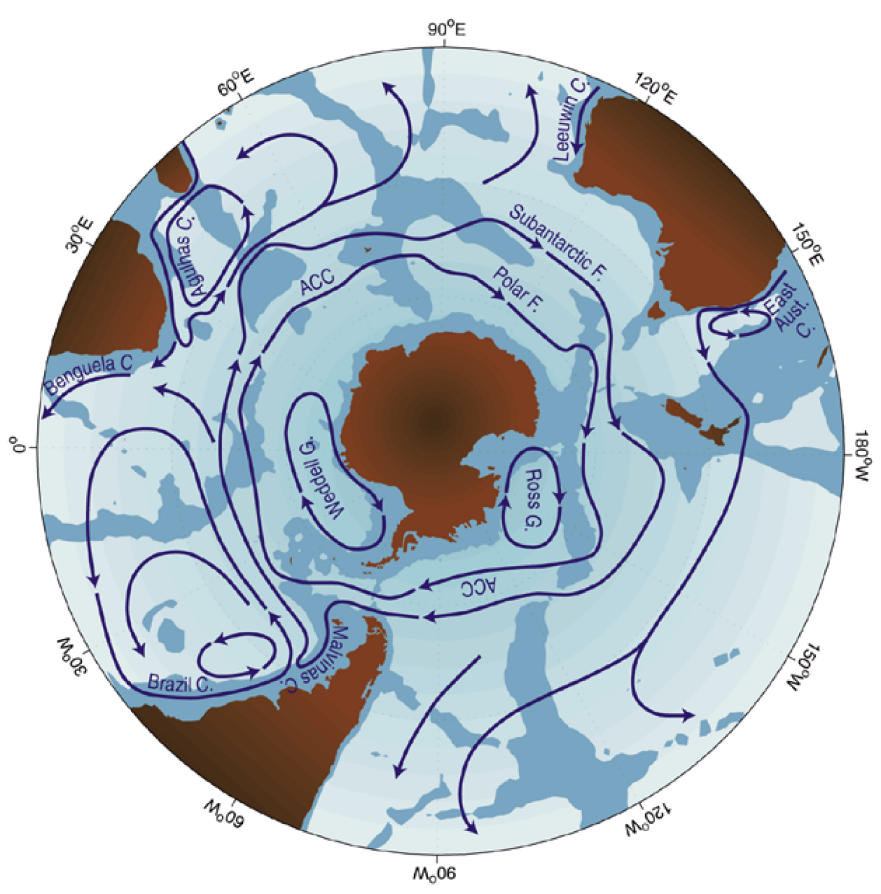

Figure 1-1: A schematic of the major Southern Ocean currents from Rintoul et al. (2001). Depths shallower than 3,500m are shaded. The poleward flanks of the cyclonic subtropical gyres are shown to the north. The anti-cyclonic polar gyres are adjacent to Antarctica. Between the subtropical and polar gyres is the massive Antarctic Circumpolar Current system (ACC). The ACC experiences significant meridional excursions (e.g. the Malvinas (or Falkland) Current east of South America) as it circumnavigates the pole.

reviewing the current theories on the Southern Ocean circulation. In the following two sections the zonal momentum balance of the seasonal MOC is solved for and analyzed. Chapter 5 gives a summary of the conclusions and results of the thesis. Suggestions for future work are also given in this final chapter.

\subsection{A Model-Observation Least-Squares Optimiza- tion}

An ocean circulation model can be used to determine a physically realistic, timevarying, ocean state (velocity, temperature, salinity, and sea level). Though physically realistic, this calculated state may be quite different from the observed ocean. To monitor and understand our World's oceans, one desires that the calculated state is 
as close to reality as possible. For this thesis, a modern ocean circulation model state has been brought considerably closer to consistency with observations by formulating the problem into a weighted least-squares optimization with Lagrange multipliers. This well documented model-data synthesis procedure is widely referred to in this oceanographic context as the adjoint method (Wunsch, 2006; Wunsch and Heimbach, 2006).

It is assumed here that the model used, which is described in Section 1.3, has little uncertainty associated with its dynamics (i.e. uncertainty in the parameterizations of small scale ocean processes is neglected in the present state estimate). It is acknowledged, however, that there is a significant level of uncertainty in the model inputs. Perturbations to these input fields control the model solution, and are therefore referred to as "controls". The optimization process is to iteratively solve for the controls in order to find a model trajectory that is within the combined model and data uncertainty. The trajectory converged to is the state estimate.

The performance of the state estimate is reduced to one number through the cost function. This function is a sum of squared model-data misfit terms and squared control vector magnitude terms. Every term is normalized by its prescribed uncertainty. The task at hand is to minimize this weighted sum of squares. Reduction of the cost to an acceptable value, however, is not the only requirement for a successful state estimate. One also requires the residual misfit to be random white-noise whose square follows a $\chi_{1}^{2}$ distribution. The remaining misfit of the state estimate calculated in this work is evaluated in Section 2.3. For a thorough presentation of the cost function used, and a description of the method of Lagrange multipliers please see Mazloff (2006) and the references therein. ${ }^{2}$

\footnotetext{
${ }^{2}$ More observations have been gathered since Mazloff (2006), and these additional model constraints have been implemented.
} 


\subsection{The Forward Model}

The MIT Ocean General Circulation Model (MITgcm; Marshall et al., 1997) is used to solve the Boussinesq hydrostatic primitive equations on a "C" grid (Arakawa and Lamb, 1977). Computational resources are limited, and thus so too is model resolution. The model is unable to reproduce ocean dynamics occurring on scales on the order of the grid-spacing, and the effect of these small-scale processes on resolved dynamics must be parameterized. The parameterizations used in this study include the Gent-McWilliams-Redi eddy parameterization, and the non-local K-profile vertical mixing parameterization (KPP: Large et al. (1994)). A sea-ice thermodynamical model, based on Hibler (1980), is employed. For more information on the MITgcm see Adcroft et al. (2006).

The Gent-McWiliams-Redi parameterization considers the effect of geostrophic eddies as two separate processes, and thus involves two separate parameterizations. The first parameterization represents the mixing of tracers along isopycnals and takes the form of a diffusive operator acting parallel to density surfaces (Redi: Redi (1982)). The second parameterization (Gent-McWiliams: Gent and McWilliams (1990)) represents an adiabatic advection by eddies. The advective velocity used in this parameterization is a function of the slope of the density surfaces. For the Southern Ocean state estimate the along isopycnal diffusive and advective flux coefficients are both set to $10 \mathrm{~m}^{2} \mathrm{~s}^{-1}$. This relatively small coefficient (compared to more conventional values: Gent et al. (2002); Ferreira et al. (2005)) reflects the fact that the state estimate resolves much of the eddy scales.

The KPP scheme is implemented to account for vertical mixing caused by both wind stirring and convection (Large et al., 1994). In this scheme the vertical fluxes are parameterized as $\overline{w^{\prime} c^{\prime}}=-\kappa_{c}\left(\partial \bar{c} / \partial z-\gamma_{c}\right)$ where $c$ is some tracer quantity (temperature and salinity in this state estimate). An overbar denotes the model's resolved flow, and primes denote dynamics that are, for the most part, unresolved. The turbulent diffusivity, $\kappa_{c}$, is given as a function of the turbulent velocity scale, $w^{*}$, and a nondimensional shape function, $\mathcal{G}(\sigma)$, such that $\kappa_{c}=h w^{*}(\sigma) \mathcal{G}\{\sigma)$. Here $\sigma=z / h$, where 
$h$ is the depth of the turbulent boundary layer determined as the location where the bulk Richardson number exceeds a critical value. $\gamma_{c}$ is the non-local flux term implemented to represent the fact that scalar fields may be largely homogeneous in convective boundary layers, yet fluxes may still be finite. The MITgcm modeling community has had success representing proper physics with this parameterization (Stammer et al., 2003; Gebbie, 2004).

The model is constructed in spherical coordinates with $1 / 6^{0}$ horizontal resolution and 42 vertical levels of varying depth. The solution is solved for using a 900 second time step. In addition to the parameterization schemes discussed above, small scale diffusion and dissipation of salinity and temperature is represented via a harmonic operator in the vertical $\left(\kappa_{v} \partial^{2} / \partial z^{2}\right.$ with coefficient $\left.\kappa_{v}=10^{-5} m^{2} s^{-1}\right)$, and a bi-harmonic operator in the horizontal (the coefficient used was $10^{10} \mathrm{~m}^{4} \mathrm{~s}^{-1}$ ). Momentum diffusion and dissipation is represented via a Laplacian viscosity with horizontal coefficient $\nu_{h}=10 \mathrm{~m}^{2} \mathrm{~s}^{-1}$ and vertical coefficient $\nu_{v}=10^{-4} \mathrm{~m}^{2} \mathrm{~s}^{-1}$. No-slip boundary conditions are used with a linear bottom drag coefficient of $10^{-3} \mathrm{~s}^{-1}$. To further parameterize topographic interaction, and to help stabilize the model, the viscosity is increased to the values used in the adjoint model (see Section 1.4) in the 3 layers nearest the ocean floor. In terms of the diffusivity and viscosity coefficients selected, the set up is rather typical (e.g. Saunders and Thompson (1993); Gebbie (2004)).

\subsubsection{Forward Model Inputs}

The Southern Ocean bathymetry file was derived by first binning and averaging, on a $1 / 4^{0}$ grid, the high-resolution topography data (GTOPO30) of Smith and Sandwell (1997). These data cover the domain equatorward of $\approx 72^{0} S$. Topography for the remainder of the domain comes from the ETOP05 data set (NOAA, 1988). This $1 / 4^{0}$ field is then interpolated to fit the $1 / 6^{0}$ model grid. In this sense, the bathymetry used is slightly smoothed. Some degree of bathymetric smoothing is desirable in the absence of a correct bottom boundary layer parameterization (Penduff et al., 2002). The model is discretized on a finite volume grid with 42 depth levels, and employs partial cells such that a cell may be cropped to better fit the bathymetry Adcroft 


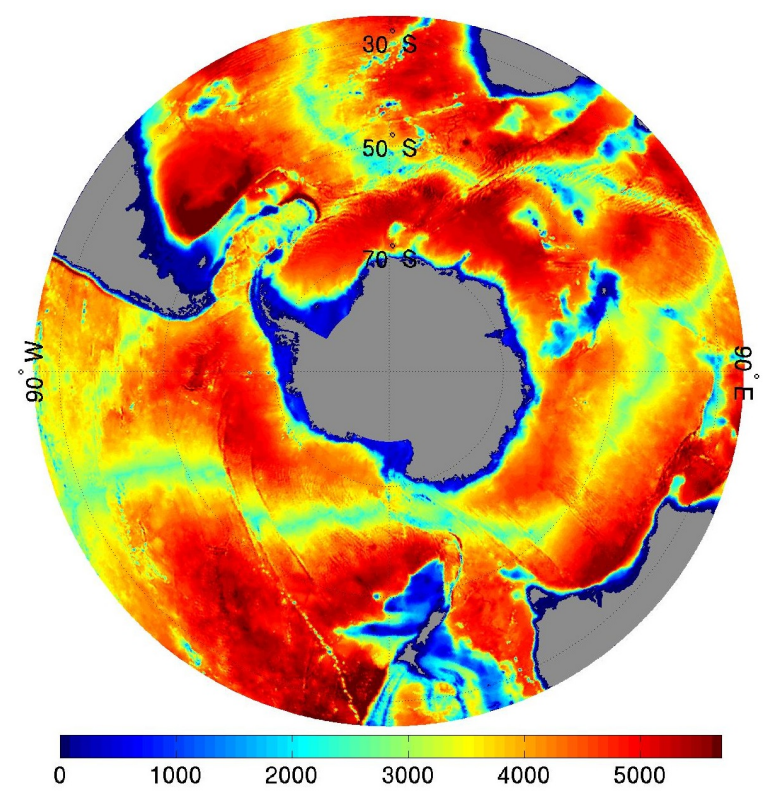

Figure 1-2: Model bathymetry [meters]

and Marshall (1998). The depth profile, exactly as represented by the model, is shown in Figure (1-2). Model bathymetry can greatly affect the flow field (Losch and Wunsch, 2003; Losch and Heimbach, 2007). Many modeling efforts, including the ECCO-GODAE production run, tune the bathymetry to ensure model transports are realistic in well observed ocean constriction points (e.g the Drake Passage and Florida Strait) (Lu et al., 2002). No tuning of the topography, or cropping of the ocean shelves, has been carried out in this present state estimate.

The initial estimate of the atmospheric state is obtained from a re-analysis of historical data produced in a joint effort between the National Centers for Environmental Prediction and the National Center for Atmospheric Research: the NCEP/NCAR Reanalysis Project (Kalnay and coauthors, 1996). The following fields, which are available at 6 hour intervals, are used to prescribe the atmospheric state:

- Air temperature [Kelvin] at 2 meters

- Specific humidity [kilogram water vapor / kilogram air] at 2 meters

- Zonal wind speed [meters / second] at 10 meters

- Meridional wind speed [meters / second] at 10 meters

- Precipitation [meters / second] 
- Short wave radiative flux [watts / meter $^{2}$ ]

- Long wave radiative flux [watts / meter $^{2}$ ]

A monthly river (freshwater) runoff climatology is also prescribed in the model. This climatology was determined by analyzing both observed river runoff, and the climatological difference between evaporation and precipitation over continents (Fekete et al., 2002). Appropriate momentum, heat, and mass fluxes are determined by comparing the prescribed atmospheric and modeled ocean states using bulk formulae (Large and Pond (1982); and see Adcroft et al. (2006) for more information).

The first guess initial conditions are derived by taking a one year spin-up of a $1^{o}$ global state estimate carried out over a similar time interval by Forget (2008). Forget's state estimate is also used to provide an open ocean boundary condition at the northern extent $\left(24.7^{\circ} \mathrm{S}\right)$ of this regional state estimate. Care was taken to ensure that volume transport was conserved when interpolating the $1^{\circ}$ state estimate fields into the higher resolution northern boundary condition.

Boundary regions are characterized by strong velocity gradients as the flow is brought to rest. These gradients often lead to significantly stronger turbulent dissipation and vertical motions than are found in the open ocean (Toole et al., 1994). Though the prescribed northern boundary condition is not one of vanishing velocity, boundary effects may still occur if the prescribed conditions are inconsistent with the interior circulation. Improperly prescribed northern boundary conditions can therefore lead to unphysical ocean interior features. Dealing with open boundary conditions in either a forward or adjoint model is a difficult task (Hoteit et al., 2005); to minimize this issue in such a large calculation the northern boundary condition is not adjusted in the optimization. Future work, however, should include this important control parameter.

\subsubsection{Forward Modeling Efforts}

Constraining an eddy-permitting forward model of the Southern Ocean to observations is a novel endeavor. Calculating an unconstrained solution, however, has been 
achieved. Early solutions came from the Fine Resolution Antarctic Model (FRAM: e.g. Saunders and Thompson (1993)), and more recent solutions from its predecessor, the Ocean Circulation and Climate Advanced Model (OCCAM: e.g. Lee et al. (2007)). Recent analysis has also been carried out on the $1 / 6^{0}$ Hallberg Isopycnal Model (HIM: e.g. Hallberg and Gnanadesikan (2006)). Other solutions are also available from various sources; to name a few: the Hybrid Coordinate Ocean Model (HYCOM: http://hycom.rsmas.miami.edu/index.shtml), a model for the Earth Simulator based on the Modular Ocean Model (MOM3: citeyuk04), the Australian Community Ocean Model (AusCOM: Bi et al. (2008)) based on MOM4, and the Center for Climate System Research (CCSR) Ocean Component model (COCO: Tanaka and Hasumi (2008)). An evaluation of how consistent any of these model solutions are with respect to the observed Southern Ocean has not been published.

\subsection{The Adjoint Model}

The efficient calculation of the adjoint code has been made feasible thanks to the existence of automatic differentiation (AD) tools. Giering and Kaminski (1998) have provided an AD tool, known as Transformation of Algorithms in Fortran (TAF), which determines the gradients of the forward model with respect to the control and state variables by rigorously applying the chain rule, line by line, to the forward code. Though TAF produces the exact adjoint of the forward model code, some packages are omitted from the adjoint model used in the optimization procedure due to model stability issues. The adjoint of the Gent-McWilliams-Redi parameterization and the KPP mixed layer parameterization are omitted. For the Gent-McWilliams-Redi parameterization this omission is rather insignificant, as the effect of this scheme is minimal because a small mixing coefficient has been chosen (see Section 3.2.4). There is a good deal of uncertainty in the choice of the coefficients used in these parameterizations, and it would be ideal to let the optimization solve for them. Omission of these packages, however, makes solving for the coefficients impossible.

These parameterization packages (Gent-McWilliams-Redi and KPP) were removed 
from the adjoint model due to stability issues. Similarly, the adjoint of the sea-ice model omitted from the model. Research to resolve the issues these packages pose is underway. To further ensure the adjoint model's stability, the model is run with higher viscosity and diffusivity coefficients (Hoteit et al., 2005). In the adjoint model, Laplacian viscosity and diffusivity coefficients are increased to $\nu_{h}=10^{3} \mathrm{~m}^{2} \mathrm{~s}^{-1}$ and $\kappa_{h}=10^{3} \mathrm{~m}^{2} \mathrm{~s}^{-1}$ in the horizontal, and $\nu_{v}=10^{-3} \mathrm{~m}^{2} \mathrm{~s}^{-1}$ and $\kappa_{v}=10^{-3} \mathrm{~m}^{2} \mathrm{~s}^{-1}$ in the vertical. A bi-harmonic horizontal viscosity of $10^{9} \mathrm{~m}^{4} \mathrm{~s}^{-1}$ is used.

Availability of the adjoint model code, whether exact or not, does not guarantee a successful optimization (Köhl and Willebrand, 2002; Hoteit et al., 2005). The findings of this work are that an inexact adjoint model can be used to reduce model-data misfit in an eddy permitting model, which is consistent with Gebbie et al. (2006) and Hoteit et al. (2005). The optimization behaved comparably to state estimates carried out at $1^{0}$ resolution. The quality of the Southern Ocean state estimate is evaluated in Chapter 2.

\subsection{Gradient Descent}

Substantial work in optimization theory (see, for example, Gill et al. (1986)) has allowed for this present optimization problem of considerable dimension (the cost function has over 2 billion terms) to be possible. The Lagrange multiplier method employed makes known the gradients of the cost function with respect to the controls, which increases the efficiency of search algorithms. For the optimization carried out here the quasi-Newtonian optimization algorithm of Gilbert and Lemaréchal (1989) was used. This search method uses stored gradients of the cost function from previous iterations to approximate the Hessian matrix. By only approximating the Hessian matrix, valuable second derivative (curvature) information is made available without an excessive computational demand. 


\section{Chapter 2}

\section{Observations and Consistency of the State Estimate}

The state of the Southern Ocean is estimated for the years 2005 and 2006 using the adjoint method. These years are both periods of positive Southern Annular Mode (SAM) index. The SAM index is defined as the difference in the normalized monthly zonal-mean sea level pressure between $40^{\circ} \mathrm{S}$ and $70^{\circ} \mathrm{S}$ (Gong and Wang, 1999; Nan and Li, 2003). The NCEP/NCAR reanalysis (Kalnay and coauthors, 1996) implies that the mean SAM index for 2005 was 0.36 with a monthly standard deviation of 1.44, and the mean SAM for 2006 was 1.08 with a monthly standard deviation of 1.49 . A positive SAM index is associated with cold sea surface temperature (SST) anomalies poleward of the ACC, and warm SST anomalies equatorward of the ACC. A positive index implies stronger than average westerly winds over the Southern Ocean, which results in an increased ACC transport. Much of what is known about the mean ACC transport comes from the International Southern Ocean Study (ISOS), which occurred between 1974 and 1981. These years were marked by large SAM index shifts. On average, however, they were years of negative SAM index. When comparing prior observational inferences to the current results, one should consider shifts in the SAM index.

The observations used to constrain the state estimate solution are described in Section (2.1), the control vector changes made to achieve a more consistent solution 
are discussed in Section (2.2), and the consistency with these observations is discussed in Section (2.3). Thirty-two iterations were carried out to attain the current state estimate solution. In this work two of these iterations have been evaluated: iteration 22 was used for the dynamical and transport analysis, and iteration 26 was used for the misfit analysis. The two iterations are qualitatively very similar, though iteration 22 has a $12 \%$ higher cost then iteration 26. The state estimate is a work in progress; neither iteration 22, iteration 26 , nor iteration 32 are converged solutions. It is shown in this chapter, however, that the solution that has been attained is useful for analysis.

It is difficult to quantify how much the cost was reduced, as the cost function was changed multiple times over the optimization. Since iteration 17, however, terms and weights in the cost function have not changed, and thus it is possible to quantify the progress of the optimization since this point. Since iteration 17 the cost has come down $31 \%$ (Figure 2-1). It is worth pointing out that to achieve this involved solving for over 700 million model input terms in order to control a 10 trillion term ocean state and reduce a 1.4 billion term cost function.

\section{$2.1 \quad$ Observations}

\subsubsection{Altimetry}

High precision satellite altimeters have been providing a wealth of observations since 1993. These observations were obtained from the Radar Altimeter Database System (RADS) of the Netherlands Earth Observation Network (NEONET). This database provides processed sea surface height (SSH) anomaly measurements from the European Remote-Sensing (ERS), Geosat Follow on (GFO), TOPEX/Poseidon (T/P), and Jason satellite altimeters. The ERS and GFO satellites provide coverage over most of the state estimate domain, and the $\mathrm{T} / \mathrm{P}$ and Jason satellites provide coverage equatorward of $\sim 67^{0} S$. Along orbital track data are binned into the state estimate grid for each day of the simulation; no alteration or interpolation of the RADS data are performed. Each SSH anomaly observation provided by RADS is added to a mean 


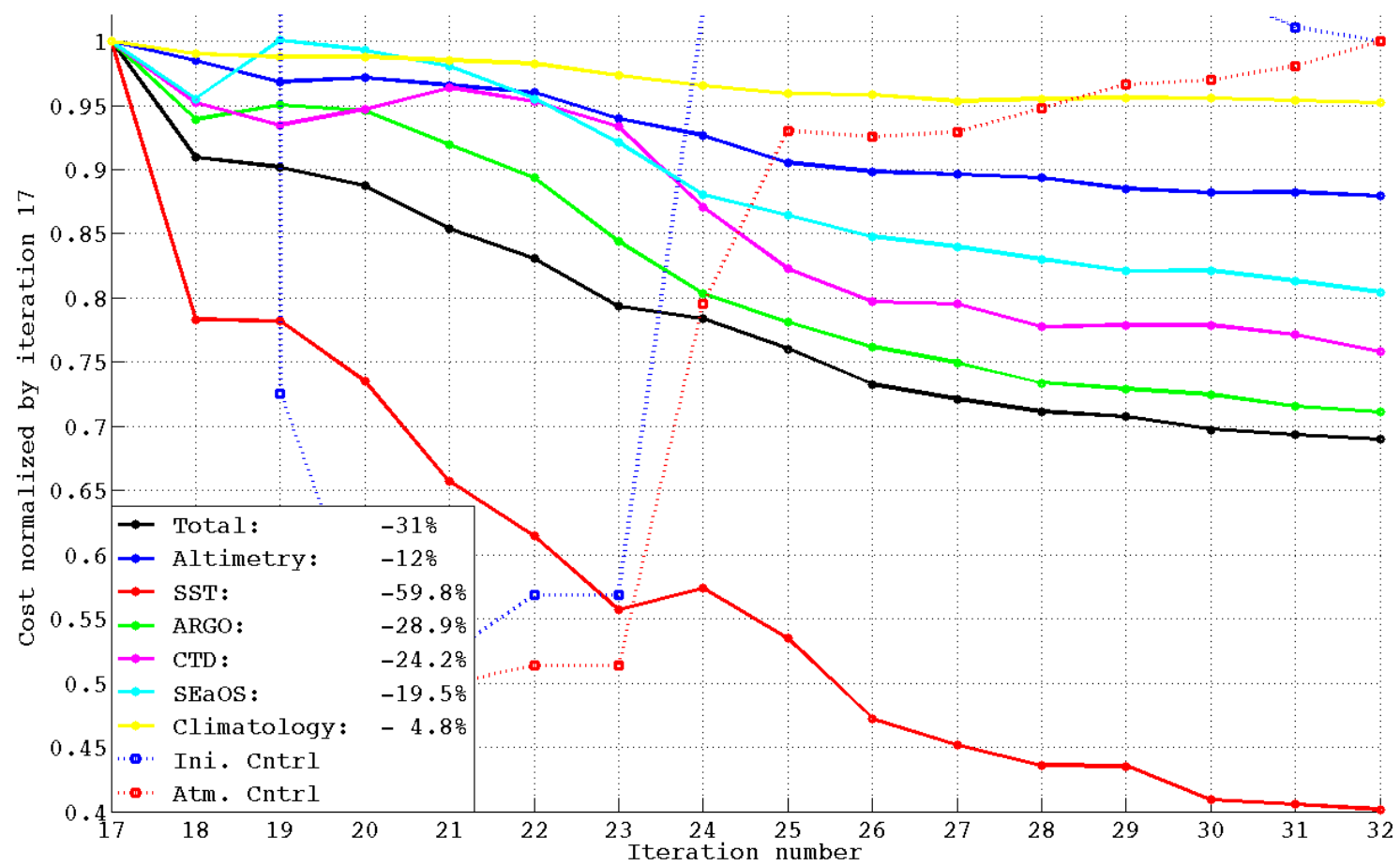

Figure 2-1: Cost terms normalized by their iteration 17 values (solid lines). Significant optimization occurred prior to iteration 17, however iteration 17 was the last time the cost function was altered and thus progress since this point is easily monitored and displayed. It is apparent that the cost continues to be reduced. The percentage of cost change since iteration 17 is shown in the legend. XBT in situ data have been included with the CTD cost. Note the costs associated with atmospheric state and initial condition control vector perturbations (dashed lines) increased as perturbations made to these fields brought them further from the state they were constrained to. They remain, however, acceptable (e.g. the atmospheric state is largely consistent with NCEP). All cost terms have been normalized by their iteration 32 values.

ocean dynamic topography product provided by the Gravity Recovery and Climate Experiment (GRACE) project to derive an absolute sea surface height observational data set. The model's sea surface height is averaged daily, the time and spatial mean offset is subtracted off, and the resulting field is compared to the observations. One is referred to the NEONET website (http://www.neonet.nl/) for more information regarding the altimetry anomaly data, and the GFZ German Research Centre for Geosciences website (http://www.gfz-potsdam.de/) for more information regarding the GRACE project mean dynamic ocean topography.

Prior to iteration 16 the data were used to constrain both the model's mean 
and time-varying component of SSH. The separation of these two components was made in order to distinguish errors associated with the geoid from those due to the time-evolving dynamics. The mean ocean dynamic topography data set, which is constructed relative to the Gravity Recovery and Climate Experiment (GRACE) project geoid, is processed and provided by the GFZ German Research Centre for Geosciences. This mean state is quite smooth spatially, and was made over a longer time period than the Southern Ocean state estimate. It is not clear how relevant this product is to the estimate's shorter time period and higher resolution mean. By using the altimetry observations as absolute measurements, this issue is avoided.

\subsubsection{Climatology}

The temperature and salinity model state monthly means are constrained to the monthly hydrographic climatology obtained from the NOAA World Ocean Atlas 2000 (Levitus et al., 2001; Conkright et al., 2002) from the surface to 300 meters. At depths below 300 meters the model is constrained to the temporally constant climatology of Gouretski and Koltermann (2004). These climatological constraints are given little weight owing to the fact that these products are quite smooth and that their consistency with modern observations is questionable. Nevertheless, they are important products because there are locations where they are able to provide the only constraint.

\subsubsection{In Situ Measurements}

The model is constrained to autonomous Argo float profile data, CTD (conductivity, temperature, and depth) and XBT (expendable bathythermograph) shipboard collected data, and data taken via instrument mounted seals. The XBT profiles come largely from D. Behringer (NCEP). The Argo float profiles are attained primarily from IFREMER (http://www.ifremer.fr/). The instrument mounted seal profiles are attained from the Southern Elephant Seals as Oceanographic Samplers (SEaOS) project (http://biology.st-andrews.ac.uk/seaos/). Observational profile values at each model 


\begin{tabular}{|l|l|r|}
\hline Instrument & Observation & \# for 2005-2006 \\
\hline ARGO & Salinity & $2,072,885$ \\
\hline CTD & Salinity & 30,731 \\
\hline SEAOS & Salinity & 162,993 \\
\hline ARGO & Temperature & $2,132,968$ \\
\hline CTD & Temperature & 30,793 \\
\hline SEAOS & Temperature & 212,661 \\
\hline XBT & Temperature & 47,097 \\
\hline
\end{tabular}

Table 2.1: In situ observations of the Southern Ocean taken in 2005 and 2006. Argo observations are primarily in and equatorward of the ACC. SEaOS observations are primarily poleward of the ACC.

depth level (when available) are used as constraints. These constraints total 2,266,609 for in situ salinity observations, and 2,423,519 for in situ temperature observations (see Table 2.1). A profiling package samples each model level at the nearest time step to each observation, and then interpolates to the observation location. There is minimal spatial and temporal model sampling error when calculating misfits to in situ data.

\subsubsection{Sea Surface Temperature}

Two different monthly mean sea surface temperature products are used to constrain the model. The first, distributed and processed by NCEP/Reynolds, determines sea surface temperature using data from the infrared channels of the Advanced Very High Resolution Radiometer (AVHHR). The other product, distributed and processed by Remote Sensing System (RSS), is obtained using microwave radiometer data (TMI, AMSRE). Both products are distributed at $1 / 4^{\circ}$ resolution, and are interpolated to the model grid for comparison. For more information on the the NCEP/Reynolds product see http://podaac.jpl.nasa.gov/sst/, and for more on the RSS product see http://www.ssmi.com/tmi/tmi_browse.html. 


\subsubsection{Sea Ice}

Observations of daily sea ice concentration have been attained from the National Snow and Ice Data Center (NSIDC). The data were collected by the Special Sensor Microwave Imager (SSM/I), and have been processed using the NASA Team algorithm. See http://nsidc.org/ for more information.

\subsubsection{Uncertainty}

Estimating error covariance matrices for the data and controls is a difficult and time consuming task of great importance. The state estimate is no better than the choice of these matrices as the weighting of the cost function determines the solution. If the error is underestimated, the model is constrained to noise; if the error is overestimated, useful information is discarded. Unfortunately, like the Southern Ocean's role in the global climate, there is a gap between significance and knowledge. Much work is still needed in determining the expected misfit when fitting modern ocean models to observations.

The weighting matrices used in this work are ever evolving; if a constraint appears to be too loose or too tight, the uncertainty estimate is evaluated and, if thought appropriate, altered. The current weighting matrices used in the state estimation, which include only the diagonal terms of the error covariance matrix, are adopted from the ECCO-GODAE $1^{\circ}$ resolution global state estimate. One is referred to this work (see for example Lu et al. (2002); Wunsch and Heimbach (2005); Forget and Wunsch (2007)) for an accounting of the fields. A description of the uncertainty estimates is also given in Mazloff (2006). A notable improvement since the description given in Mazloff (2006) is that the sea surface temperature observational uncertainty estimate is now spatially varying. 

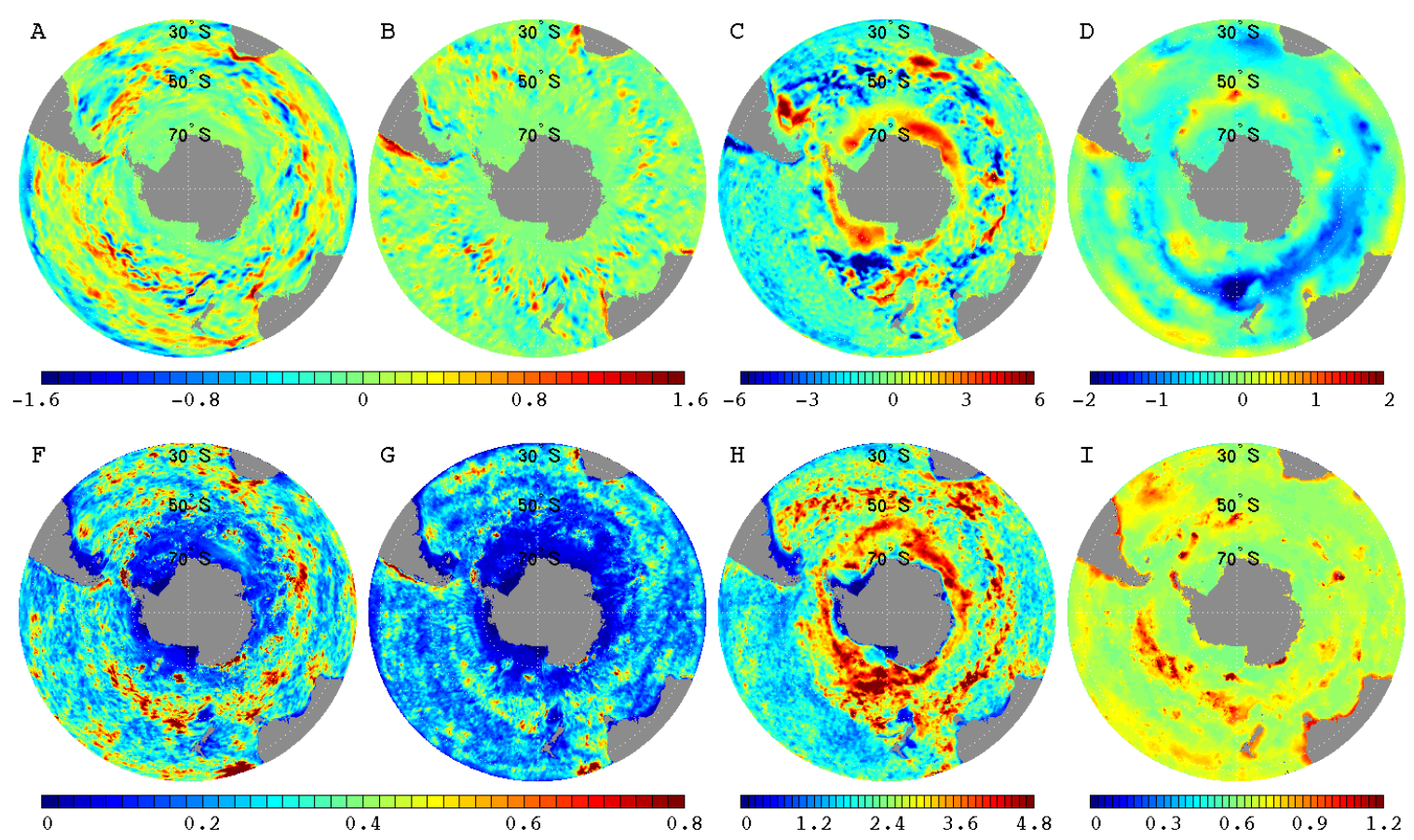

Figure 2-2: Time-mean perturbations to the NCEP atmospheric state and their standard deviations (see Section 1.3.1) for iteration 32: the zonal wind speed $[\mathrm{m} / \mathrm{s}](\mathrm{A}$ : mean and F: standard deviation), the meridional wind speed $[\mathrm{m} / \mathrm{s}]$ (B: mean and G: standard deviation), the atmospheric temperature $\left[{ }^{0} \mathrm{C}\right](\mathrm{C}$ : mean and $\mathrm{H}$ : standard deviation), and the precipitation [mm/day] (D: mean and I: standard deviation). Perturbations to the short wave radiative flux and the specific humidity are not shown. Adjustments to the model inputs are responsible for bringing the model state closer to consistency with the observed ocean.

\subsection{The Control Vector}

The first guess atmospheric state provided by the NCEP/NCAR Reanalysis Project (Kalnay and coauthors, 1996) has been adjusted in order to bring the model into consistency with the observations described in Section (2.1). The mean and standard deviations of the iteration 32 control vectors for the wind fields, the atmospheric temperature, and the rate of precipitation are shown in Figure (2-2). The significant wind field changes are of relatively small spatial scales, while changes to the other components are basin scale. Though both the spatial and temporal variability in the wind has been significantly increased in the ACC region, it is interesting to note that the mean Southern Ocean kinetic energy in the state estimate has dropped approxi- 
mately $5 \%$ from the initial run to iteration 32 . Whether the state estimates kinetic energy decrease occurred primarily in the mean or the transient flow components has yet to be determined.

The optimization has cooled the atmosphere equatorward of the ACC, and warmed it over most of the polar gyres (panel C). There are exceptions to this pattern, however; for example the atmospheric temperature over the northwestern Ross Sea has been made significantly cooler. Atmospheric temperature perturbations are significantly variable in time (i.e. the standard deviation, panel $\mathrm{H}$, is the same order as the mean). Changes to the short wave radiation (not shown) reflect this same pattern of cooling and heating, and of increased variance near the pole. It is interesting, however, that the perturbations to the initial temperature show an opposite pattern, with cooling in the polar gyres and warming in the subtropical gyres (not shown). Of course one should not expect changes at the surface to reflect changes in the ocean interior.

The optimization has, for the most part, increased rainfall in the subtropical and polar gyres, and decreased rainfall over the ACC region (Panel D). The variability of the precipitation adjustments is greater in the ACC and coastal regions (panel I). Specific humidity perturbations are of a similar pattern to that shown for the precipitation field (not shown). As with the perturbations to the temperature initial condition, it is interesting to note that perturbations to the salinity initial condition also have a pattern in opposition to the influence of surface flux changes. The initial salinity has been decreased in the subtropical gyres and increased in the polar gyres.

To reduce the cost there are several inputs that the optimization may perturb, and many of these have overlapping effects. For example, to heat the ocean the adjoint method could either increase shortwave radiation into the ocean, or increase the atmospheric temperature. However changing the atmospheric temperature may change the atmospheres stability, and thus the wind stress value through the drag coefficient. Similarly, increasing atmospheric specific humidity will decrease evaporation, and thus one may freshen the ocean by either increasing specific humidity or precipitation. Only increasing specific humidity, however, can also increase wind 
stress as it increases the atmospheres density. Each control input is also weighted differently. For these reasons it is not obvious exactly why each specific control has been perturbed in this optimization.

The changes made in the Southern Ocean state estimate are remarkably similar to those shown in the published $1^{0}$ ECCO global state estimate solution of Stammer et al. (2004). This is true for all the mean fields (compare panel A and B to their Figure $9 \mathrm{C}$ and D, and compare panel C and D to their Figure $3 \mathrm{C}$ and D). In the work of Stammer et al. (2004), it was concluded that some regional small-scale wind stress adjustments reflected the ocean model's failure to resolve intense boundary currents. That these same corrections show up in the much higher resolution Southern Ocean state estimate suggests that perhaps it is more than just model error being corrected. It is possible that the NCEP fields are poor near continents, and need corrections due to their own inadequacies. That the mean changes necessary to improve the model state are, to a certain degree, ocean model resolution independent is a reassuring result. It gives promise to improving first guess atmospheric state fields for future state estimates, making the prospect of eddy resolving state estimates better. Furthermore, finding consistency in the perturbations with a solution that has been well evaluated and compared to other NCEP error inferences (e.g. Large and Yeager (2008)) gives some credibility to the Southern Ocean state estimates optimized fluxes.

\subsection{Model-Observation Fit}

The state estimate is a solution to a high-resolution state-of-the-art numerical model. Much of what is known about the Southern Ocean dynamics comes from evaluation of similar models (e.g. Marshall et al. (1993) and Saunders and Thompson (1993) with FRAM, and Lee et al. (2007) with OCCAM). In this work, the initial model solution was determined with what was arguably the most realistic boundary and initial conditions available. The state it produced, however, was inconsistent with observations in many respects. These inconsistencies were significantly reduced via optimization using the adjoint method. A detailed comparison of the state estimate solution to 
observations has been made. Having such an evaluation is a major advantage over past model analyses, as inferences drawn from this solution may, in some sense, be tested.

The goal of the state estimate is to bring the model state into consistency with observations. If each observation were independent, which is not the case for some observations (e.g. the climatology, due to its processing), and had a Gaussian error distribution, then consistency would be defined such that the mean of the misfit would approach zero, and the variance of the normalized misfit (misfit magnitude divided by uncertainty) would approach one. The cost, which is the normalized misfit squared,

should then have a $\chi_{1}^{2}$ distribution. In a $\chi_{1}^{2}$ distribution it is expected that $\sim 68 \%$ of the cost values will be less than one. A small percentage, however, may have cost values far greater than one.

The normalized cost values for iteration 17 to 32 are greater than one (Figure 2-3). As these cost values are normalized by their prescribed uncertainties, their consistency is as well determined as this uncertainty. A comparison, and subsequent evaluation of the state estimate to observations is therefore incomplete without a rigorous discussion of the uncertainties in both the model and ocean measurements. Such a discussion, however, is beyond the scope of this thesis. In this section the focus will be on the unweighted misfit of the state estimate to the observations. It is the hope that although this diminishes the quality of the evaluation, omitting weighted misfit squared (cost) plots will make the discussion more accessible to the reader.

\subsubsection{In Situ Measurements}

\section{Temperature}

The misfit of the model state to the in situ ocean temperature observations is quite small over most of the Southern Ocean. There is, however, a misfit dependence on depth and latitude (Panel B of Figure 2-4). On average, the model temperatures just below the mixed layer are $\sim 0.3^{0} \mathrm{C}$ cooler than the observations equatorward of the Antarctic Circumpolar Current fronts, and $\sim 0.8^{0} \mathrm{C}$ warmer poleward of these 


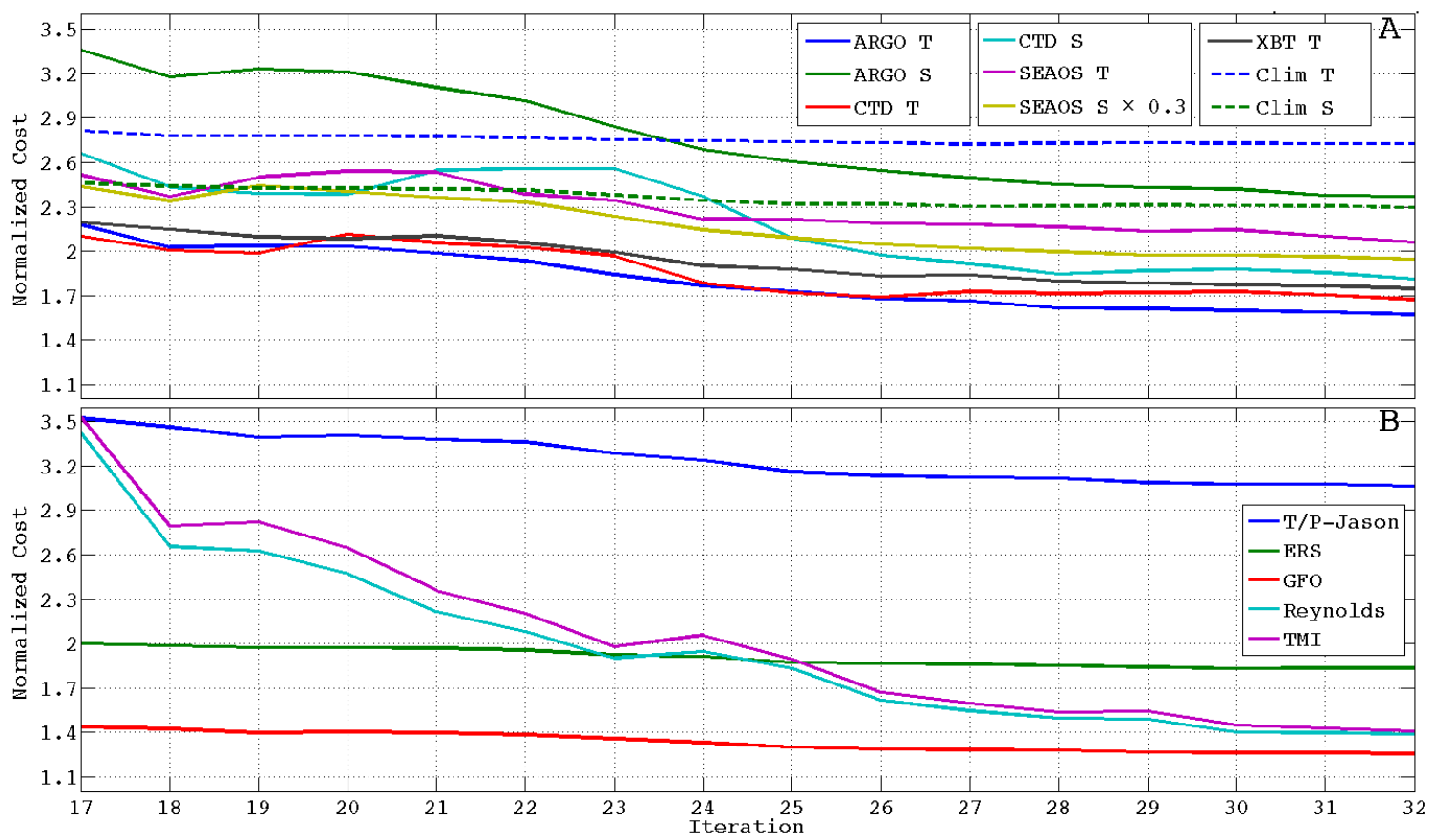

Figure 2-3: Normalized cost terms for iteration 17 to iteration 32. The cost terms for each data set are multiplied by a different constant before summed in the full cost function; the total cost is not a simple sum of the values plotted. The SEaOS salinity normalized cost is quite large and has thus been multiplied by 0.3 in the Panel A plot. It is possible that the prescribed uncertainty for this data set (and all the others) is too small.

fronts. The sign and magnitude of this misfit switches below $\sim 150$ meters. Below $\sim 500$ meters the misfit magnitude diminishes. It is important to note that the zonal average misfit plotted in Panel B is not a true zonal average. Panel A shows that data coverage in the polar gyres is lacking, and the misfit pattern in the high latitudes of Panel $\mathrm{B}$ is dominated by a few regions. Panel A shows that most of the errors are associated with misfits of front strength or location. This is to be expected as this is where the temperature gradients are the largest, and thus where misfits are expected to have the largest magnitude.

\section{Salinity}

The patterns of the misfits of the model state to the ocean in situ salinity (Figure 2-5) are somewhat complementary to the temperature misfits, where the estimate is too cool (warm), it is often too fresh (saline). This suggests the model may be closer 


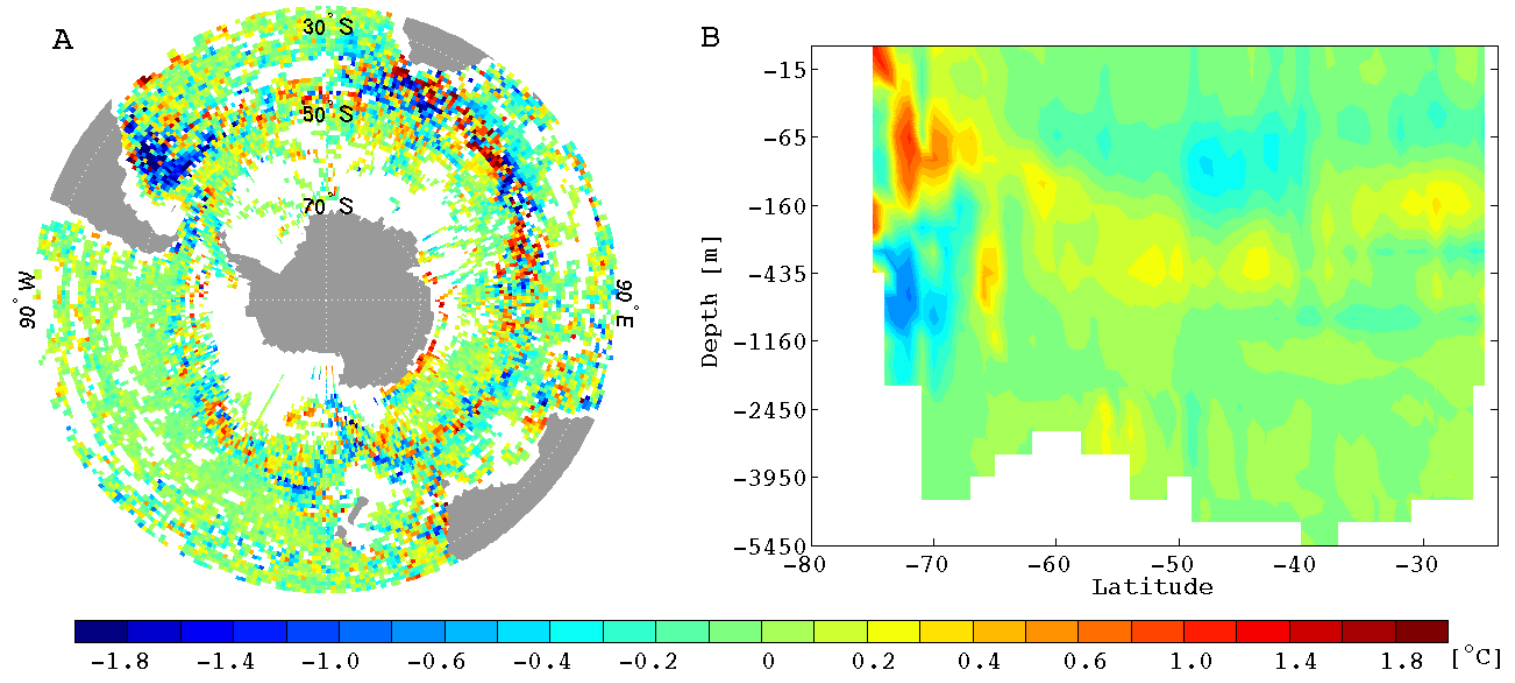

Figure 2-4: The Southern Ocean state estimate temperature $\left[{ }^{\circ} \mathrm{C}\right]$ minus in situ observations (positive values denote the model state is warmer than the observations). The misfit has been binned into monthly averages on a $1^{\circ}$ grid with 23 vertical levels. Panel A is vertically and temporally averaged and Panel B is longitudinally and temporally averaged. Note that the color axis is nonlinear and that in Panel B the vertical scale is stretched.. The apparent larger misfit at high latitudes in Panel B may be dominated by a few observations, as data are more sparse near Antarctica.

to the truth in density, rather than in temperature or salinity alone. As with the temperature misfit, there is a clear dependence on depth and latitude. The estimate is too saline from Antarctica through the locations where Antarctic Intermediate Waters are found (Panel B ). The surface waters north of the Subantarctic Front are slightly fresher than observed. Though again, one must realize that observations become sparse near the pole and thus the zonal average becomes dominated by a few regions. The largest salinity misfits are associated with the strong fronts of the Antarctic Circumpolar Current (Panel A).

\section{Water Masses}

The water classes of the Southern Ocean, which are discussed in Section (3.1.1), are reproduced in the model (Figure 2-6). The observed profiles are somewhat tighter in temperature and salinity (TS) space than is the state estimate, suggesting that the estimate may be overemphasizing the degree of diffusion and mixing. While the fit 


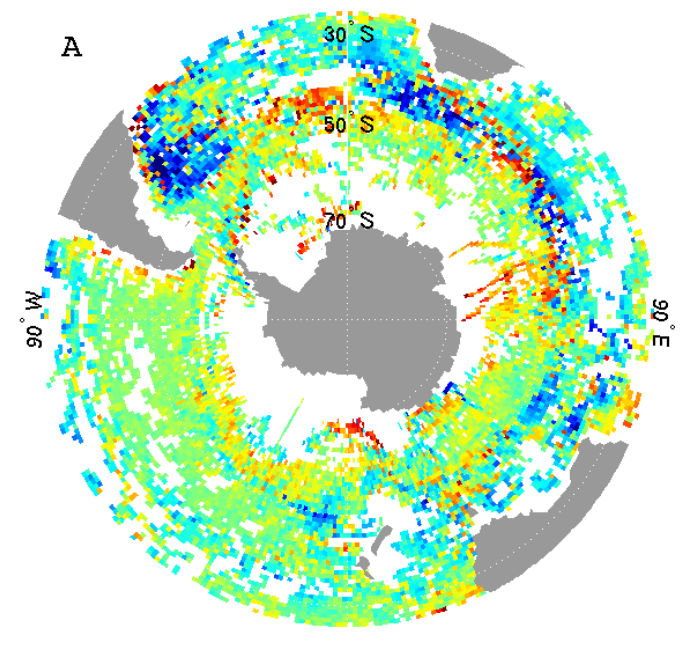

\begin{tabular}{lllll|l|l|l|l|l|l|l|}
\hline-0.38 & -0.29 & -0.21 & -0.14 & -0.09 & -0.05 & -0.02
\end{tabular}

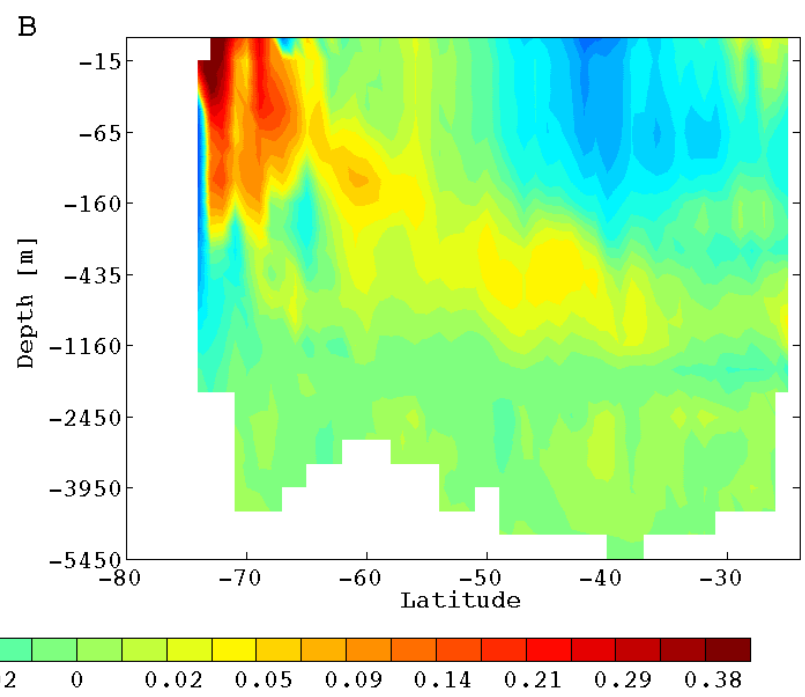

Figure 2-5: Southern Ocean state estimate salinity minus in situ observations (positive values denote the model state is more saline than the observations). The misfit has been binned into monthly averages on a $1^{\circ}$ grid with 23 vertical levels. Panel A is vertically and temporally averaged and Panel B is longitudinally and temporally averaged. Note that the color axis is nonlinear and that in Panel B the vertical scale is stretched. The apparent larger misfit at high latitudes in Panel B may be dominated by a few observations, as data are more sparse near Antarctica.

isn't perfect, there are no large discrepancies; the state estimate has no missing water masses in these meridional sections.

\subsubsection{Altimetry}

The combined model-data uncertainty assigned to the $\mathrm{T} / \mathrm{P}$ and Jason altimetric observations is approximately $10 \mathrm{~cm}$ (see Section 2.1.1 for a discussion of the satellite altimeters used). There are high levels of eddy activity in the Antarctic Circumpolar Current region, and as a result the mean misfit in this area reaches values of approximately $30 \mathrm{~cm}$ (Panel A of Figure 2-7). Outside of the ACC region the time average misfit is, for the most part, less than $10 \mathrm{~cm}$ and thus acceptable. The same conclusion can be drawn for the ERS and GFO altimetric observations (Panels B and C of Figure 2-7) when one considers that the uncertainties in these observations are slightly larger than for T/P and Jason altimeters. There is a noticeable bias of the state estimate's mean sea surface height; the SSH is lower than observed north of the ACC and higher 

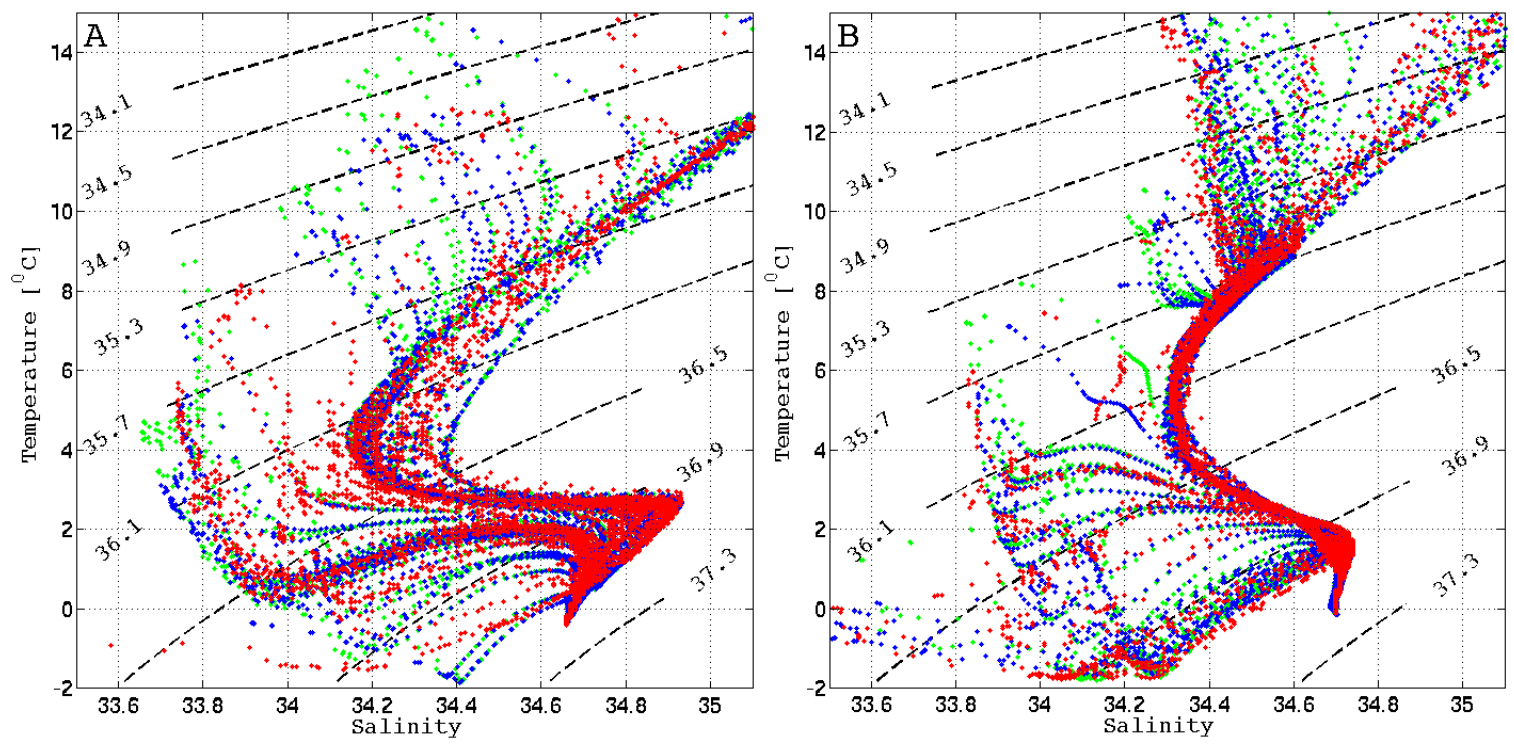

Figure 2-6: Temperature and salinity (TS) space plot for a CTD synoptic sections taken in January and February of 2005 from $60^{\circ} \mathrm{S}$ to $25^{\circ} \mathrm{S}$ at $30 \mathrm{~W}$ in the South Atlantic Ocean (Panel A) and from $71^{\circ} \mathrm{S}$ to $25.5^{\circ} \mathrm{S}$ at $150 \mathrm{~W}$ in the South Pacific Ocean (Panel B). The iteration 0 values are plotted in green, over that the iteration 26 state estimate values are plotted in blue, and over both the observations are plotted in red. The state estimate water masses are slightly more spread in TS space, suggesting an overestimate of diffusion and mixing. There are more outliers, especially in the more buoyant water classes, in the iteration 0 solution. Potential density contours (referenced to $2000 \mathrm{db}$ ) are plotted.
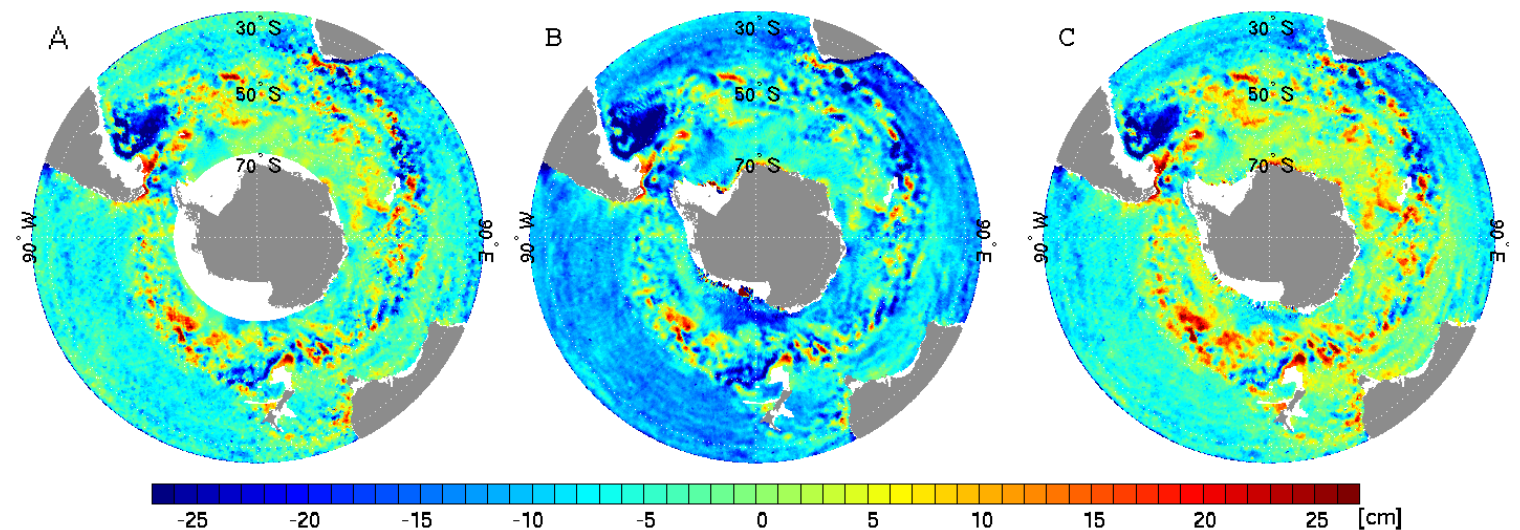

Figure 2-7: Time mean state estimate sea surface height misfit to observations in $\mathrm{cm}$. The observations are from the GFZ dynamic ocean topography combined with anomaly observations from A) combined T/P and Jason, B) ERS, C) GFO. See subsection (2.1.1) for more information regarding the observations.

than observed south of the ACC. (For the ERS altimeter the estimated SSH is lower than observed everywhere outside the ACC latitudes.) Though the magnitude of this 
bias is small, it is systematic and will likely have to be corrected through further iterations.

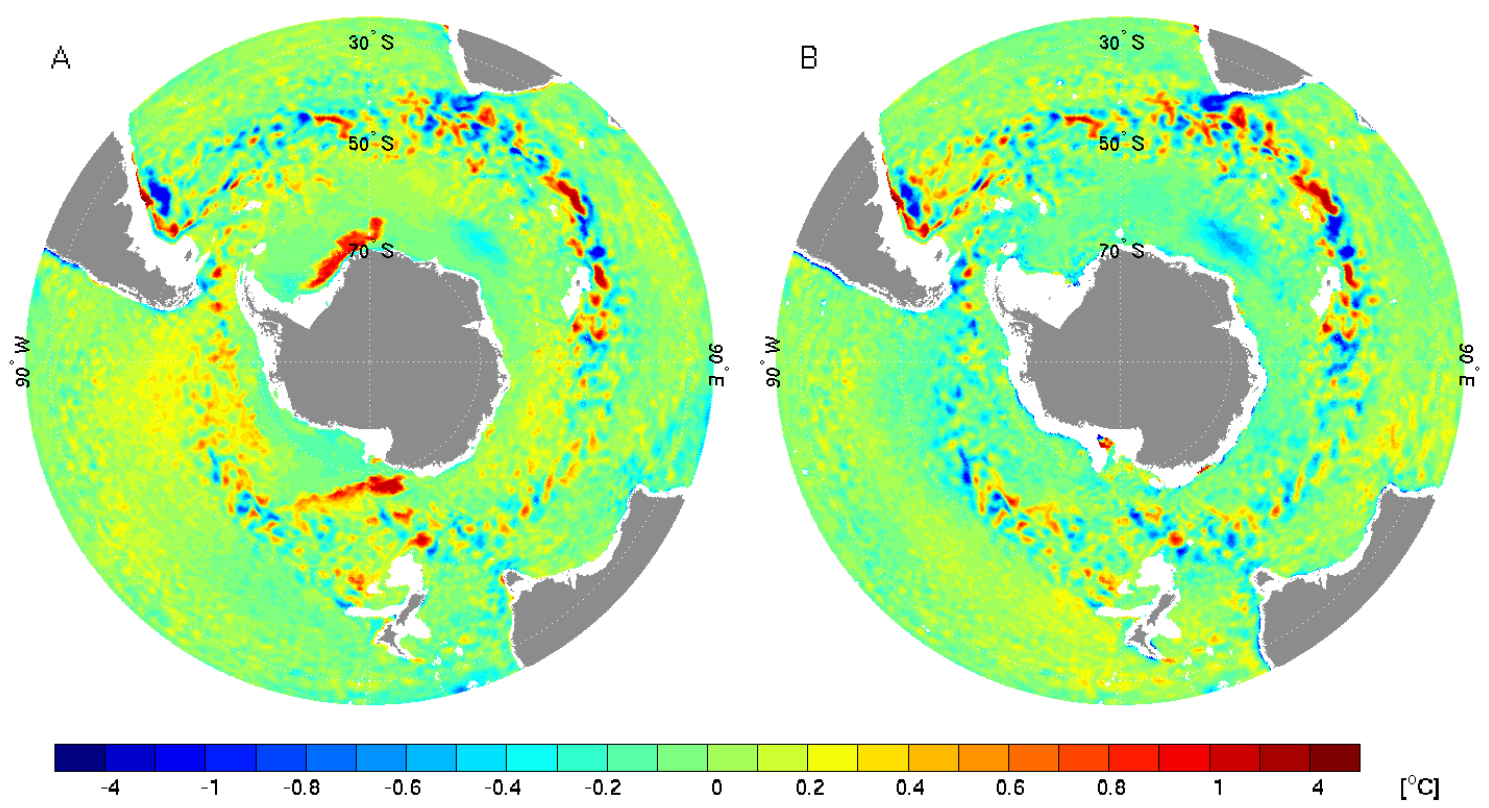

Figure 2-8: Time average misfit: state estimate sea surface temperature (SST) observed SST from infrared (Panel A) and microwave (Panel B) radiometer data. For infrared (microwave), 95\% (94\%) of the area has a misfit of less than $1^{0} \mathrm{C}$, and $61 \%(60 \%)$ is less than $0.3^{0} \mathrm{C}$. Note that the color axis is nonlinear.

\subsubsection{Sea Surface Temperature}

There are some significant differences in the mean misfit of the state estimate to the two constraining sea surface temperature (SST) radiometer data products (Figure 28), which denotes differences between the two products. The magnitude of the misfit to both products is quite small $\left(60 \%\right.$ of the area has a misfit of less than $0.3{ }^{0} \mathrm{C}$ ), and most differences are within the expected uncertainty. There are, however, some notable biases. The estimate is too warm in the subtropics. In the ACC latitudes there are blotchy misfits likely due to discrepancies in the frontal locations. There are two striking regions in the polar gyres where the estimate is order $1^{0} \mathrm{C}$ warmer than the infrared observational product. Winter time polynyas are produced in these 
regions by the state estimate, but were not observed in either 2005 or 2006 . The polynyas may be a result of the relatively warm surface waters (Martinson et al., 1981; Motoi et al., 1987).

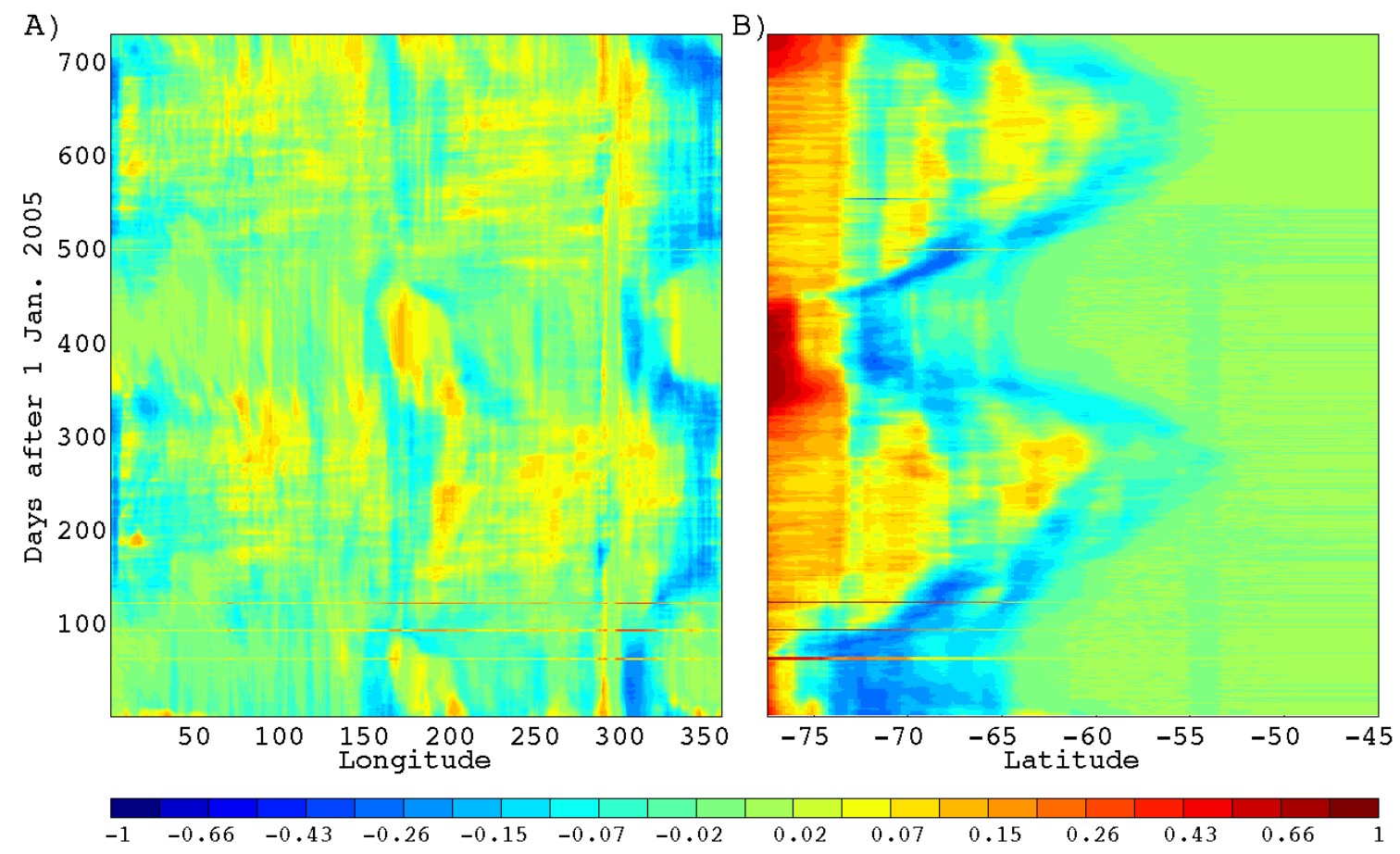

Figure 2-9: Sea ice concentration misfit (estimated minus minus observed). The concentration ranges from 0 to 1 , such that the maximum misfit, which has magnitude $1(-1)$, occurs if a model (observed) cell is completely ice covered and there is no observed (modeled) ice. The misfit has been averaged latitudinally in the left panel, and averaged longitudinally in the right panel. Note that the color axis is nonlinear.

\subsubsection{Sea Ice}

The state estimate has, in general, a greater sea-ice concentration than is observed in the South Indian and South Pacific regions from longitudes $30^{\circ} \mathrm{E}$ to $300^{\circ} \mathrm{E}$, and has less sea-ice than is observed in the South Atlantic region from $300^{\circ} \mathrm{E}$ to $30^{\circ} \mathrm{E}$ (Panel A of Figure 2-9). The ice deficiency in the Weddell sea may be attributed to a polynya that forms in the estimate. The Ross Sea is the primary region that reaches latitudes poleward of $\sim 75^{\circ} \mathrm{S}$, and there is an excess of ice in this region in Austral summer (Panel B). This excess may be caused by the absence of sea-ice dynamics in the estimate, and thus the lack of a flushing effect in the polar gyres. The seasonal 


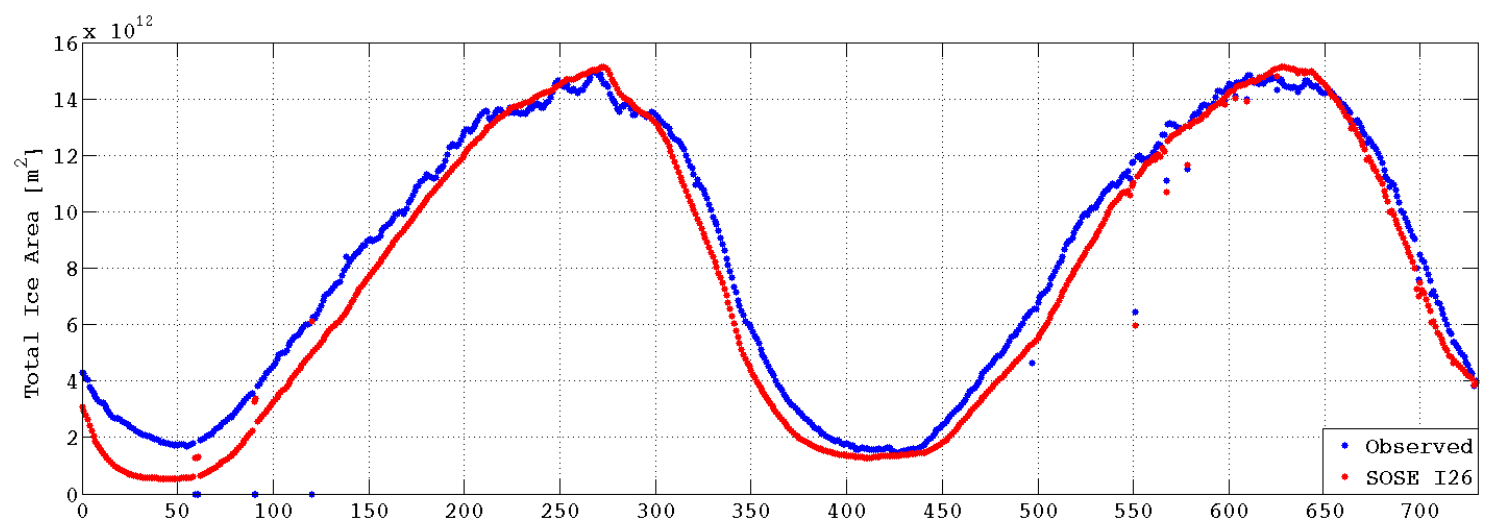

Figure 2-10: Total sea ice area in the Southern Ocean: Observed from NSIDC (blue) and estimate (red). The seasonal change is slightly stronger and more rapid in the estimate than in the observations. Neglecting the first couple months, the average difference in the 2 curves is approximately $11 \%$.

cycle of sea ice growth and retreat is, for the most part, captured (Figure 2-10). The integrated area peak value in the state estimate is close to what is observed. The state estimate seems to better reproduce the observed area in 2006.

\subsubsection{Summary}

Though the state estimate is still converging, the solution is useful for analysis. To show this, the cost (weighted misfit squared) of the solution to in situ observations is evaluated in Figure (2-11.) The cost magnitude is compared with that calculated from the 2001 World Ocean Atlas, WOA01, climatological product (Stephens et al., 2001) and a coarse resolution state estimate (Forget, 2008). As explained above, a cost greater than one implies the model state is outside the uncertainty bounds prescribed. This chapter has avoided discussing the prescribed uncertainty as this is a complicated subject involving consideration of intrinsic model errors, observational measurement errors, and errors associated with the models representation of the ocean state (Forget and Wunsch, 2007; Wunsch and Heimbach, 2007). One can, however, gain an idea of the adequacy of the Southern Ocean state estimate regardless of weighting by evaluating the relative costs. For each observational data set, at almost all latitudes, both state estimates are more consistent with the observations than is the climatology. Much of what is known about the ocean comes from studies 

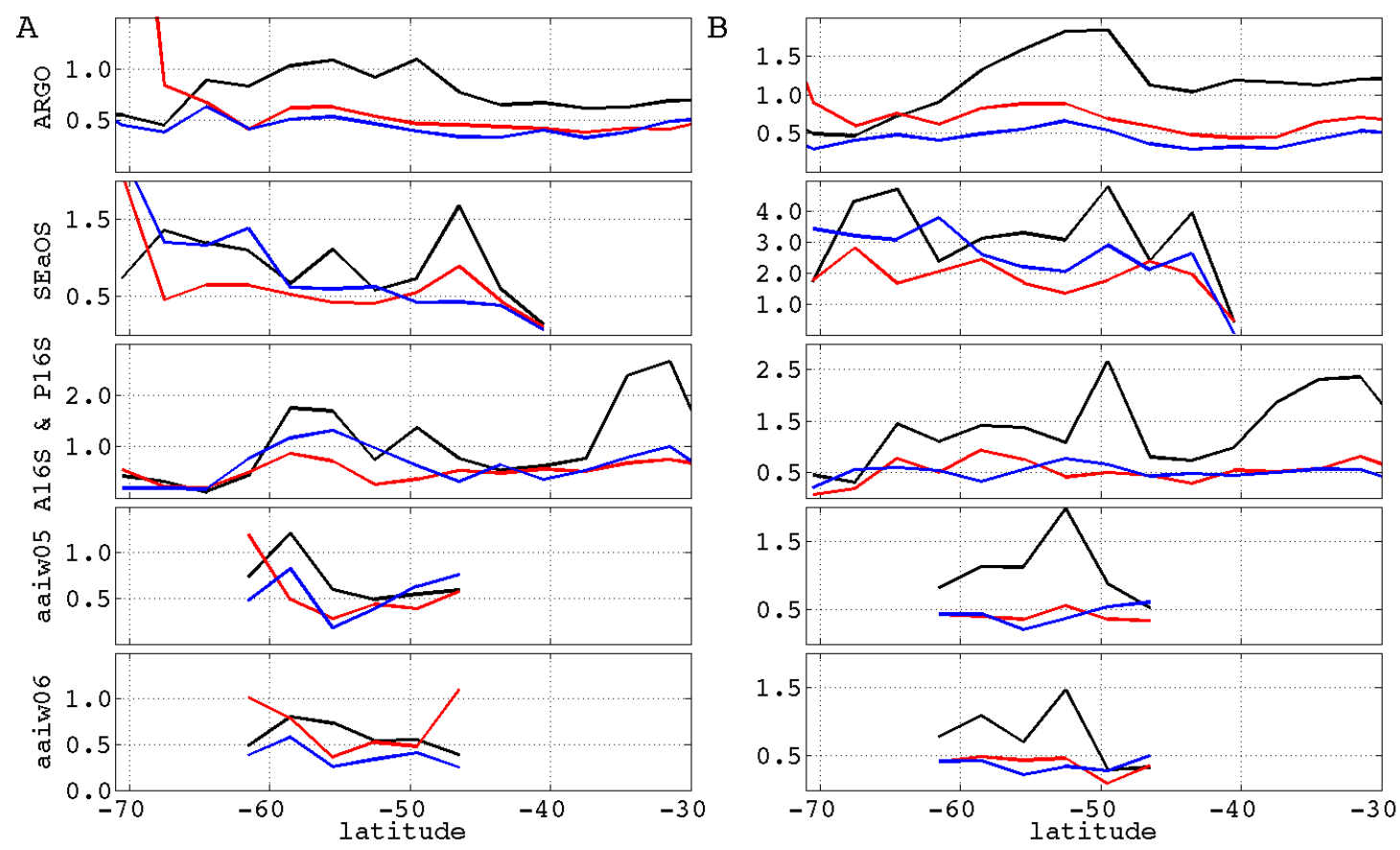

Figure 2-11: The median cost value (weighted misfit squared) for the World Ocean Atlas 2001 (black line), a coarse resolution state estimate (blue line; Forget, 2008), and the Southern Ocean state estimate (red line) with respect to latitudinal bins of various in situ temperature (Panel A) and salinity (Panel B) observations. From top to bottom, the in situ observational data sets are from the Argo project, the SEaOS project, combined A16 and P16 hydrographic sections, and two hydrographic sections from the Antarctic Intermediate Water Formation in the Southeast Pacific project (aaiw 2005 and aaiw2006; see http://tryfan.ucsd.edu/aaiw/aaiw.htm for more information). For the most part, the Southern Ocean state estimate exhibits lower cost than the World Ocean Atlas 2001, implying the state estimate is more consistent with this large influx of modern data.

employing climatological products such as the WOA01. The Southern Ocean state estimate is, however, a better representation of the modern ocean than the WOA01, and this validates its usefulness for analysis.

As can be seen in the misfit and cost plots in this chapter, there is room to improve the state estimate. This involves gathering more data, improving the model set-up, and continuing to iterate. To determine when the solution has converged to an acceptable misfit with the observations will involve analyzing and refining the uncertainties. One will need to determine what data are good, lacking, and problematic. Calculating appropriate uncertainties for use in a coarse resolution state estimate is 
a serious outstanding problem (Forget and Wunsch, 2007). Calculating appropriate uncertainties for use in an eddy permitting state estimate, like the one at hand, becomes even more challenging. Future work must determine the best way to constrain eddy resolving state estimates to observations. This includes determining the cost function structure. For example, is it desirable and feasible to reproduce the ocean's eddy field, or is it wiser to reproduce only the eddy statistics. It is likely the cost function should be designed to capture the eddy statistics, and work to determine how this should be done is being carried out (e.g. Köhl and Willebrand (2002)). Weights for eddy-permitting state estimates that reflect the presence of eddies in both the observations and the model will have to be determined. Cost function terms designed to capture eddy statistics will need to be weighted as well. Only then will it be possible to fully determine the acceptability of a solution. It is important to note that though the solution is not completely consistent with the observed ocean, it is, like all the ECCO state estimates, dynamically self consistent. The conclusion drawn in this chapter is simply that a non-converged state estimate has been produced that is good enough for analysis. Indeed, the author knows of no other eddy permitting estimate of the modern state of the Southern Ocean that is more consistent with the observations. 


\section{Chapter 3}

\section{Analysis of a Southern Ocean State Estimate}

\subsection{Introduction}

This chapter describes the circulation and transport of the Southern Ocean state estimate. An introduction to the Southern Ocean fronts and water classes is presented in order to facilitate the transport discussion. Section 3.2 describes the estimated horizontal freshwater, heat, and volume transport. This includes a discussion of the Ekman transport and the meridional overturning volume transport. The chapter concludes with a brief section regarding the rate of work done by the wind on the Southern Ocean. The reader should remember that the estimate being analyzed is not yet fully consistent with observations. Chapter 2 described the "goodness" of the state estimate analyzed here. That "goodness" information can be used to gauge the accuracy of the inferences made in this thesis. Future work should determine a method for calculating accurate error bounds on the Southern Ocean circulation based on model-observation misfit. For now, only the analysis is presented and no further attempt to gauge uncertainty is made.

To aid in the discussion of the fronts and water masses of the Southern Ocean two year mean salinity and temperature fields along the $30^{\circ} \mathrm{W}$ South Atlantic meridional section are presented (Figure 3-1). A useful northern boundary for the Southern 

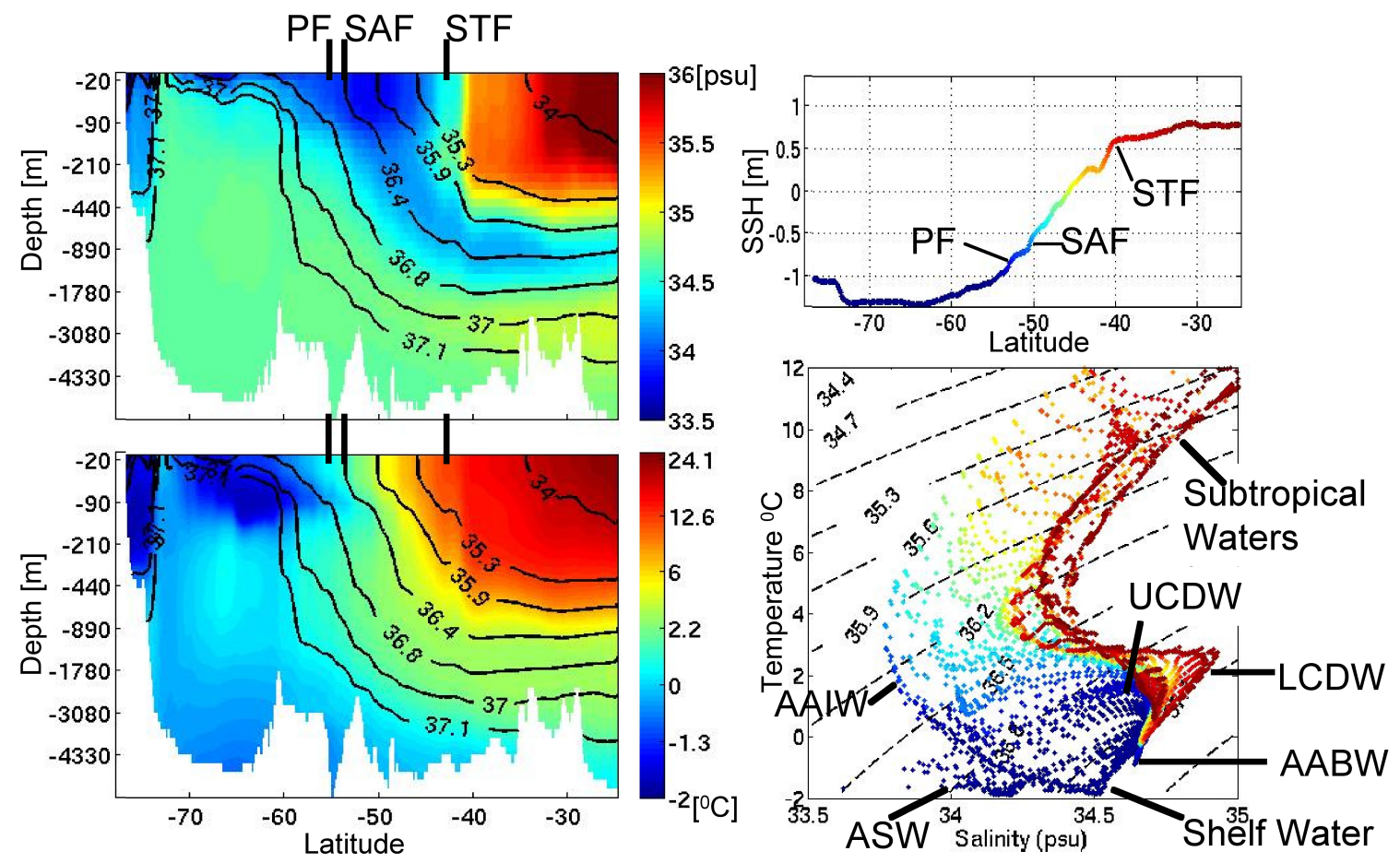

Figure 3-1: A meridional section from the state estimate in the Atlantic sector of the Southern Ocean $\left(30^{0} \mathrm{~W}\right)$. The two year time mean salinity (top left) and potential temperature referenced to the surface $\left[{ }^{0} \mathrm{C}\right]$ (bottom left) are plotted with potential density referenced to $2000 \mathrm{~m}$ contours overlaid. The top right plot shows the time mean sea surface height for this line. The color axis scales with the sea surface height (i.e. movement along the color-scale changes at a rate proportional to the sea surface height change). A temperature vs salinity (TS) diagram for the section is shown in the bottom right panel. Colors shown in this plot denote that the water mass plotted is found at the latitude corresponding to the colors used in the sea surface height plot. See Section 3.1.1 for water mass acronyms pointed out in the TS diagram. One may wish to compare this time-mean TS diagram with the synoptic sections plotted in Figure (2-6).

Ocean is the Subtropical Front (STF), as this is the southern boundary of the subtropical gyres of the South Atlantic, Indian, and South Pacific Oceans. Poleward of the STF is the Subantarctic Front (SAF), which is the northernmost front of the Antarctic Circumpolar Current. The SAF can be found between its southernmost extent in the Drake Passage at approximately $58^{\circ} \mathrm{S}$ and its northernmost extent in the western South Atlantic at approximately $45^{0} \mathrm{~S}$. To the south of the SAF, within the ACC system, one finds the Polar Front (PF). This front location varies from approximately $50^{\circ} \mathrm{S}$ in the South Atlantic and South Indian Oceans to approximately 
$60^{0} \mathrm{~S}$ in the South Pacific Oceans. The PF's southernmost extent at latitude $\sim 63^{0} \mathrm{~S}$, occurs just west of Drake Passage. The most poleward front of the ACC region is known as the Southern ACC Front. The proxies that allow one to recognize these fronts are not simple, as they often change depending on region. For this reason a description of these proxies is omitted, and one is instead referred to Pickard et al. (2008). However, in general, the SAF is characterized by the southern bound of the surface salinity minimum. The PF is generally characterized by the northern bound of the near surface temperature minimum.

Poleward of the Southern ACC front is the Subpolar Zone (also sometimes called the Antarctic Zone), which is characterized by westward winds. In the Southern Ocean state estimate produced, the time-averaged net poleward Ekman transport associated with these winds is negligible (see Figure 3-8). There is, however, a significant upwelling driven by the strong wind curl in the region. This upwelling drives the cyclonic Ross and Weddell Polar Gyres. A coastal current traveling westward along the majority of the Antarctic continent is also a prominent feature of this weakly stratified region.

Buoyancy forcing determines some of the Southern Ocean's hydrographic features. The primary feature is a significant salinity minimum found north of the Subantarctic Front, which results from a relatively high precipitation band centered around $50^{0} \mathrm{~S}$. Wind forcing and cooling then combine to subduct these Antarctic Intermediate Waters and drive them equatorward where they ventilate a substantial fraction of the world's oceans. Poleward of the $\sim 63^{0} \mathrm{~S}$ buoyancy forcing is dominated by ice dynamics; wintertime ice formation results in brine rejection, while summertime ice melt results in freshwater input. North of $\sim 63^{0} \mathrm{~S}$ buoyancy forcing is dominated by oceanic air-sea heat exchange (Cerovecki et al., 2008).

\subsubsection{Water Masses}

Buoyancy and wind forcing combine to facilitate a great deal of water mass conversion and formation. An introduction to the major Southern Ocean water classes is given in this section in order to facilitate a description of the Southern Ocean overturning. 
These waters are the Antarctic Surface Water (AASW), Subantarctic Mode Water (SAMW), Antarctic Intermediate Water (AAIW), Upper and Lower Circumpolar Deep Water (UCDW and LCDW), and Antarctic Bottom Water (AABW). For a more complete accounting of the Southern Ocean water classes see Pickard et al. (2008).

The polar waters found between Antarctica and the ACC's Polar Front have temperatures ranging from $-1.9^{0} \mathrm{C}$ to $2.5^{0} \mathrm{C}$. Typically the warmer waters are at intermediate depths and stable stratification is achieved through salinity gradients; AASW is much fresher than LCDW and AABW. LCDW is largely composed of North Atlantic Deep Water (NADW), and thus its primary source is inflow from the South Atlantic. This relatively salty LCDW mixes with near freezing Antarctic Shelf Water to form the densest waters found in the world oceans. This dense water mass, known as AABW, flows equatorward out of the Polar Oceans. AABW is a key ingredient in the world's abyssal waters.

Late winter convection generates SAMW, making this water class characterized by low potential vorticity and high oxygen. AAIW, which is formed from the densest SAMW, is denoted as the near surface to mid-depth salinity minimum water found between the Subantarctic and Subtropical Fronts. Nutrient rich UCDW flows into the Southern Ocean above the saltier LCDW. UCDW mixes with, and transforms, the AAIW. The AAIW buoyancy loss resulting from this mixing, combined with surface cooling and Ekman pumping, forces subduction at the Subtropical Front. As a result, cold and fresh AAIW ventilates the thermoclines of the southern hemisphere subtropical gyres. A surface Ekman driven inflow of Subtropical Waters also subducts at the Subtropical Front to join the AAIW outflow. Mixing of these two water masses along their equatorward flow is evident in the TS diagram of Figure (3-1). (Orsi et al., 1995; Rintoul et al., 2001; Sloyan and Rintoul, 2001; Tomczak and Liefrink, 2005; Pickard et al., 2008) 


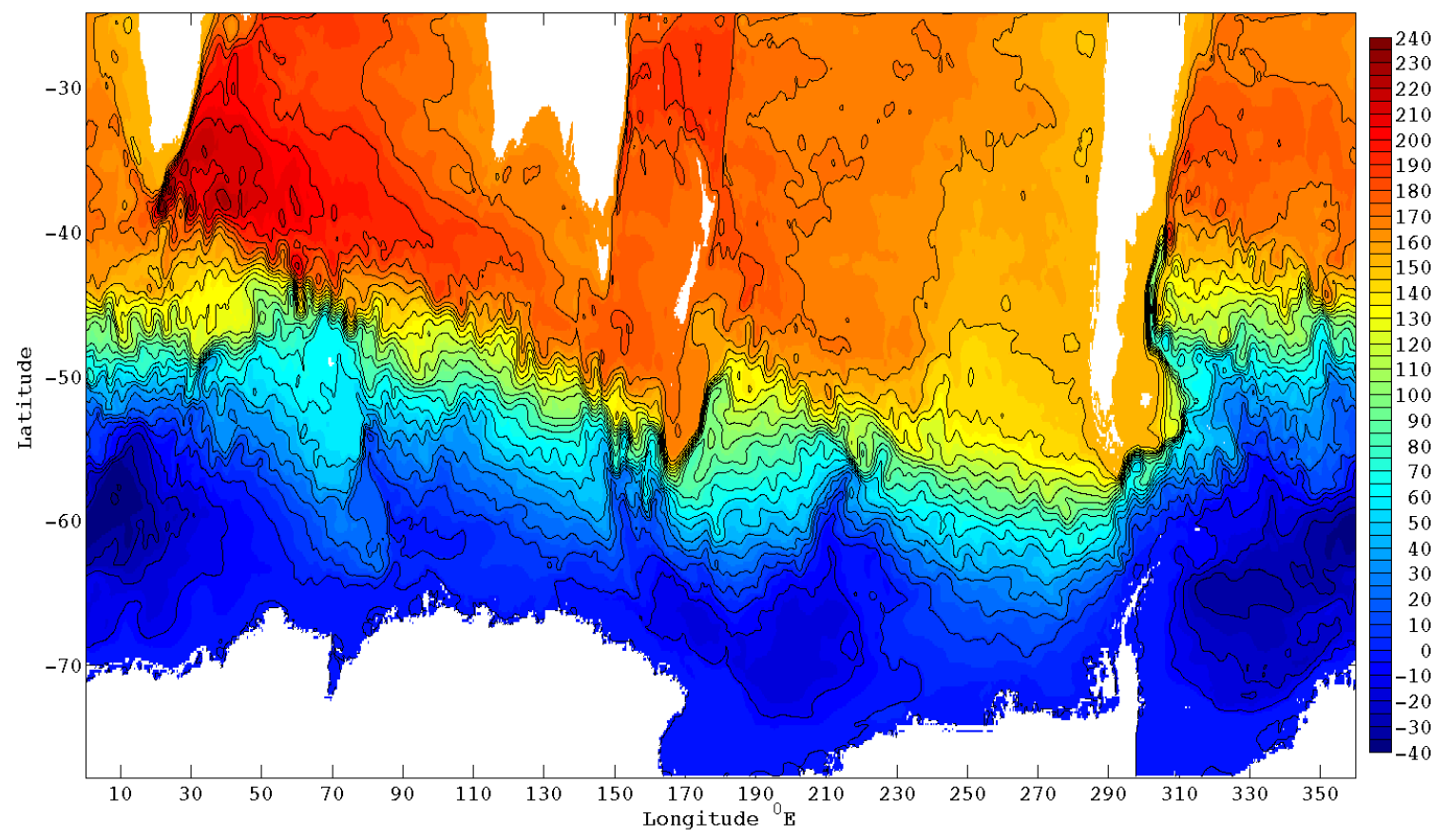

Figure 3-2: Mean vertically integrated transport streamfunction in Sverdrups from the Southern Ocean state estimate. The zero contour is the Antarctic coast, and the contour interval is $10 \mathrm{~Sv}$. At the top of the figure, in red-orange, are the anti-cyclonic subtropical gyres. The Indian Gyre's transport of $\sim 60 \mathrm{~Sv}$ is much stronger than the approximately $30 \mathrm{~Sv}$ transports of the Atlantic and Pacific Subtropical Gyres. At the bottom of the figure, in dark blue, are the cyclonic Polar Gyres. The Weddell Gyre $\left(295^{\circ} \mathrm{E}\right.$ to $\left.50^{\circ} \mathrm{E}\right)$ transport $(\sim 40 \mathrm{~Sv})$ is double the Ross Gyre $\left(150^{\circ} \mathrm{E}\right.$ to $\left.240^{\circ} \mathrm{E}\right)$ transport $(\sim 20 \mathrm{~Sv})$. Between the polar and subtropical gyres is the strong $(\sim 150 \mathrm{~Sv})$ eastward transport of the Antarctic Circumpolar Current System (in blue to orange). This current tracks poleward over the South Indian and South Pacific Oceans. Its equatorward excursions are abrupt, with the Falkland (or Malvinas) Current (off South America's eastern coast) being the most significant meridional shift.

\subsection{Transport}

\subsubsection{Vertically Integrated Transport Streamfunction}

The state estimate employs the Boussinesq approximation, and thus volume transport is the appropriate surrogate for mass transport. The mean Southern Ocean volume transport is shown (Figure 3-2) in terms of a vertically integrated streamfunction. This streamfunction, $\psi$, is calculated by taking the vertical integral of the mean zonal velocity, and then integrating meridionally from the pole to the latitude plotted. The 
continuity equation then shows that $U=-\psi_{y}$ and $V=\psi_{x}$, where $U$ and $V$ are the vertically integrated velocities. Thus the horizontal transport is found from the difference between two streamlines.

The cyclonic Southern Ocean polar gyres are found polewards of approximately $55^{0} \mathrm{~S}$. Their structure is shown (in blue) in Figure (3-2), and the maximum transport of these gyres is plotted in Figure (3-3). Early estimates of the Weddell Polar Gyre transport based on "absolute velocity measurements" suggested it was greater than 20 Sv (Reid, 1994), and likely somewhere between 30 to $50 \mathrm{~Sv}$ (Schroder and Fahrbach, 1999). A recent static inverse model finds a Weddell Gyre transport of $45 \mathrm{~Sv}$ (Sultan et al., 2007). The $40 \mathrm{~Sv}$ Weddell Gyre transport of the state estimate supports these results. Reid (1994) estimated the Ross gyre transport to be $20 \mathrm{~Sv}$ based on absolute geostrophic velocities, and Orsi et al. (1995) estimated a $10 \mathrm{~Sv}$ transport relative to $3000 \mathrm{~m}$. This is consistent with the $20 \mathrm{~Sv}$ transport found in the state estimate. Both polar gyres exhibit recirculations with slightly larger transports on their eastern flanks (see the double peak feature in their maximum mean transports: Figure 3-3). The similarity of this rather non-conventional recirculation in both polar gyres is worthy of investigation, especially since no reference regarding such a recirculation in the Ross Sea could be found. The westward boundary current that flanks much of the Antarctic continent is not apparent in Figure (3-2); the transport associated with this often swift current is rather small. The along-continent westward current is apparent, however, as part of the Ross and Weddell Polar Gyres.

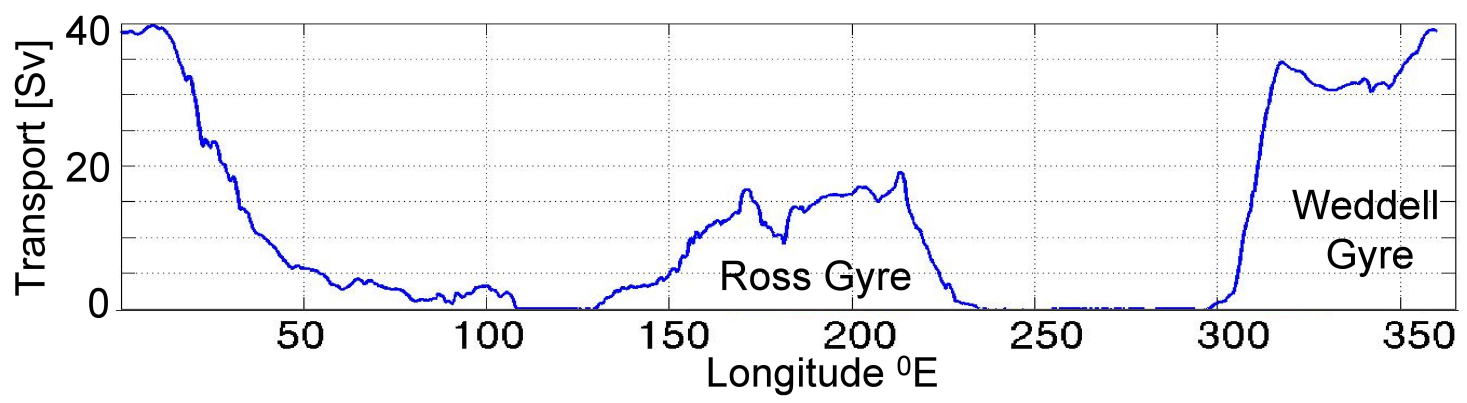

Figure 3-3: Maximum polar gyre transport in Sverdrups at each longitude in the state estimate. The Weddell Gyre transport is about $40 \mathrm{~Sv}$, while the Ross Gyre transport is about half that. The structure of both gyres can be seen in Figure (3-2). 


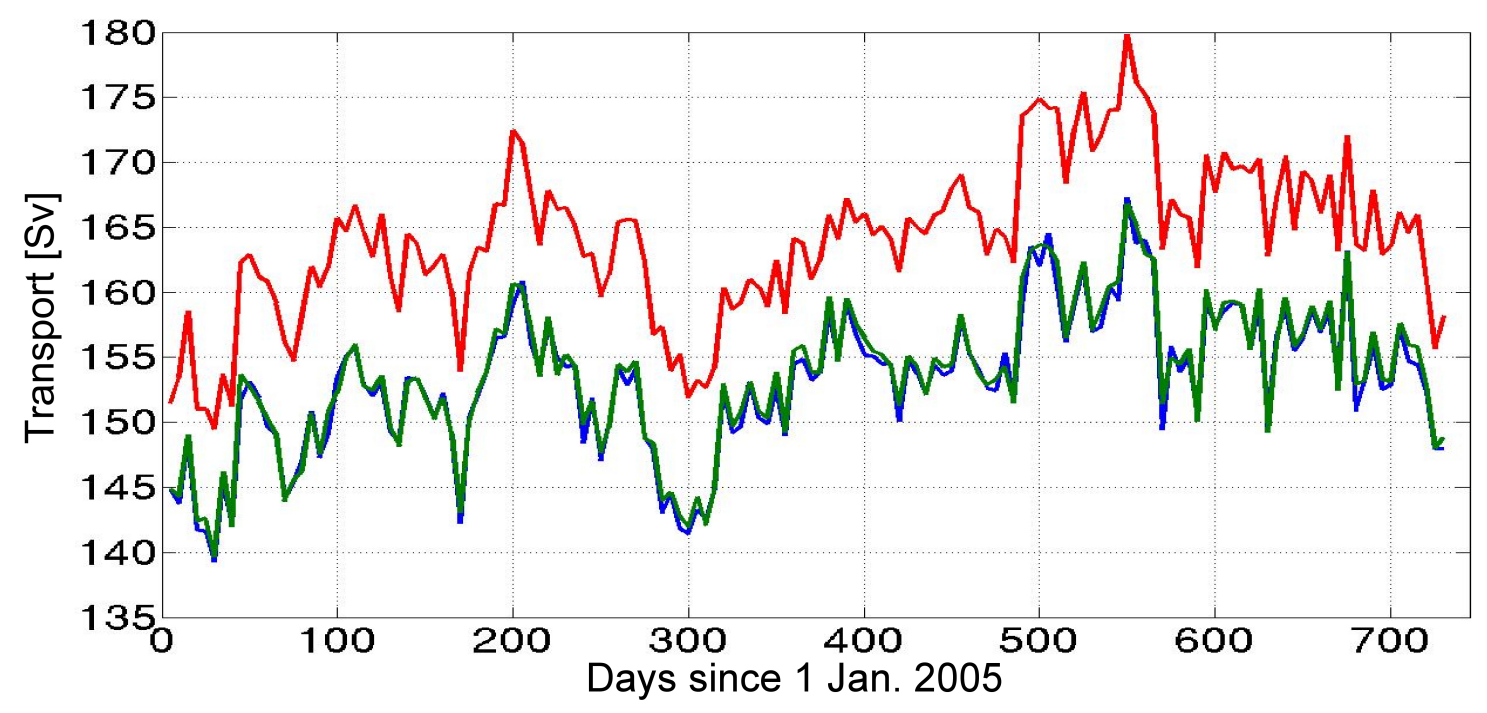

Figure 3-4: Time series of zonal transport through the WOCE Southern Ocean repeat sections, Drake Passage (SR1), Africa and Antarctica (SR2), and Australia to Antarctica (SR3), in Sv. The SR1 line has a mean transport of $153 \mathrm{~Sv}$ and a standard deviation of $5 \mathrm{~Sv}$ (blue). The SR2 line has a mean transport of $154 \mathrm{~Sv}$ and a standard deviation of $5 \mathrm{~Sv}$ (green). The SR3 line has a mean transport of $164 \mathrm{~Sv}$ and a standard deviation of $6 \mathrm{~Sv}$ (red).

The transports associated with the anti-cyclonic subtropical gyres can be seen (in red-orange) in the northern part of the domain of Figure (3-2). These gyres transport approximately $30 \mathrm{~Sv}$ in the Atlantic and Pacific Oceans. The Indian Ocean subtropical gyre is stronger, transporting about $60 \mathrm{~Sv}$. Between the anti-cyclonic sub-tropical gyres and the cyclonic polar gyres is the massive mean eastward transport of the Antarctic Circumpolar Current. The ACC transport is best observed at its three most constricted meridional sections: below Africa (the WOCE Southern Repeat section 2, SR2), below Tasmania (SR3), and below South America (SR1). The transport through these sections as a function of time (Figure 3-4) shows a significant seasonal cycle, and a slightly larger mean transport in 2006. This increase in transport is consistent with stronger mean winds and a larger Antarctic Oscillation index between $40^{\circ} \mathrm{S}$ and $70^{\circ} \mathrm{S}$ in 2006 (see the introduction to Chapter 2). The transport is larger through SR2 owing to the Indonesian Throughflow ( 11Sv). Besides this offset, however, the time-series track closely together suggesting minimal divergence and storage in each basin. 
The minimal variability in the ACC's divergence implies that while the ACC is not strictly a zonal flow, it is obviously circumpolar. Observation of its transport variability at any longitude gives one a good idea of its variability at all longitudes. The high-frequency transport magnitude signals travel much faster than the fluid moves itself. It is possible that the fastest fluid parcels moving on a direct path may travel from one section to the next in about a month. Figure (3-4) shows, however, that no significant time lag is present between sections. It is impossible that baroclinic Rossby waves could be transmitting this signal, since they propagate westward and are therefore slowed by advection from the eastward moving Antarctic Circumpolar Current. The high variability signal is due to barotropic ocean responses to atmospheric forcing. As these atmospheric signals move on order $10 \mathrm{~ms}^{-1}$, the circumpolar transport fluctuations have negligible lag on oceanic time-scales. This phenomenon has previously been reported to cause high variability in bottom pressure observations in the Drake Passage (Hughes et al., 2003).

The first modern estimate of the ACC transport came from the International Southern Ocean Study (ISOS). For this program, measurements from CTDs, current meters, and pressure gauges were collected in the Drake Passage over eight years (1974 to 1981). Studies analyzing these data have inferred large ranges of possible ACC transports (e.g. 28 to $290 \mathrm{~Sv}$ by Bryden and Pillsbury (1977) and 98 to $154 \mathrm{~Sv}$ by Whitworth, III and Peterson (1985)). The consensus that arose from this period, however, was a much more tightly constrained estimate that the annual mean ACC transport is approximately $134 \mathrm{~Sv}$ with a standard deviation of $11.2 \mathrm{~Sv}$ (Cunningham et al., 2003). A re-analysis on the ISOS data carried out by Cunningham et al. (2003) found that the canonical annual mean ACC transport value of $134 \mathrm{~Sv}$ was reasonable, but that error bounds for the estimate may be as large as $27 \mathrm{~Sv}$.

Static inverse models are consistent with the ISOS estimate, though they do tend to find slightly larger mean ACC transport values. For example, MacDonald and Wunsch (1996) obtained 142 Sv, Ganachaud and Wunsch (2000) obtained 140土6 Sv, and (Sloyan and Rintoul, 2001) find a transport of 135 Sv. The state estimate finds a two year mean ACC transport of $153 \mathrm{~Sv}$ (Figure 3-4), which is on the high side of 
the ISOS estimate, but within the error bounds prescribed by (Cunningham et al., 2003). Estimates South of Australia (SR3) have been found to be larger than SR1 by 10 to $20 \mathrm{~Sv}$ owing to the Indonesian Throughflow (e.g. Ganachaud and Wunsch (2000) find $157 \pm 10 \mathrm{~Sv}$ and Rintoul and Sokolov (2001) find approximately $147 \mathrm{~Sv}$ relative to the ocean bottom), which is consistent with the state estimate's value of $164 \pm 6$ Sv.

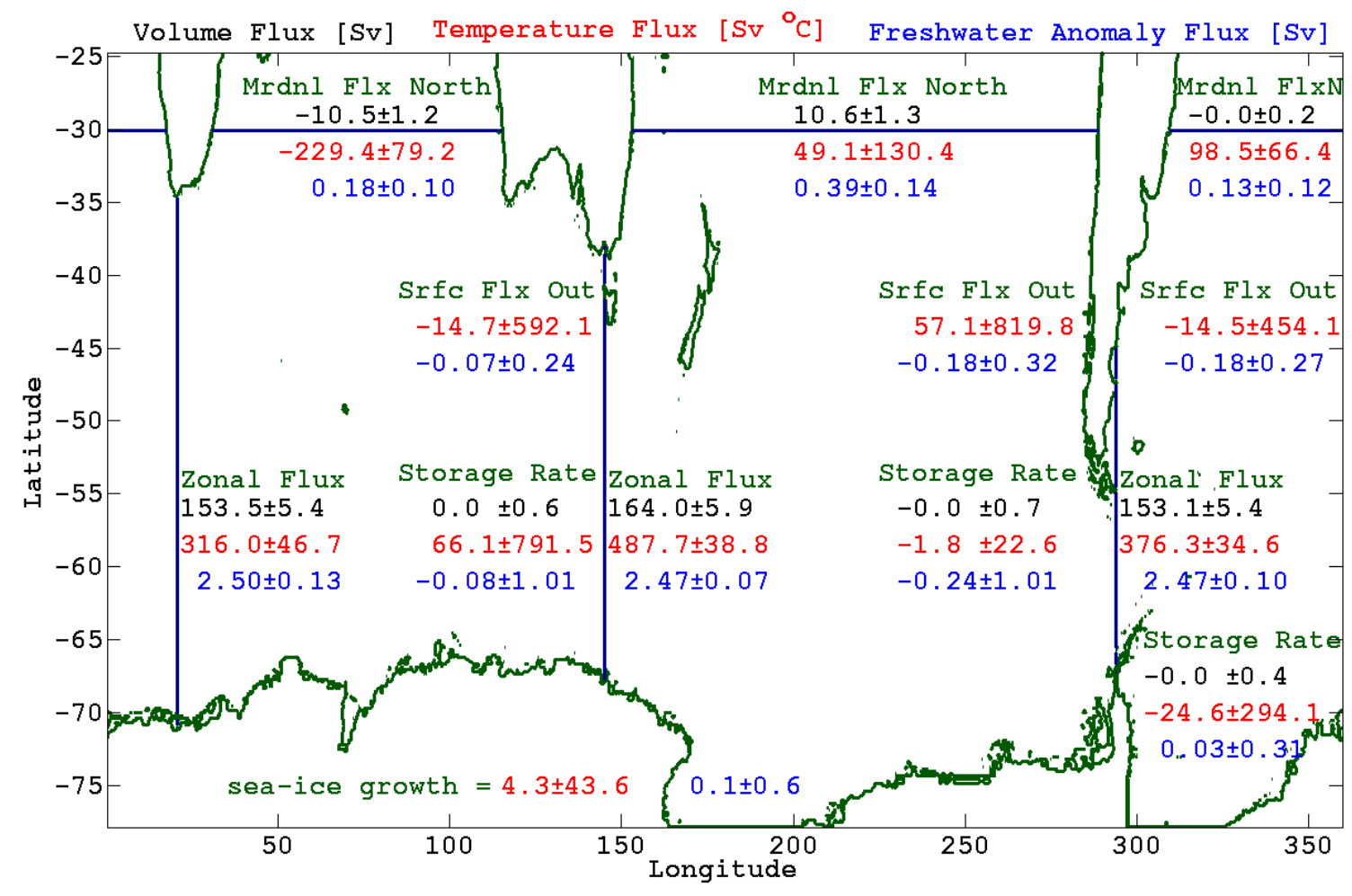

Figure 3-5: Volume flux $[\mathrm{Sv}]$ in black, temperature flux $\left[\mathrm{Sv}{ }^{0} \mathrm{C}\right]$ in red (to convert to approximate heat transports in petawatts multiply by $C_{p} \rho_{0} \times 10^{6} \approx 4.1 \times 10^{-3}$ $\left.\mathrm{PW}\left(\mathrm{Sv}^{0} \mathrm{C}\right)^{-1}\right)$, and freshwater anomaly flux $[\mathrm{Sv}]$ in blue (calculated with respect to 35 ) in the three Southern Ocean regions. The temporal standard deviations are given. Ocean waters are heated (cooled) and become more saline (fresh) when sea-ice is formed (melts), and this is accounted for by the noted sea-ice growth rate.

\subsubsection{Freshwater and Temperature Flux}

Using the WOCE Southern Repeat meridional sections, and the $30^{0} \mathrm{~S}$ zonal section, the Southern Ocean was separated into three regions. The time mean freshwater anomaly, temperature, and volume fluxes, and their standard deviations, in the South- 

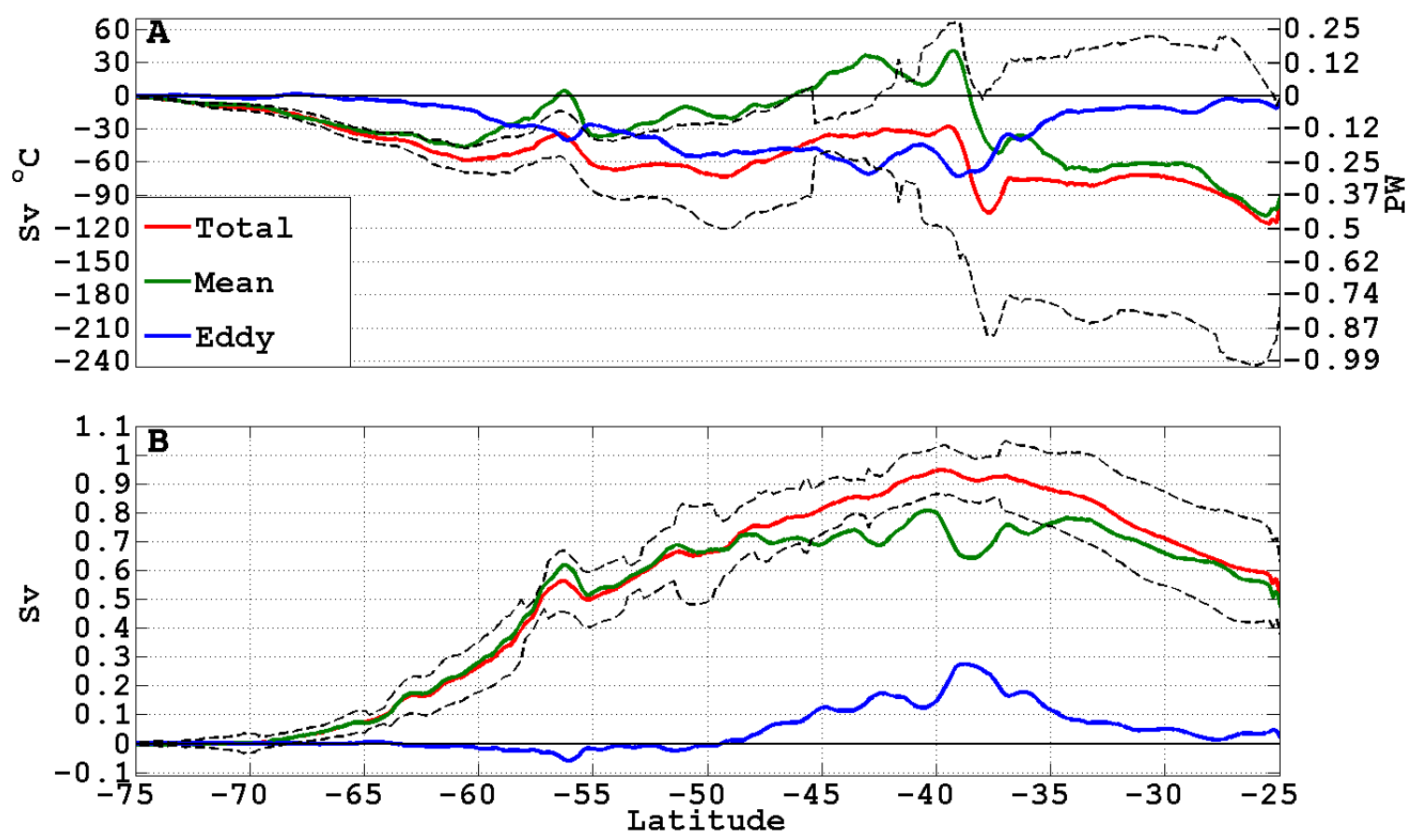

Figure 3-6: The northward (zonally integrated and time mean) meridional flux of heat in PW (Panel A) and freshwater anomaly (relative to 35) in Sv (Panel B) for the Southern Ocean state estimate. The time mean (green line), time variable (blue line), and total (red line) fluxes are given. Black dashed lines indicate the $95 \%$ confidence interval for the total flux. The number of degrees of freedom in the record was determined from evaluation of the decorrelation time scale at each latitude.

ern Ocean state estimate have been calculated for these regions (Figure 3-5). The net meridional volume flux from the Southern Ocean to the tropics is negligible, though about $11 \pm 1 \mathrm{~Sv}$ (note " \pm " values given in this section denotes temporal standard deviations) moves equatorward into the Pacific Ocean and returns from the Indian Ocean via the Indonesian Throughflow. This net poleward flow out of the Indian Ocean carries about $230 \pm 80 \mathrm{~Sv}{ }^{0} \mathrm{C}$, making it the primary source of temperature flux into the Southern Ocean. A mean northward flux of approximately $100 \pm 70 \mathrm{~Sv}{ }^{0} \mathrm{C}$ into the Atlantic Ocean and $50 \pm 130 \mathrm{~Sv}{ }^{0} \mathrm{C}$ into the Pacific Ocean and an approximately $40 \mathrm{~Sv}{ }^{0} \mathrm{C}$ net storage largely balances this input, leaving approximately $30 \mathrm{~Sv}{ }^{0} \mathrm{C}$ to be lost to surface cooling. The atmosphere temperature flux (surface cooling) in the Southern Ocean is, however, far from spatially uniform (Cerovecki et al., 2008).

The atmosphere warms the oceans in the South Atlantic and Indian Ocean regions (the combined temperature flux is $\sim-30 \mathrm{~Sv}{ }^{0} \mathrm{C}$ ). The net oceanic heat loss to the 

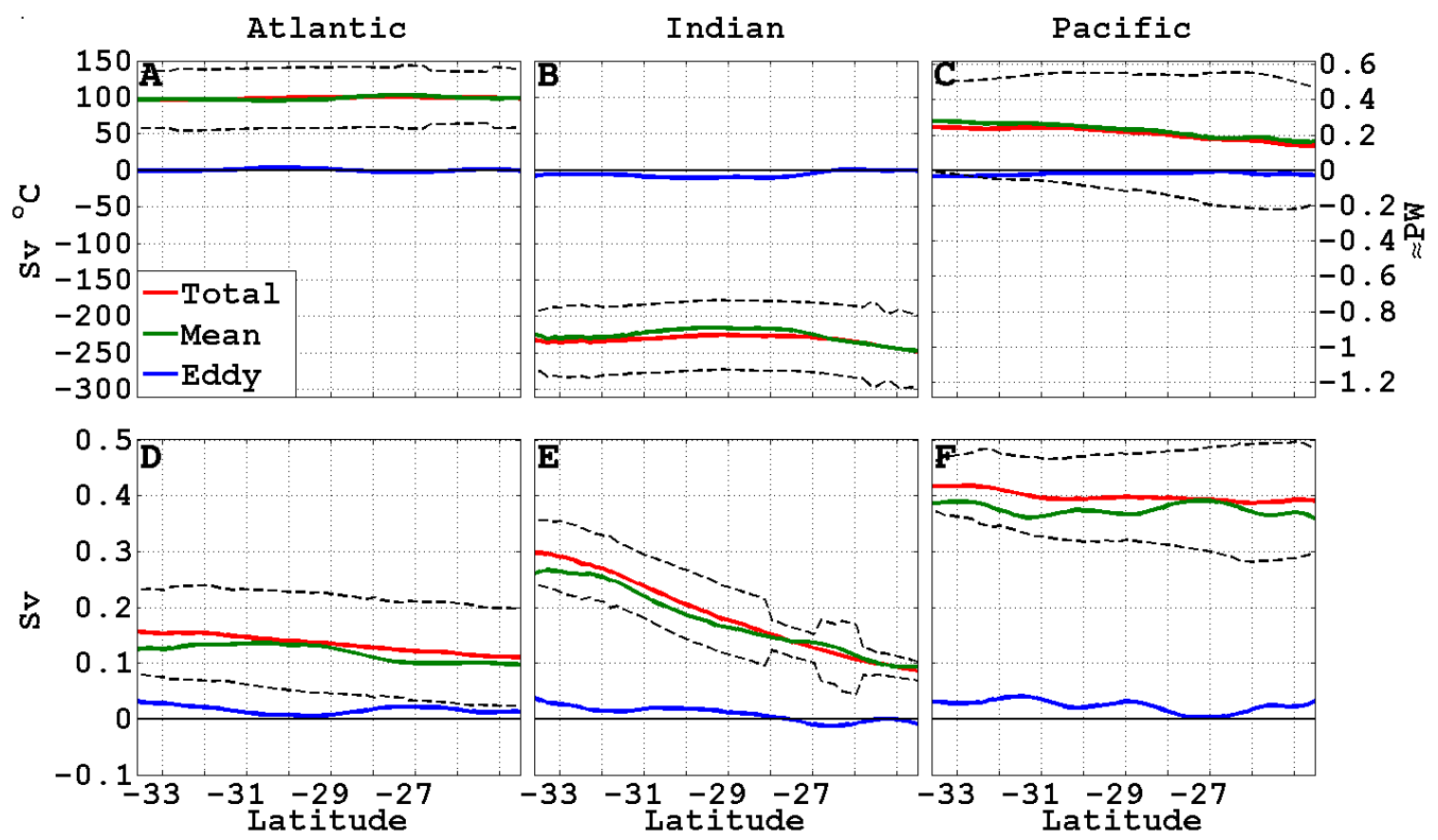

Figure 3-7: The northward (zonally integrated and time mean) meridional flux of temperature in $\mathrm{Sv}{ }^{0} \mathrm{C}$ (top panels) and freshwater anomaly (relative to 35 ) in $\mathrm{Sv}$ (bottom panels) for the Southern Atlantic, Indian, and Pacific Oceans. Except for the regional limits of the zonal integration, the figure is analogous to Figure (3-6). The volume flux across in these regional sections is non-zero, and thus the plots do not represent heat transports.

atmosphere is established in the South Pacific region, where the temperature flux of approximately $60 \mathrm{~Sv}{ }^{0} \mathrm{C}$ is larger than both the other regions combined. The ACC moves significantly southward in the Pacific region (Figure 3-2), suggesting a correlation between atmospheric heat fluxes and the ACC's meridional excursions. For the two year Southern Ocean state estimate, a temperature storage (warming) occurs in the South Indian Ocean region, while the South Atlantic region exhibits a cooling. The differences in the basin meridional temperature fluxes, storage rates, and surface fluxes result in large differences in the inter-basin zonal temperature fluxes. The temporal standard deviations of the temperature storage and atmospheric heat fluxes in the basins are an order of magnitude larger than the time mean.

In contrast to the temperature fluxes, the freshwater budget exhibits smaller differences between basins. The freshwater anomaly zonal fluxes through the sections are relatively steady at about $2.5 \pm 0.1 \mathrm{~Sv}$. Freshwater input from the atmosphere 
occurs in all regions, and this input is largely balanced by an equatorward export. The export to the tropics is most significant in the Pacific Ocean, likely due to the net volume flux out of this ocean along with its increased cross-sectional area. With a storage rate of $-0.24 \pm 1.01 \mathrm{~Sv}$, this South Pacific Ocean region loses the most freshwater during the two year estimate.

The northward (equatorward) meridional fluxes of freshwater anomaly and temperature are plotted as a function of latitude for global and regional zonal integrals (Figures 3-6 and 3-7). The fluxes have been decomposed into time mean and transient components: $\overline{v T}=\bar{v} \bar{T}+\overline{v^{\prime} T^{\prime}}$ and $\overline{v F}=\bar{v} \bar{F}+\overline{v^{\prime} F^{\prime}}$, where an overline represents a time mean and a prime represents the deviation from this mean. In the above equations $v$ is meridional velocity, $T$ is temperature, and $F \equiv(35-S) / 35$ is freshwater anomaly ( $\mathrm{S}$ is salinity). For the global integral, all latitudes have a poleward heat flux and an equatorward freshwater flux. The freshwater flux is carried primarily by the mean flow. The heat flux is carried by the time mean flow in the polar gyres (poleward of $\sim 60^{0} S$ ) and in the subtropical gyres (equatorward of $\sim 35^{0} S$ ). In the ACC latitudes, where the mean has a strong equatorward Ekman component, the transient dynamics are responsible for the poleward heat transport. The regional plots (Figure 3-7) are calculated in the subtropical gyres where the Ekman flow is poleward and the transient eddy component is rather insignificant. An interesting feature of these regional plots is the diminishing freshwater transport in the Indian region (Panel E). This pattern is indicative of significant evaporation in the region.

The Southern Ocean has a strong seasonal cycle, making it difficult to determine annual mean freshwater anomaly and temperature fluxes with much certainty. As noted above, the standard deviations for many of the budget terms (sea-ice growth, surface fluxes, storage rates) are larger than the time mean values, and thus the means are likely to represent variability in the seasonal cycle. To gain a better idea of the uncertainty in the meridional fluxes of Figures (3-6) and (3-7), the 95\% confidence interval was calculated. For each time series, the first zero crossing of the auto-correlation sequence is taken to be the decorrelation time-scale. The number of degrees of freedom in the records is determined by dividing the length of the record 
(two years) by the decorrelation time. The $95 \%$ confidence interval is then determined using the standard deviation of the record, the number of degrees of freedom, and by assuming the sampling is normally distributed about the mean.

The maximum number of degrees of freedom in the heat flux record occurs in the ACC latitudes between about $45^{0} S$ and $38^{0} S$. This is where the mean heat transport is equatorward, and the eddy heat flux is most significant. Decorrelation time for the heat flux drops to approximately 15 days in these latitudes, in contrast to an average decorrelation time of 92 days with a standard deviation of 40 days. The freshwater anomaly flux decorrelation time is also highly variable, with an average of 86 days and a standard deviation of 41 days. Minimas in the decorrelation times for the freshwater anomaly fluxes are, however, more evenly distributed spatially. One may deduce that high levels of eddy heat transport reduces the heat flux decorrelation time scale. It is not obvious what sets the decorrelation time scale for the freshwater anomaly flux.

Heat flux estimates at $\sim 30^{0} \mathrm{~S}$ are, for the most part, consistent with previous static inverse model estimates. The state estimate finds approximately $0.4 \mathrm{PW}$ fluxed into the Atlantic Ocean, Ganachaud and Wunsch (2000) find a flux of about 0.3 PW, Wijffels et al. (2001) find 0.4 PW, and Talley (2003) finds about 0.23 PW. The combined heat flux into the Indian and Pacific Oceans in the state estimate is about -0.8 PW. For this region Ganachaud and Wunsch (2000) and Wijffels et al. (2001) both find -0.9 PW, and Talley (2003) finds about -1.2 PW. For a total cooling over the Southern Ocean Ganachaud and Wunsch (2003) find -0.6 \pm 0.3 PW, Wijffels et al. (2001) find 0.46 PW, and Talley (2003) finds 0.8 PW. The heat loss in the state estimate is slightly under 0.5 PW.

The state estimate heat and freshwater transports are consistent with the $1 / 12^{0}$ global Ocean Circulation and Climate Advanced Model (OCCAM) in the ACC region where eddy transports are most important. The state estimate transports are, however, much larger in the subtropical and polar gyre regions (Lee et al., 2007). This is an interesting finding as it lends support to the ability of the state estimate to resolve eddies, while pointing to issues with the large scale forcing of the unconstrained 
OCCAM model.

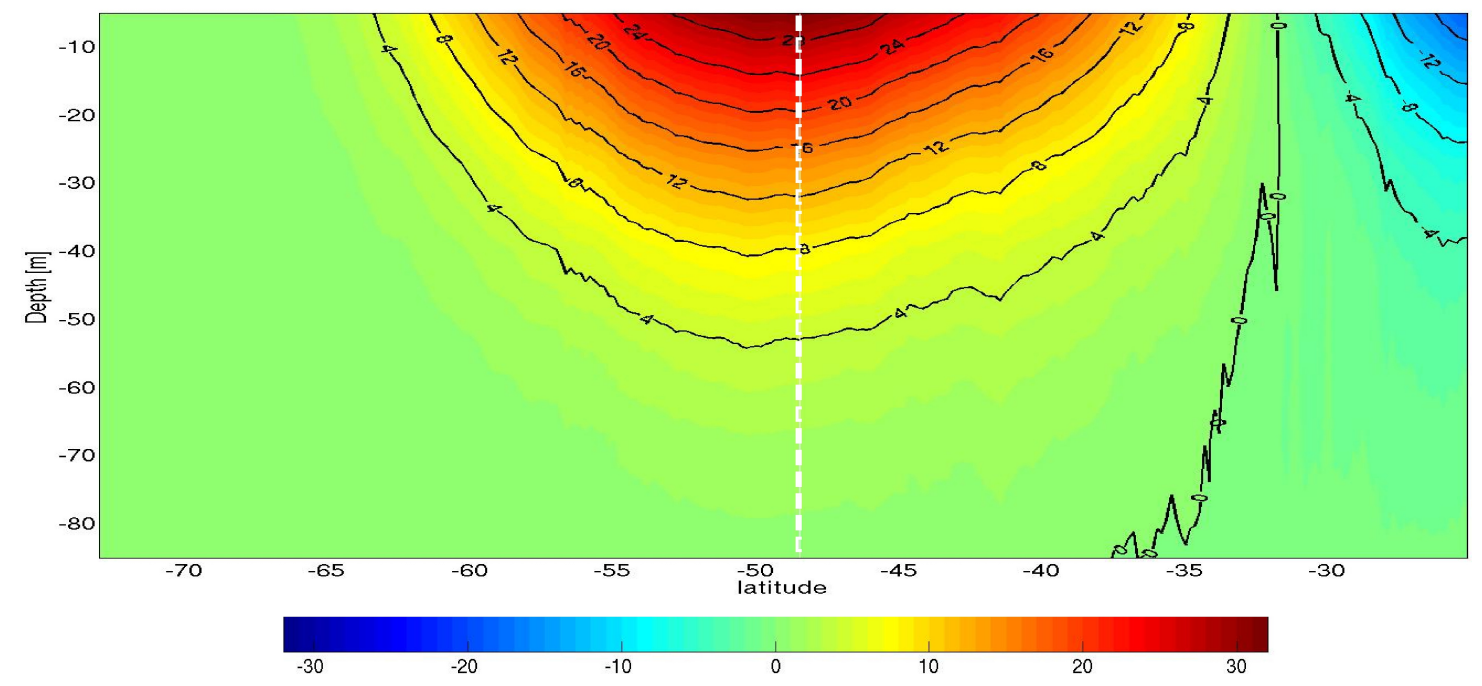

Figure 3-8: Ekman transport streamfunction in Sverdrups. The transport shown at each depth results from integrating the time averaged and zonally integrated ageostrophic velocity from 100 meters to the depth level shown. The white line denotes the latitude of maximum transport.

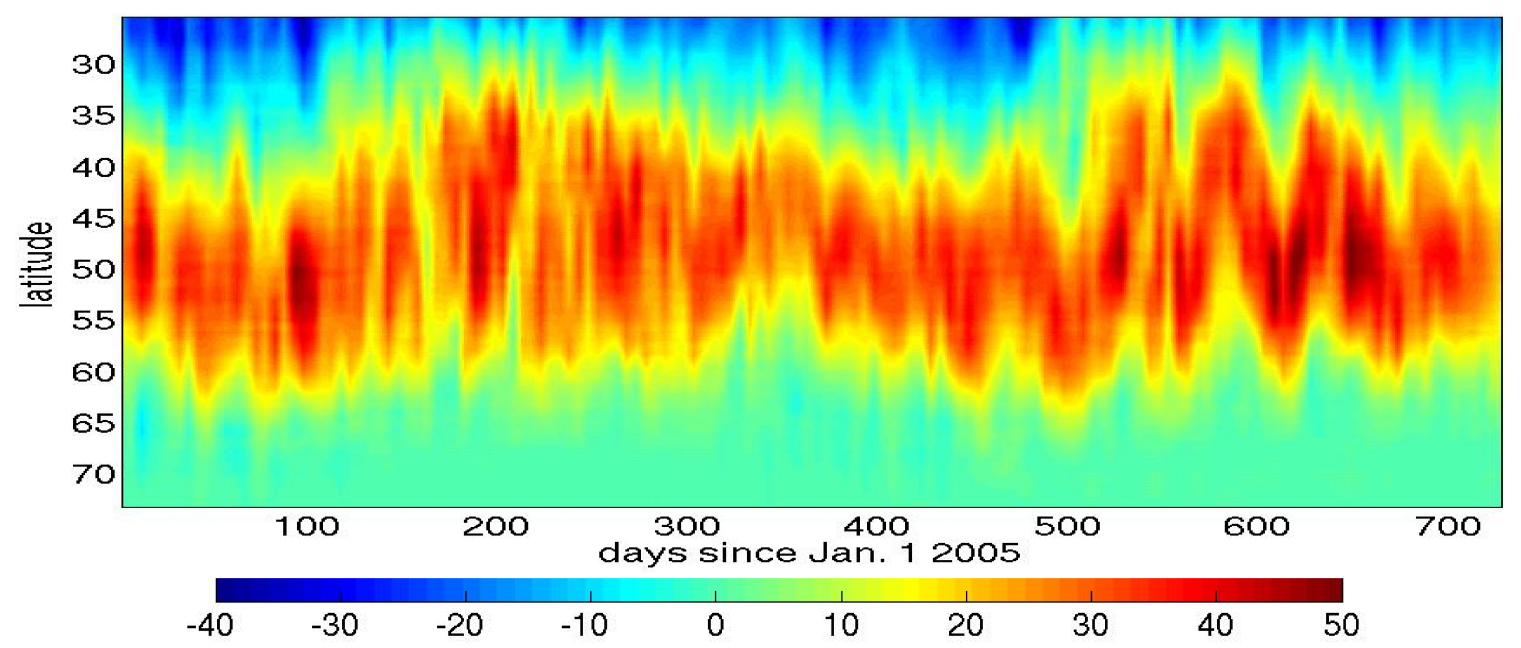

Figure 3-9: Zonally integrated Ekman transport in Sverdrups.

\subsubsection{Ekman Transport}

The winds drive a substantial meridional Ekman transport in the Southern Ocean. Analyses of various wind products estimate this transport to be between 20 and $30 \mathrm{~Sv}$ (Pickard et al., 2008). The maximum ageostrophic transport in the top 100 
meters of the state estimate is $32 \mathrm{~Sv}$, and it is assumed that this transport arises from wind driven Ekman layer dynamics. The ACC is primarily geostrophic. There are, however, a few regions of interaction between the ACC and topography where strong ageostrophic flows may arise from mechanisms besides wind stress. The most significant of these is the Falkland (or Malvinas) Current found to the east of South America. The net ageostrophic surface flow (top 100 meters) in this region (between $70^{\circ} \mathrm{W}$ and $43^{\circ} \mathrm{W}$ ) is order $1 \mathrm{~Sv}$. Though topographically complex regions in the Southern Ocean tend to have relatively small ageostrophic surface flows, these flows should not be ignored as they are primarily oriented in the northward direction (see the flow east of South America $\left(300^{\circ} \mathrm{E}\right)$ or the flow near the Campbell Plateau $\left(170^{\circ} \mathrm{E}\right)$ in Figure 3-2). Thus the magnitude of the northward transport shown in Figure (38 ) is likely an upper bound on the time-mean wind-driven Ekman transport. This transport is estimated to be between 30 and $32 \mathrm{~Sv}$.

A white line is drawn at the latitude of maximum transport, $\sim 48^{0} \mathrm{~S}$, which also happens to be the approximate latitude of the Subantarctic Front. South of this latitude is a region of Ekman divergence, and it is here that UCDW is upwells. North of this latitude is a region of convergence, and here AAIW is subducted. A significant time-mean poleward Ekman transport is absent close to the Antarctic continent where easterly winds preside.

The strength and location of the core of the westerly winds has a large seasonal fluctuation, and this is apparent in the Ekman transport (Figure 3-9). In the winter this transport reaches up to $50 \mathrm{~Sv}$, much larger than the seasonal average of approximately 32 Sv. Figure (3-9) also shows that there is Ekman transport variability at frequencies higher than the seasonal cycle. The power density spectrum of this transport (not shown) exhibits a strong peak at the seasonal cycle, $\sim 90$ days, and even stronger peaks between 55 and 65 days. These peaks, and several smaller ones at even higher frequency, are essentially identical to energy peaks in the wind stress. The high frequency variability in the ageostrophic transport is wind driven. 


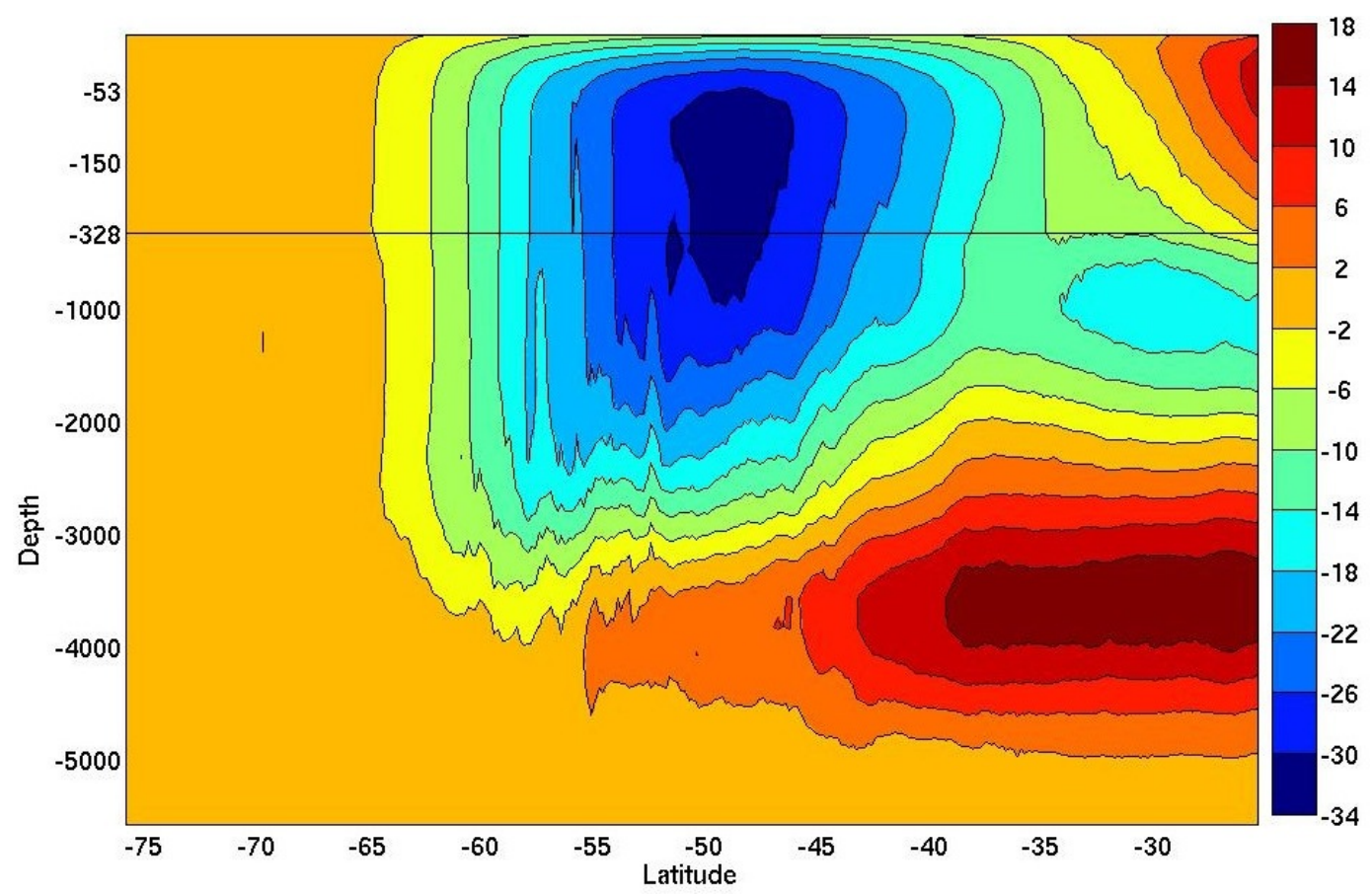

Figure 3-10: Southern Ocean overturning streamfunction in Sverdrups. The contour interval is $4 \mathrm{~Sv}$ starting at \pm 2 . Note the vertical scale is different for depths above 328 meters. The negative values denote clockwise flow, while the positive values denote counter-clockwise flows.

\subsubsection{Meridional Overturning Streamfunction}

To quantify the transport of water masses it is useful to calculate the meridional overturning streamfunction, $\Psi$, by integrating the meridional velocity zonally, and then vertically from the surface to a depth, $z$. The continuity equation shows $v=\Psi_{z}$, and $w=-\Psi_{y}$. The streamfunction, plotted in Figure (3-10), is determined to within a constant, and it is important to remember that actual transports are given by the difference between streamline values.

The strong Ekman transport is apparent in Figure (3-10), and it is supplied from depths reaching greater than $3000 \mathrm{~m}$. This strong overturning (the so-called Deacon Cell), however, flows mostly along density surfaces; it is primarily an adiabatic circulation (Döös and Webb, 1994). Taking the path of zonal integration along isopycnals helps to demonstrate this. The mean volume weighted overturning circulation is plotted in isopycnal space in Figure (3-11). In this work, neutral density, $\gamma$, has been used in an attempt to limit compressibility effects in the isopycnal coordinate. 


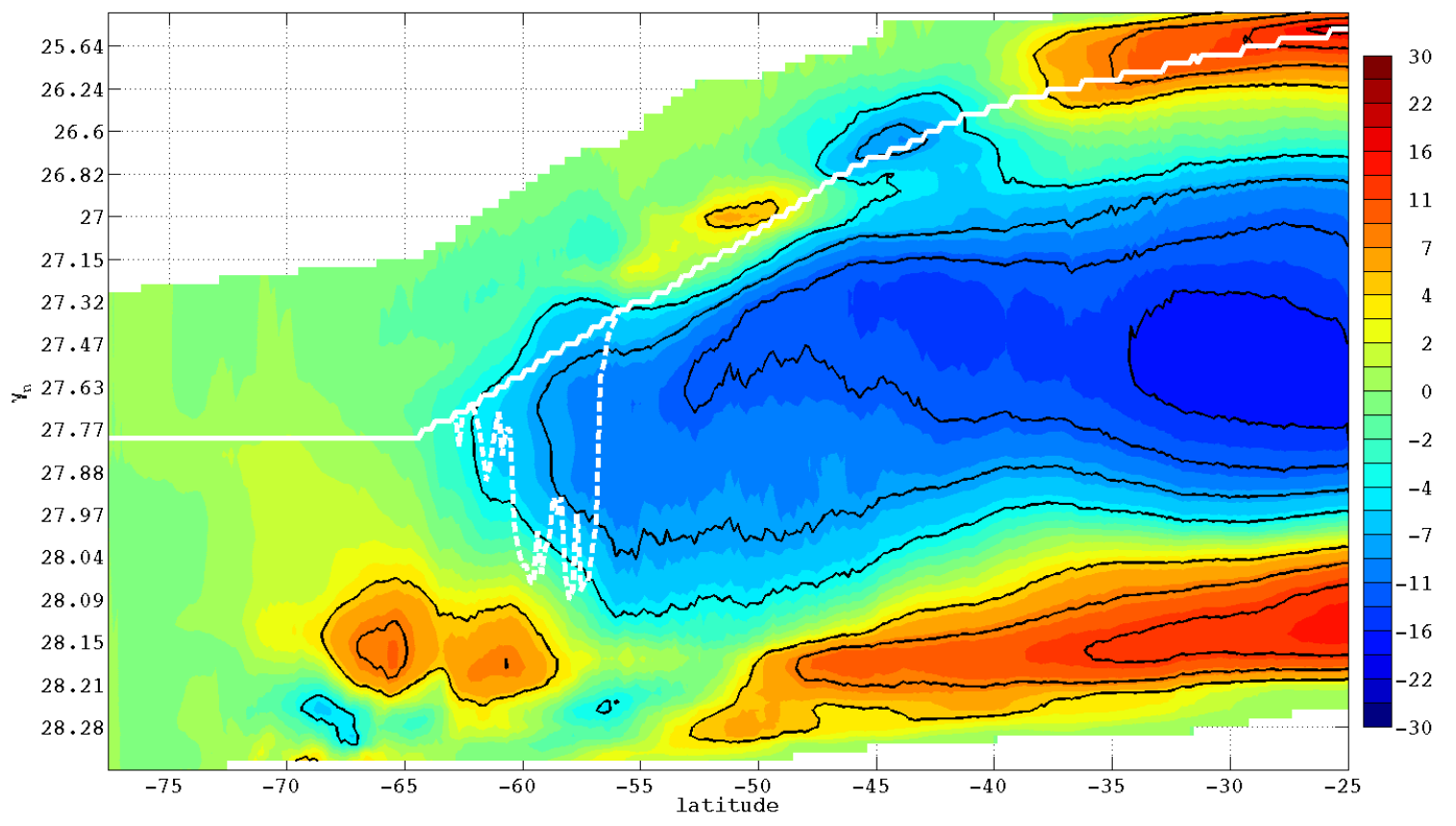

Figure 3-11: Southern Ocean overturning streamfunction [Sv] plotted in neutral density, $\gamma$, space. The contour interval is $4 \mathrm{~Sv}$ (starting at \pm 4 , the zero contour has been omitted). Positive (negative) values denote counter-clockwise (clockwise) circulations. Vertical deviations of streamlines denote movement across isopycnals, and thus that diabatic processes are occurring. The solid white line shows the approximate mean location of the surface. The dashed white line shows the approximate bounds of latitude circles that do not intersect bottom topography. Note the ordinate is stretched in $\gamma$ space by a factor that reflects the volume of water at each density class (i.e. approximately the same value of water is found between each $\gamma$-tick mark). The color axis is also stretched.

Neutral density is a locally referenced potential density, defined such that changes in $\gamma$ are only due to changes in temperature and salinity; compressibility effects have been removed: $\nabla \rho=\nabla \gamma-\Gamma \nabla p$ (see McIntosh and McDougall (1996); Jackett and McDougall (1997) or Section 6.5 of Stewart (2005)). The northward transport of the buoyant surface waters is order $10 \mathrm{~Sv}$ between $40^{\circ} \mathrm{S}$ and $45^{\circ} \mathrm{S}$, and between $55^{\circ} \mathrm{S}$ and $60^{0} \mathrm{~S}$. Perhaps more surprising is that the net transport of these waters is approximately $5 \mathrm{~Sv}$ poleward between $45^{0} \mathrm{~S}$ and $55^{0} \mathrm{~S}$. The Deacon Cell has been reduced or eliminated, showing that most of the strong Ekman transport returns along the same isopycnals (this flow is discussed further in Section 4.3). In the isopycnal average the lower cell of the streamfunction is interrupted in the Drake Passage latitude band, 


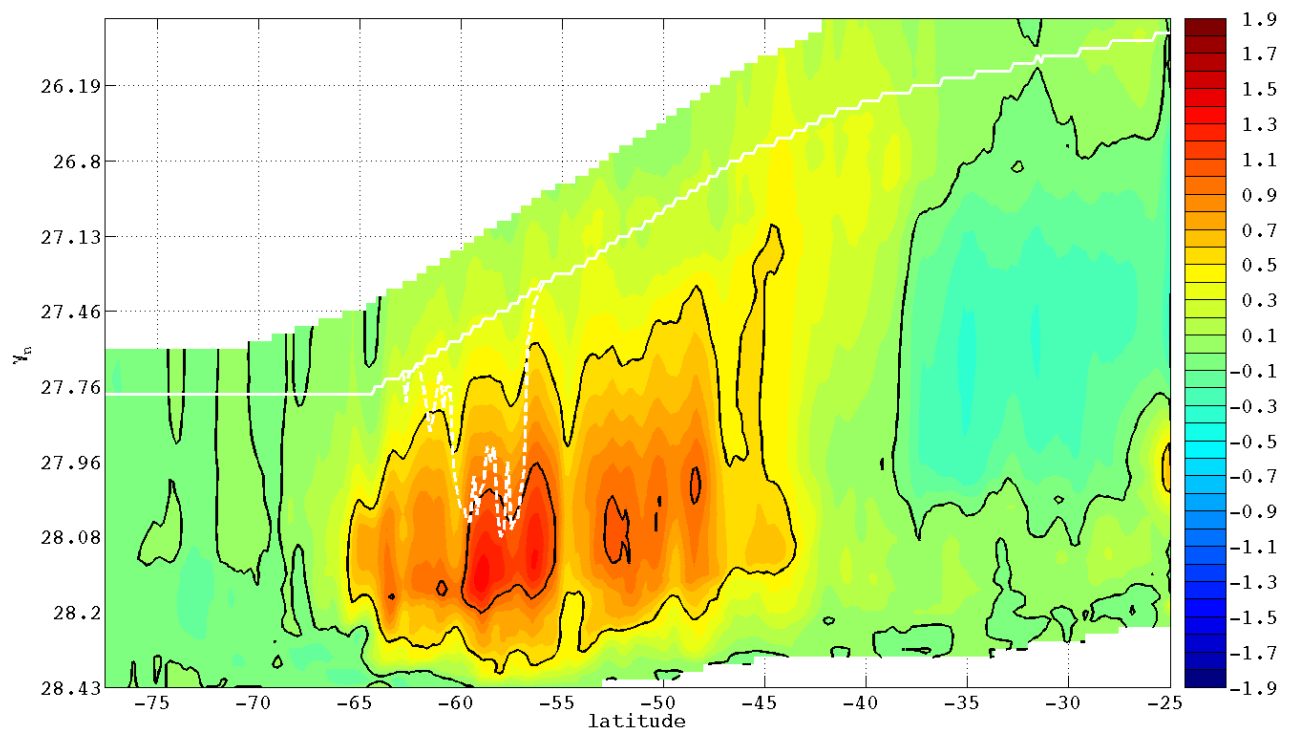

Figure 3-12: The time mean overturning streamfunction [Sv] parameterized by the Gent-McWilliams package. The plot is analogous to Figure (3-11), exceptions being that in this plot the contour interval is $0.5 \mathrm{~Sv}$ and the color-axis range is smaller.

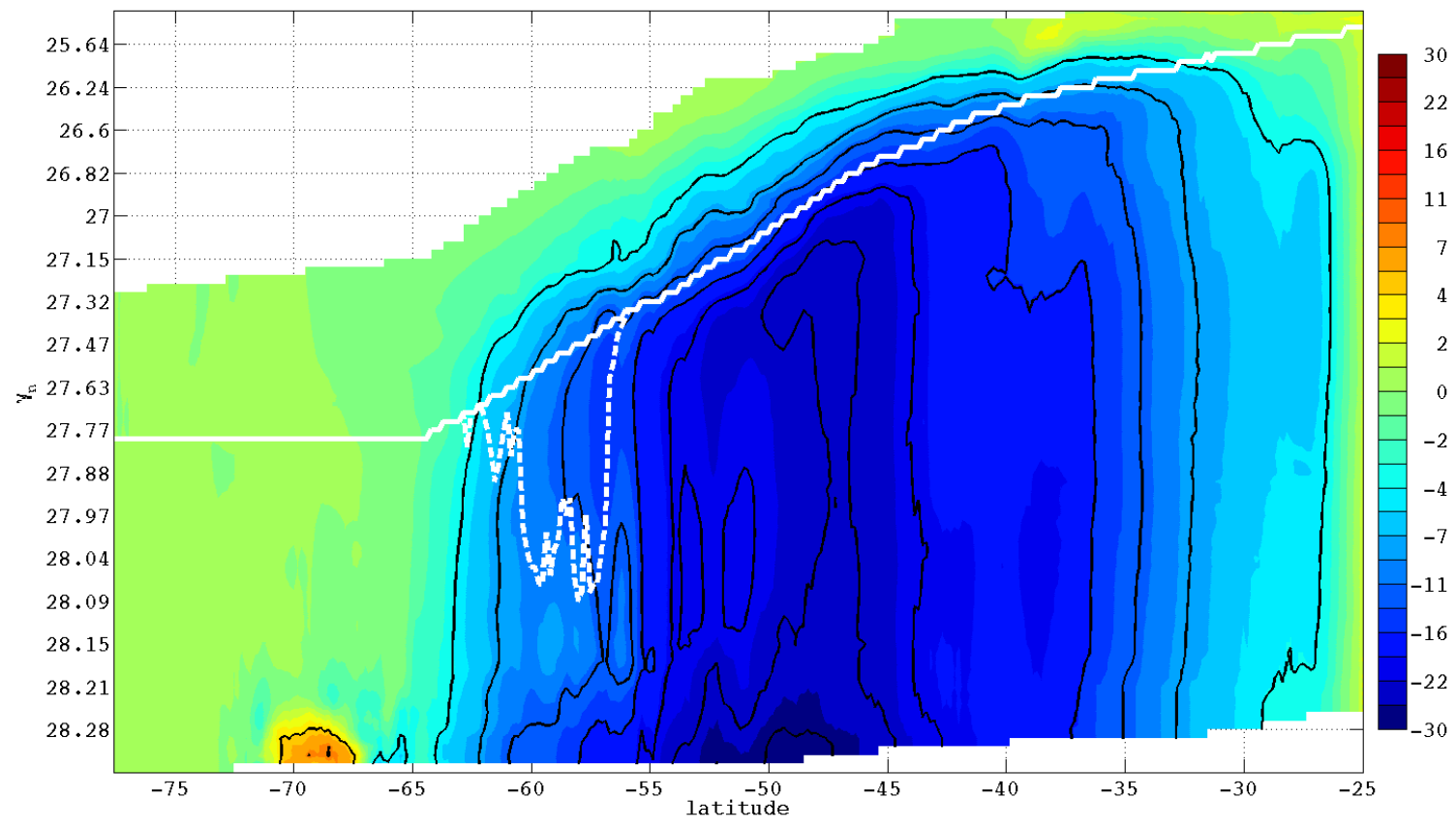

Figure 3-13: Eddy component of Southern Ocean overturning streamfunction [Sv] plotted in $\gamma$ space. The plot is comparable to Figure (3-11). 


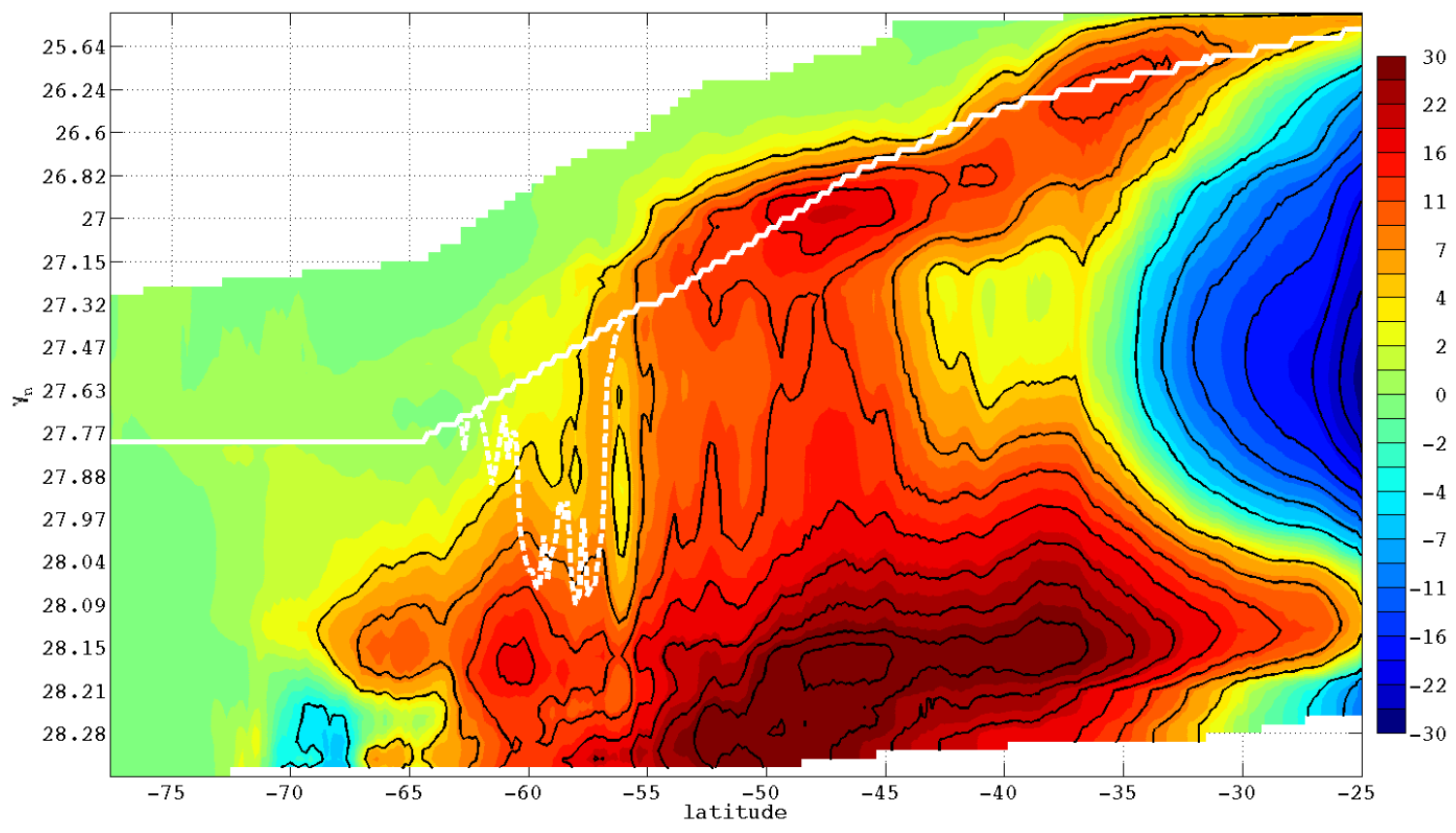

Figure 3-14: Mean component of Southern Ocean overturning streamfunction [Sv] plotted in $\gamma$ space. The plot is comparable to Figure (3-11).

and this is due to a transient adjustment of the model (a drift). Some water density classes are thickening, and this thickening in the presence of the meridional velocities significant vertical shear affects the temporally averaged streamfunction. This is discussed in detail in Section (4.3.6).

The streamfunction shown in Figure (3-11) is the advective volume transport at each density class. The Southern Ocean state estimate uses the Gent-McWilliams (GM) parameterization to represent the effect of unresolved eddy advective fluxes of temperature and salinity. If the meridional overturning streamfunction given in Figure (3-11) is to be interpreted as the residual overturning circulation (i.e. the overturning circulation that advects ocean properties, see Section 4.1.3), then the GM parameterized component of advection must be included. Comparison of the overturning circulation imparted by this parameterization (Figure 3-12) to the resolved overturning circulation (Figure 3-11) shows, however, that this parameterization drives a transport of less than $1 \mathrm{~Sv}$, and is therefore negligible. ${ }^{1}$ Though Figure

\footnotetext{
${ }^{1}$ Because the Southern Ocean state estimate is eddy permitting, the GM mixing coefficient was chosen to be small, and this is primarily why the GM parameterized overturning streamfunction is
} 
(3-11) omits the GM parameterization, to lowest order it can be interpreted as the residual overturning circulation.

Transient dynamics play a large role in the meridional circulation, as is apparent from the eddy component of the overturning streamfunction (Figure 3-13). Here the eddy component is defined as the temporal correlation contribution between meridional velocity and layer thickness, $\overline{v^{\prime} h^{\prime}}=\overline{v h}-\bar{v} \bar{h}$. To calculate this eddy overturning streamfunction, the model state was sampled by taking five day means. This sampling interval is sufficient to capture eddy dynamics, because altimeter data indicates an e-folding scale of 34 days for temporal variability in the Southern Ocean (Gille, 1995). As the eddy overturning streamfunction reflects correlated densities and velocities that deviate from the estimate's two year mean, it includes the effects of both atmospheric forcing variations and turbulent dynamics. Zonal integration is carried out after temporal averaging and differencing of the terms.

The transient dynamics are responsible for an approximately 12 to $24 \mathrm{~Sv}$ equatorward transport of surface density class waters between $35^{\circ} \mathrm{S}$ and $60^{\circ} \mathrm{S}$ (Figure 3-13). This flow works in concert with the equatorward Ekman flow, a result that is explained in Section (4.3.8) by consideration of the mean PV gradient. There is some structure to the transient dynamics away from the surface: the eddy streamfunction diminishes in the intermediate to deep density classes, but then increases again to transport a significant amount of deep water equatorward across $48^{0} \mathrm{~S}$. The mean meridional overturning streamfunction is in opposition to the eddy streamfunction (Figure 3-14). There is strong poleward transport in the near surface and circumpolar deep water density classes. The transport drops off noticeably in the Drake Passage latitudes. It is obvious that this mean streamfunction is not due simply to Ekman layer dynamics. This structure is explained in Chapter 4 (see the discussion of $\Psi_{g}$ in Section 4.4).

The meridional overturning streamfunction plotted in isopycnal and vertical coordinates (Figures 3-10 and 3-11) enables one to draw a qualitative picture of the meridional circulation. Upper Circumpolar Deep Water (UCDW) enters the Southan order of magnitude less than the resolved one. 
ern Ocean ${ }^{2}$ between approximately 1,000 and 2,500 meters. This water upwells between $30^{\circ} \mathrm{S}$ and the Drake Passage, mixing to help form Antarctic Intermediate Water (AAIW). AAIW flows equatorward near the surface until convergence at the Subtropical front, at approximately $37^{0} \mathrm{~S}$. Poleward flowing surface waters from the subtropical gyres are also subducted at the Subtropical Front. These waters join the AAIW in a strong equatorward flow. A significant portion of the upwelled UCDW never crosses $40^{\circ} \mathrm{S}$ to enter the Antarctic Circumpolar Current. At depths below 2,500m flow does cross the ACC fronts, and one can see Lower Circumpolar Deep Water (LCDW) extending poleward into the polar gyres. LCDW mixes with the cold, dense waters of these gyres to form AABW and contribute to an approximately $20 \mathrm{~Sv}$ deep overturning circulation. Approximately $13 \mathrm{~Sv}$ of AABW flows equatorward out of the Southern Ocean.

The deep overturning across $32^{0}$ in the state estimate of approximately $13 \mathrm{~Sv}$ is slightly lower than previous estimates from static inverse models (about $22 \pm 5 \mathrm{~Sv}$ from Talley et al. (2003) and about 14 Sv from Lumpkin and Speer (2007)). The intermediate overturning is approximately $17 \mathrm{~Sv}$, and this value is slightly larger than previous estimates (e.g. Talley (2003) finds an overturning of about $12 \mathrm{~Sv}$ and Lumpkin and Speer (2007) find about $13 \mathrm{~Sv}$ ). The upper overturning cell is approximately $12 \mathrm{~Sv}$ across $32^{0}$, also slightly larger than the previous static inverse model estimates (e.g. Lumpkin and Speer (2007) finds an overturning of about $7 \mathrm{~Sv}$ ). These inconsistencies, however, are not alarming. The state estimate is within about $5 \mathrm{~Sv}$ of the overturning inferred from the static inverse estimate of Lumpkin and Speer (2007). The differences are larger with respect to the static inverse estimate of Talley (2003), but still reasonable as the magnitude of the inferred overturning from a static inverse model varies significantly depending on the density bins (resolution) chosen.

The overturning streamfunction found in the state estimate is in reasonable agreement with those found in FRAM (Döös and Webb, 1994) and OCCAM (Lee and Cow-

\footnotetext{
${ }^{2}$ For brevity the term "enters (leaves) the southern ocean" will be used synonymously with flows poleward (equatorward) across $30^{\circ} \mathrm{S}$.
} 
ard, 2003). One difference is the state estimate finds a stronger overturning at depth than that of FRAM. Also FRAM and OCCAM both find a negligible overturning poleward of $65^{0} \mathrm{~S}$, whereas the state estimate finds an overturning of approximately $20 \mathrm{~Sv}$. This value is largely consistent with the magnitude of deep water formation inferred from static inverse models (about $15 \mathrm{~Sv}$ in Lumpkin and Speer (2007) and 21 \pm 6 Sv in Ganachaud and Wunsch (2000)), suggesting inaccuracies in the unconstrained models.
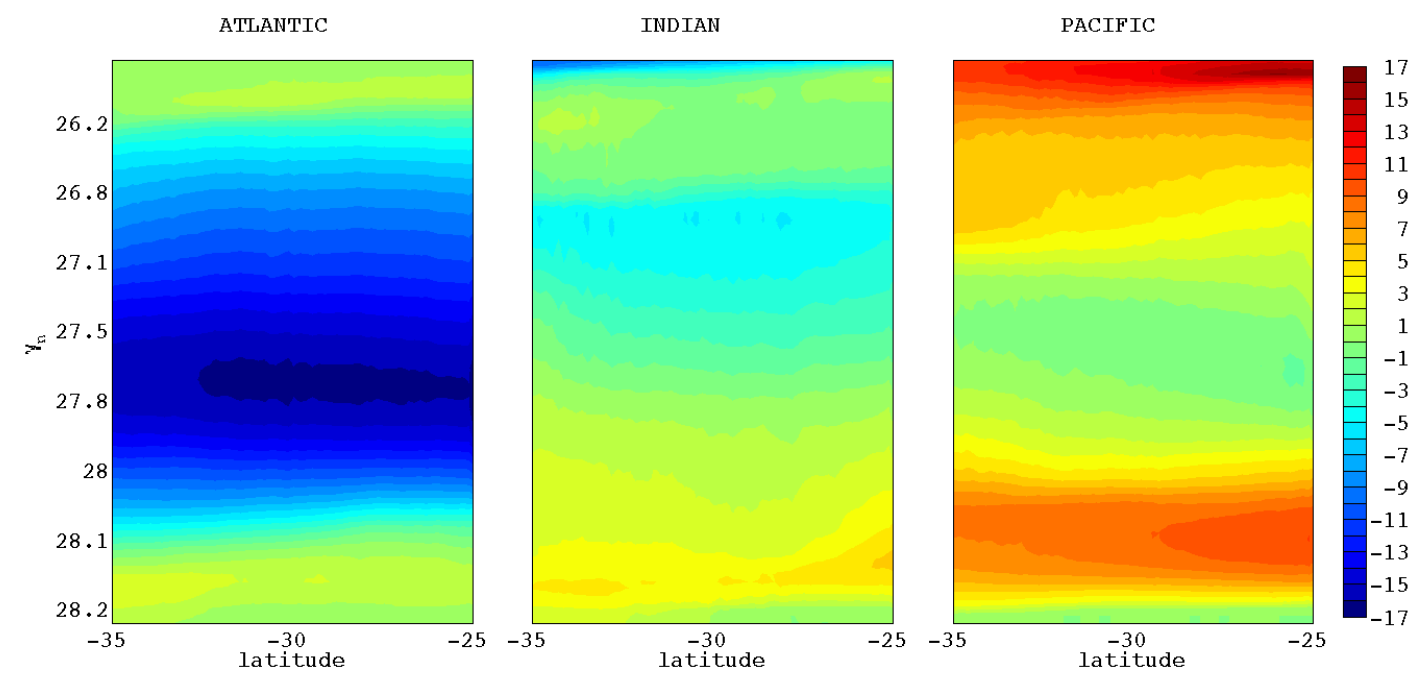

Figure 3-15: Overturning streamfunction [Sv] for the South Atlantic, South Indian, and South Pacific Oceans plotted in neutral density, $\gamma$, space. Positive (negative) values denote counter-clockwise (clockwise) circulations. Note the ordinate is stretched in $\gamma$ space. The color axis is also stretched.

The meridional overturning streamfunction in the southern regions of the Atlantic, Indian, and Pacific Oceans is considered to approximate the transport of water masses flowing into and out of the Southern Ocean (Figure 3-15). In the South Atlantic there is an abyssal outflow of AABW of $\sim 5 \mathrm{~Sv}$. This is consistent with the static inverse estimates of Ganachaud and Wunsch (2000) and Lumpkin and Speer (2007), which find $6 \pm 1.3 \mathrm{~Sv}$ and 5.6 $\pm 3.0 \mathrm{~Sv}$ of AABW entering the Atlantic. These studies also find $23 \pm 3 \mathrm{~Sv}$ and $17.9 \pm 3.1 \mathrm{~Sv}$ of NADW entering the basin. The state estimate's $20 \mathrm{~Sv}$ transport is consistent with these inferences. The upper ocean estimated transport in the Atlantic is also consistent with these previous studies. 
The abyssal flow in the South Indian Ocean region is consistent with previous inferences, as the state estimate has about $6 \mathrm{~Sv}$ of AABW export, and Ganachaud and Wunsch (2000) found $8 \pm 4 \mathrm{~Sv}$. The other flow components are, however, rather different. Ganachaud and Wunsch (2000) infer that $3 \pm 5 \mathrm{~Sv}$ of deep water is flowing out of the Southern Ocean, while the state estimate finds that about $5 \mathrm{~Sv}$ of deep water is flowing into the Southern Ocean. The intermediate and surface flow is also quite different, partly because the Indonesian Throughflow estimate of Ganachaud and Wunsch (2000) is $15 \pm 5 \mathrm{~Sv}$, which is larger than the $11 \mathrm{~Sv}$ prescribed in the state estimate.

The abyssal transport in the South Pacific region of the state estimate is consistent with that inferred by Ganachaud and Wunsch (2000), but again, largely because of an Indonesian Throughflow discrepancy, the estimate differs in the more buoyant density classes. In Ganachaud and Wunsch (2000), Antarctic Bottom Water export to the Pacific Ocean is $7 \pm 2 \mathrm{~Sv}$, the deep water import is $9 \pm 3 \mathrm{~Sv}$, and the intermediate and surface water transport is $19 \pm 5 \mathrm{~Sv}$. In the state estimate the bottom water export and the deep water import are both about $10 \mathrm{~Sv}$, and the intermediate and surface water export is about $11 \mathrm{~Sv}$.

\subsection{Rate of Wind Work}

Most strong ocean flows are associated with western boundary currents, and thus have a predominantly north-south orientation. The ACC, however, is oriented roughly east-west and parallel to the strong zonal winds. For this reason, it is in the ACC latitudes where the majority of wind energy is transferred into the world's oceans (Wunsch, 1998). A recent calculation using the ECCO-GODAE state estimate found that the wind power input to the global ocean is approximately $1.9 \mathrm{TW}$. Using the Southern Ocean state estimate, it is found that the wind power input over the domain is about $1.2 \mathrm{TW}$; about $60 \%$ of the total estimated input (Figure 3-16). Of the 1.9 TW of power diagnosed from the global ECCO-GODAE state estimate, 0.9 TW is transferred to the ageostrophic flow, and the other 1.0 TW to the geostrophic flow. 


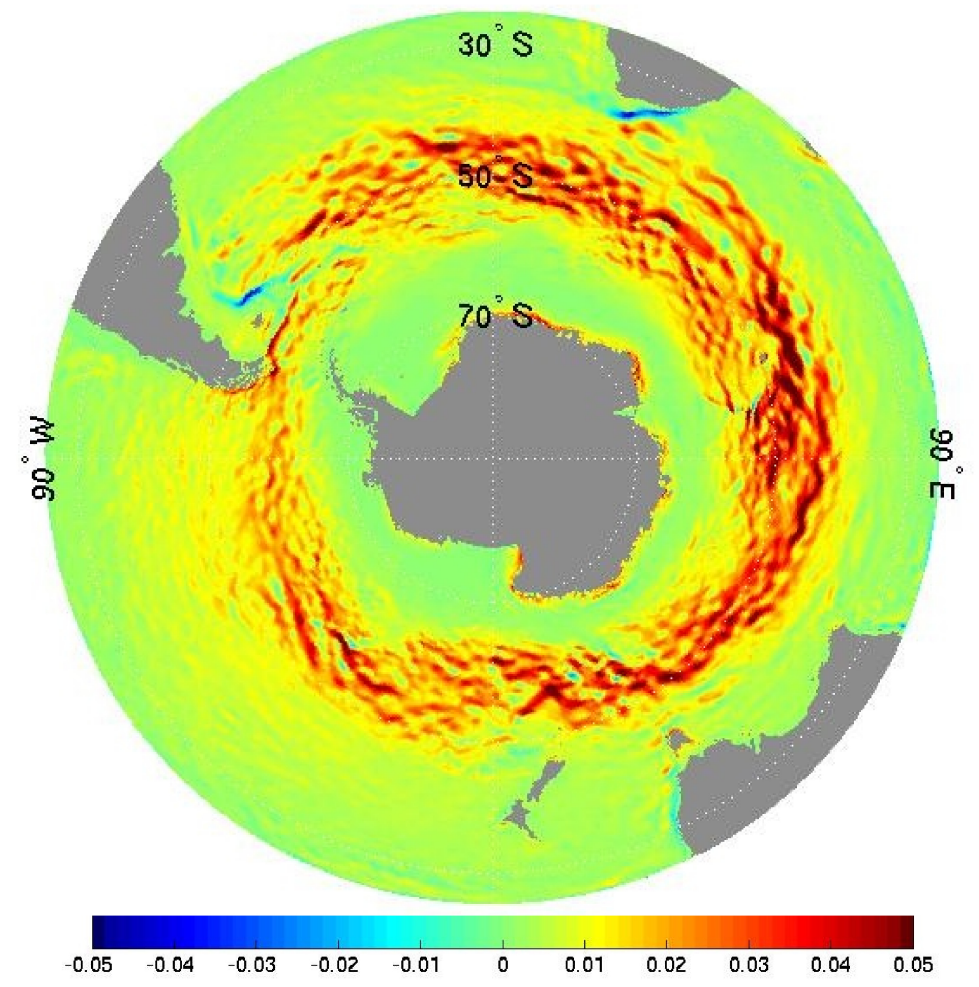

Figure 3-16: Time average rate of work (power) done by the wind on the Southern Ocean circulation, $\overline{\tau \cdot \mathbf{v}}$, in $\mathrm{W} / \mathrm{m}^{2}$. The integrated power input over the domain is $1.2 \mathrm{TW}$.

In the Southern Ocean state estimate, 0.5 TW is transferred to the ageostrophic flow, and the other $0.7 \mathrm{TW}$ to the geostrophic flow. Of these $1.2 \mathrm{TW}$ of wind power input to the Southern Ocean state estimate, over $0.3 \mathrm{TW}$, or almost 30\%, is transferred to high-frequency ocean fluctuations. ${ }^{3}$ While most of the wind work occurs in the ACC latitudes (see Figure 3-16), the transfer of energy to high-frequency oceanic fluctuations is not confined to the ACC region; it is large everywhere the wind is large. The power transferred to the global ocean's large-scale geostrophic currents occurs largely in the Southern Ocean. This region, however, is not such a dominant player when it comes to the power input to the other components of the oceanic circulation.

It has been proposed that a mechanism for dissipating eddy energy is through

\footnotetext{
${ }^{3}$ Here the so-called high-frequency ocean dynamics were those that could be removed with a 10 day running mean.
} 
wind stress working against eddy surface flows (Zhai and Greatbatch, 2007). This is an exciting area of research, because currently little is known about the dissipation of eddy energy. The theory states that when eddies are spinning in smooth wind fields, a stronger relative velocity between the atmosphere and ocean is present when the eddy flow counters the wind. This usually translates to a wind stress spinning down the eddy that is larger than the wind stress acting to spin up the eddy. Support is drawn from the fact that small-scale ocean processes do have a clear influence on the QuickSCAT scatterometer observations of wind stress (Chelton et al., 2004). Using an eddy permitting ocean model, Zhai and Greatbatch (2007) find a 10\% decrease in eddy kinetic energy over the North Atlantic when they take ocean surface velocities into account in the calculation of wind stress via bulk formulae. They also find that accounting for the relative velocity results in a $17 \%$ decrease in total rate of wind work over the North Atlantic.

One might expect this phenomenon to result in a similar decrease in the rate of wind work over the eddy-rich Southern Ocean (Hughes and Wilson, 2007). In the state estimate produced, however, the wind stress calculated only takes into account the wind velocity, and not the ocean surface velocity. Because the relative velocity was not accounted for, this mechanism for reducing the rate of wind work and spinning down the eddies is not present. For this reason it is possible that the calculated wind power input given above for both the ECCO-GODAE state estimate and this Southern Ocean state estimate is an upper bound. In the Southern Ocean, however, the wind velocities are significantly larger than the ocean velocities, and it is questionable how big an effect the ocean currents will have on the relative velocity. Nevertheless, future work should include the ocean surface velocities when calculating wind stress via bulk formulae.

All the analyses discussed in this chapter, including the wind work analysis above, are carried out on iteration 26. The mean kinetic energy of this iteration was found to be $5 \%$ less than that of the iteration 0 , and it is likely that the lower mean kinetic energy value results largely from control adjustments to the wind. It is not clear whether the spin-down occurs on the eddy or mean flow. Why the optimization has 
reduced the solutions kinetic energy should be investigated in future work.

\subsection{Conclusions}

Chapter 2 showed that the state estimate is largely consistent with the observations. Here we have found that the integrated freshwater, temperature, and volume transports in the state estimate are, for the most part, consistent with the recent static inverse estimates. No gross inconsistencies have been found. There are no revelations to be made with regards to heating or freshening of the Southern Ocean. This may be interpreted, however, as a promising conclusion. What has been accomplished, in a sense, is an improvement to static inverse models through both increased resolution and the addition of realistic dynamics. This is useful as it allows one to carry out, with confidence, detailed analyses (for example on the formation and evolution of water masses or on the dynamical balances) in the highly-energetic and eddy-rich Southern Ocean. 


\section{Chapter 4}

\section{Dynamics of the Meridional Overturning Circulation}

The Southern Ocean ventilates large regions of the World Oceans, with its signatures found even in the North Atlantic intermediate waters (Marsh et al., 2000). This role in ventilation makes the Southern Ocean important in determining the global climate. To quantify and understand the Southern Ocean's function in climate, it is necessary to determine its circulation. One wishes to understand the Antarctic Circumpolar Current (ACC) system, and the meridional overturning circulation (MOC). Of course the two are fundamentally linked as the shear of the Antarctic Circumpolar Current is coupled to the overturning circulation through the meridional density structure.

The following section provides an overview of the theories describing the Southern Ocean circulation. These theories assume simple physical balances to diagnose the structure of the ACC and MOC. In Section (4.2) the Southern Ocean state estimate is used to analyze the zonal momentum balance in isopycnal coordinates. This analysis illuminates the force balance of the MOC, and in doing so tests assumptions made in the theories described in Section (4.1). It is found that theories of the MOC must include the effect of zonal pressure forces. 


\subsection{Theoretical Review}

\subsubsection{Sverdrupian Theories: A Southern Ocean Gyre}

The lack of a complete meridional boundary has proven problematic in theorizing about the Southern Ocean. Theories regarding the circulation of the other major ocean basins are usually based on an interior in Sverdrup balance ${ }^{1}$ where the vorticity imparted on a fluid column by the wind drives the columns movement in the planetary vorticity gradient. A western boundary closes the mass balance while also closing the vorticity budget through frictional dissipation against the bottom and side-walls. For more details on Sverdrupian theories of oceanic circulations see Pedlosky (1996). Section 1.4 of this reference notes, however, that there is reason to doubt the relevance of Sverdrup balance in the actual ocean. It is especially hard to rationalize how theories based on Sverdrup balance could be used to explain the Southern Ocean circulation since the Drake Passage creates a discontinuity in the western boundary.

Stommel (1957) noted that while the opening in Drake Passage is 2000m deep, there are topographic barriers at other longitudes over most of its span. He assumed that sufficient boundary layer flows could be set up to allow for a complete western boundary current, and thus attempted to explain the Antarctic Circumpolar Current (ACC) using Sverdrup balance. Stommel's sketch of how this would work has since been elaborated on (e.g. Webb (1993)), and lent support by its general consistency with the observed Southern Ocean (Warren et al., 1996). The study of Warren et. al. (1996) noted that the meridional deviation of the current is consistent with what Sverdrup balance dictates under the observed wind forcing. The Falkland (or Malvinas) Current, which flows along the eastern boundary of South America, is thought to be the primary western boundary current, and Warren et. al. cited references showing that integrating the wind stress curl over the latitudes of this current does predict a Drake Passage transport consistent with observations. The error bars on both the wind forcing and observed transport were, however, quite large. Furthermore, no

\footnotetext{
${ }^{1}$ The Sverdrup balance is $\beta V_{S}=\hat{k} \cdot \nabla \times\left(\vec{\tau} / \rho_{0}\right)$, where $\beta$ is the northward gradient of the Coriolis parameter, $V_{S}=\int_{-H}^{0} v d z$ is the vertical integral of the meridional velocity, $\hat{k}$ is the unit vector in the vertical direction, $\tau$ is the wind stress, and $\rho_{0}$ is the mean density.
} 
mechanism was given as to how the current can cross the unblocked Drake Passage latitudes.

Though one can argue that most latitudes are in fact blocked, the models based on an interior Sverdrup balance and side-wall frictional boundary layers do become incomplete if any latitudes are unblocked. A theory that does allow for unblocked latitudes was derived by Gill (1968), who employed a rectangular basin geometry with an opening in the meridional wall to represent the Drake Passage. A boundary condition of periodicity was applied at the opening, and bottom friction was assumed to be the primary mechanism for vorticity dissipation. ${ }^{2}$ Having a mechanism to dissipate vorticity away from lateral boundaries frees the current to meander around the Drake Passage.

Gill divided the basin into different regions, and solved the vorticity equation subject to different assumptions in each one. In the regions of blocked latitudes standard gyre dynamics were assumed, i.e. an interior in Sverdrup balance with a western boundary layer region employed to close the mass and vorticity budget. A zonal channel flow was assumed in the unblocked latitudes. Boundary regions at the northern and southern limits of the unblocked latitudes were employed to satisfy momentum and pressure continuity requirements. These two open ocean boundary layer regions strongly coupled the circulations inside and outside of the circumpolar channel. It turns out, in fact, that instead of the circumpolar transport being governed by the channel dynamics that are dominant in the unblocked latitudes, the majority of the circumpolar transport resulted from frictional coupling with the currents outside the unblocked latitudes. Though the fluid inside the channel was treated as a zonal jet, a large proportion of it left the channel to enter into a western boundary current flow.

The theory is quite successful, in that it is able to reproduce the observed gyres in the Southern Ocean and the observed east-west asymmetry of the Antarctic Circumpolar Current. Gill showed the importance of remote forcing on the ACC. The current may be governed by channel dynamics, but a theory of its transport will be

\footnotetext{
${ }^{2} \mathrm{~A}$ discussion of the impact lateral friction would have on the results was included in the work.
} 
incomplete unless one accounts for its interactions with the subtropical and polar gyres abutting it. Perhaps most importantly, Gill found that one cannot decipher the circulation of the Southern Ocean by analyzing the vorticity budget alone. Solving for the vertically integrated transport streamfunction using the vorticity equation requires that the streamfunction be set to zero on one side of the unblocked latitudes, and equal to an undetermined constant, $\Psi_{t o t}$, on the the other side. The zonal transport through Drake Passage is determined by the magnitude of $\Psi_{\text {tot }}$. To determine $\Psi_{t o t}$, and in doing so find a unique solution to the model, one must ensure pressure is continuous in the domain. This constraint is lost with the elimination of pressure in the derivation of the vorticity equation. The problem is not fully posed if considering only the vorticity budget, and this forces one to look beyond simple gyre theories for explaining the Southern Ocean circulation.

\subsubsection{Zonal Channel Theories}

Gill (1968) found that zonal channel dynamics, and thus the zonal momentum balance, must be considered in the unblocked latitudes of the Southern Ocean. An issue arises, however, when considering these dynamics in an homogenous flat bottom model ocean. Zonal winds over the Southern Ocean drive an equatorward transport at the surface, and this mass balance must be closed. The net zonal pressure gradient, and thus the net meridional geostrophic transport vanishes in this model configuration. The poleward transport must be balanced by a bottom Ekman layer. Unfortunately, using a reasonably sized diffusivity requires an unreasonable zonal velocity to sustain the layer. This has become known as "Hidaka's dilemma" (Olbers et al., 2004). Hidaka's dilemma can be broken, however, by implementing variable ocean topography.

Variable ocean topography can decouple the zonal transport from the zonal momentum equation by allowing the mass budget to be closed via a net geostrophic transport. This can be seen from the zonally and vertically integrated steady-state 
zonal momentum equation:

$$
\begin{aligned}
-f \oint \int_{-D}^{0} v d z d x & =\oint \int_{-D}^{0}\left(-p_{x}+\mathcal{F}_{z}\right) d z d x \\
0 & =\oint \int_{-D}^{0}-p_{x} d z d x+\oint \mathcal{F}_{\text {surface }} d x-\oint \mathcal{F}_{\text {bottom }} d x
\end{aligned}
$$

Here $f=2 \Omega \sin (\phi)$ is the Coriolis parameter $(\Omega$ being the angular velocity of the earth and $\phi$ being latitude), $D$ is the total fluid depth, $v$ is the meridional velocity, $p_{x}$ is the zonal pressure gradient, and $\mathcal{F}$ is the frictional stress exerted on the fluid. Nonlinear advection, interior momentum dissipation, and horizontal diffusion have been neglected (i.e. a small Ekman and Rossby number have been assumed) as these terms are believed to be higher order in the balance of Equation (4.1) (Rintoul et al., 2001). Note that $\oint \int_{-D}^{0} v d z d x$ is required to vanish by mass conservation (assuming outflow from Antarctica is negligible). At the surface, $\mathcal{F}$ is dominated by the atmospheric winds, and at the bottom it is a function of the ocean velocity field. As stated above, the ACC is not strong enough to allow bottom friction to come into the lowest order time-mean balance (Olbers et al., 2004). The presence of topography at depth, however, can support zonal pressure gradients, and thus bottom Ekman layers are not needed. It has been established in various modeling studies (Rintoul et al., 2001), and is also found in the Southern Ocean state estimate, that Equation (4.1) is, to lowest order,

$$
\oint \mathcal{F}_{\text {surface }} d x=\oint p_{x} d x \approx \sum_{\text {ridges }} \delta p_{\text {bottom }}
$$

This shows that the vertically integrated momentum budget is maintained by an inward flux of momentum at the surface being balanced by a removal via topographic interactions.

The constraint of Equation (4.2) renders the idealized model of Gill (1968) well posed, while providing a satisfactory way to close the mass balance. Nevertheless, vertically integrated models of this nature do not produce a full description of the Southern Ocean circulation. One wishes to know the vertical structure, and it is 
apparent that the baroclinicity of this structure is fundamental. In fact, assuming the Southern Ocean is homogenous makes it impossible to explain the ACC. This is demonstrated nicely with three numerical calculations carried out by Olbers and Eden (2003). The three Southern Ocean models they investigated all had the same wind stress applied at the surface, and thus they all had similar Ekman transports. The first experiment had an homogenous ocean and a flat bottom. As explained above, the only way mass (and vorticity) could be balanced on circumpolar streamlines was through a frictional bottom boundary layer. In this scenario the transport of the Antarctic Circumpolar Current became much larger than observed (over $600 \mathrm{~Sv}$ ) so that this bottom boundary layer could be effective. (The dissipation parameterization used in this model was a linear drag. Using a higher order drag or a larger drag coefficient would reduce the transport. Thus the magnitude of the transport is rather irrelevant; what's important is the conclusion that for this set-up, a reasonable representation of turbulent bottom boundary layer processes leads to unreasonably large ACC transports.) In the second model Olbers and Eden introduced more realistic bathymetry. Now the meridional Ekman flux could be more easily balanced by deep western boundary currents. In this regime, where topographic stretching of the water column becomes important, the Antarctic Circumpolar Current transport dropped to approximately $30 \mathrm{~Sv}$, a value much smaller than observed. In Olbers and Eden's last experiment they added a density structure, and, as a result, a reasonable Antarctic Circumpolar Current transport was found. The conclusion is that the baroclinicity of the Southern Ocean is a key factor in determining the zonal transport. Vortex stretching within the water column plays a significant role in the Antarctic Circumpolar Current dynamics, as it allows the current to deviate from $f / D$ contours (where $D$ is the ocean depth).

Olbers and Eden (2003), and others (e.g. Gnanadesikan and Hallberg (2000)), have shown the importance that stratification has on the Southern Ocean circulation. A simple theory was sketched by Samelson (1999) in attempt to explain the observed stratification. The geometry was the same as in Gill (1968), except that the gap did not extend to the basin floor and the meridional boundary was extended to account 
for a northern hemisphere. Samelson concluded that because the waters formed in the southern polar gyres are the densest in the oceans, they should sink to the sill depth of the circumpolar channel, and then spread northward to fill the abyssal ocean. Above the sill depth geostrophic constraints keep this dense water from crossing the gap. At the surface a northward Ekman transport drives the dense polar waters across the channel. As the Ekman driven surface waters cross the gap, they gain buoyancy such that when they reach the equatorward edge of the sill they are less dense than waters formed in the polar gyres of the northern hemisphere. These Ekman driven surface waters then subduct to ventilate the subtropical thermocline and eventually mix upwards. The northern hemisphere deep waters are able to fill the oceans from the sill depth to the depths of the wind driven thermoclines. This deep water will be the water that flows along the sill into the southern hemisphere polar oceans to balance the mass lost from the Ekman transport. To some degree this is what is observed, as North Atlantic Deep Water flows into the Ross and Weddell Seas at approximately the Drake Passage sill depth.

In the model of Samelson (1999), the shear of the Antarctic Circumpolar Current is set by the density gradient across the Drake Passage gap, and therefore by the location and magnitude of the deep water formation. Various studies have elaborated on Samelson (1999), some of which have used numerical models (Vallis, 2000; Samelson, 2004; Fučkar and Vallis, 2007). The models highlight the controlling effect that the circumpolar channel has on the inter-hemispheric structure of the meridional overturning circulation. Modeling studies note, however, that eddies allow this controlling effect to be diminished. It has become apparent that the role of eddies in the Southern Ocean dynamics warrants further investigation (Wolfe and Cessi, 2008).

Consideration of the buoyancy budget emphasizes the important role that eddies are believed to play in the Southern Ocean circulation (Kuo et al., 2005). In models of the nature of Samelson (1999), an equatorward surface mass transport is balanced by a poleward mass transport at depth. This requires a buoyancy loss equatorward of the ACC that allows fluid to downwell, and also a buoyancy gain in the ACC latitudes where fluid is upwelling (Kuo et al., 2005). The Southern Ocean buoyancy budget 
shows that there is a buoyancy loss in the ACC latitudes (Cerovecki et al., 2008), and therefore, since the ocean is stably stratified, a net equatorward mass transport at the surface is highly improbable. How could water upwell to satisfy this Ekman pumping in the presence of a buoyancy loss? The near surface equatorward Ekman transport must be overshadowed by another mass transport mechanism. If the ACC is described as an unblocked channel then there is no net geostrophic flow since the zonal pressure gradient integrates to zero. Furthermore, the shear of the Antarctic Circumpolar Current is not nearly large enough to drive significant viscous flows. Neglecting pressure and viscous forcing leaves eddy forcing as the most probable mechanism to balance the Ekman transport.

When pressure gradients are integrated between material surfaces (e.g. between isopycnals) boundary contributions arise. Momentum may be redistributed through these interfacial boundary terms. When a transient eddy raises an isopycnal layer, a horizontal pressure gradient ( $p_{x}$ for the zonal direction) can push this anomaly ( $\eta^{\prime}$, where $\bar{\eta}=\eta-\eta^{\prime}$ is the mean isopycnal interface) and transfer momentum into the lower layer. As explained by Johnson and Bryden (1989), this so-called eddy interfacial form stress (IFS $\equiv \overline{\eta^{\prime} p_{x}^{\prime}}$ ) acts to redistribute momentum downwards, thus canceling the Ekman transport. Vertical momentum transport by IFS may diverge in the interior to release the momentum absorbed by the wind, or this momentum may be expelled at the ocean floor via pressure drops across topogrophy. Interfacial form stress provides a mechanism to close the buoyancy, mass, and momentum budgets locally and adiabatically.

\subsubsection{The Residual Meridional Overturning Circulation}

Interfacial form stress (IFS) may redistribute momentum vertically, layer by layer, from the surface to the ocean floor. There is, however, a depth dependent meridional overturning circulation (MOC) in the Southern Ocean, and thus the vertical momentum fluxes are diverging (Killworth and Nanneh, 1994). The idea of diagnosing a residual streamfunction, $\Psi^{*}$, was introduced by Andrews and McIntyre (1976) to 
understand the atmospheric circulation. This streamfunction can be written as

$$
\Psi^{*}=\bar{\Psi}+\hat{\Psi}
$$

where $\bar{\Psi}$ is the overturning circulation for the Eulerian mean flow, and $\hat{\Psi}$ is the circulation associated with eddy dynamics. The success of the residual formulation of Equation (4.3) to describe the interior stratosphere has prompted its application to the Southern Ocean meridional overturning circulation (e.g. Johnson and Bryden (1989); Marshall and Radko (2003); Olbers and Visbeck (2005)). In this context the surface Ekman flow and abyssal geostrophic or viscous flow is associated with $\bar{\Psi}$. The eddy form stress divergence drives the eddy streamfunction, $\hat{\Psi}$. At the surface an eddy stress may counter an equatorward wind-driven Ekman transport, and at depth a geostrophic transport may counter an equatorward eddy stress; the net transport, $\Psi^{*}$, will be a small residual of these larger opposing components.

Many studies have attempted to reconstruct the Southern Ocean residual circulation through consideration of IFS mechanisms (see Olbers et al. (2004) for a review). The first of these studies was Johnson and Bryden (1989) who determined the magnitude of the implied IFS by making the extreme assumption that the Southern Ocean is completely adiabatic (i.e. that there is no overturning circulation: $\Psi^{*}=0$ ). It follows that IFS is exactly balancing the zonal momentum input from the wind and only diverging at the ocean bottom where it is exactly balanced by pressure forces. Johnson and Bryden (1989) therefore assumed that $\bar{\Psi}=\hat{\Psi}$, or equivalently that $-f^{-1} \tau_{0}=\overline{\eta^{\prime} p_{x}^{\prime}} \approx f \overline{b^{\prime} v^{\prime}} \overline{N^{2}}-1$ where $\tau_{0}$ is the wind stress, $f$ is the Coriolis parameter, $b$ is buoyancy, $N$ is the buoyancy frequency, and geostrophy has been used to relate the form stress to the meridional transport of buoyancy. Using this relation, the observed wind forcing, and a horizontal down-gradient buoyancy flux parameterization $\left(\overline{b^{\prime} v^{\prime}} \approx-\kappa \overline{b_{y}}\right)$, Johnson and Bryden were able to determine the meridional density gradient to within the parameterization constant $\kappa$. The buoyancy gradient is related to the level of available potential energy, and therefore the level of baroclinic instability of the system. By suggesting that eddy activity is set through baroclinic 
instability processes, Johnson and Bryden set $\kappa$ proportional to the meridional buoyancy gradient $\left(\overline{b^{\prime} v^{\prime}} \approx-\kappa_{0}{\overline{b_{y}}}^{2}\right)$. Because thermal wind dictates that the meridional density gradient is proportional to the zonal velocity shear, they concluded that the shear in the Antarctic Circumpolar Current should be proportional to the square root of the zonal wind, $u_{z} \sim \tau_{0}^{1 / 2}$. This relationship has not been found in numerical models (Olbers et al., 2004), though Marshall and Radko (2003) argue that there is laboratory evidence for the validity of this scaling.

Numerical models often have success with parameterization schemes based on constant coefficients. Setting $\kappa$ constant for the Johnson and Bryden theory results in a linear scaling between the velocity shear and the wind stress, $u_{z} \sim \tau_{0}$. Similarly, however, this scaling has not been verified with numerical models (Olbers et al., 2004). It is not clear what is the best way to parameterize eddy buoyancy fluxes, and thus what the appropriate scaling should be. Furthermore, these theories are quite idealized (e.g. they assume a constant buoyancy frequency), and the validity of the a priori assumptions made is not certain. It is necessary to look to eddying numerical models for greater understanding of the residual circulation balance.

In the governing equations for the residual mean streamfunction (also referred to as the transformed Eulerian mean streamfunction), no "eddy" terms show up explicitly in the buoyancy equation; only diabatic processes force property transport across $\Psi^{*}$ streamlines. The residual circulation is, therefore, the flow that advects buoyancy and other water properties (e.g. CFCs), making it a better approximation of the Lagrangian mean circulation. For more on the formulation of the transformed Eulerian mean framework see Andrews et al. (1987); McIntosh and McDougall (1996); Lee and Coward (2003); Plumb and Ferrari (2005). Because buoyancy is advected by the residual mean streamfunction, one may reconstruct this circulation from knowledge of the buoyancy budget. Several theories have used this information to elaborate on the work of Johnson and Bryden (e.g. Marshall and Radko (2003, 2006); Olbers and Visbeck (2005); Ito and Marshall (2007)). The theories rely on atmospheric forcing to set both the surface buoyancy structure and the Ekman transport, making the residual streamfunction and its components known at the surface. They assume an 
adiabatic interior force balance, and propagate the surface conditions downward using this balance. The theories assume diapycnal mixing, and thus water mass formation, occurs only in the surface and bottom boundary layers of the ocean. Theories of this nature only work in the ventilated ocean regions, leaving the circulation in the abyss unknown. This thesis determines the structure of the full residual circulation. By doing so the assumptions made in the Johnson and Bryden (1989) type theories are tested. It is found that the relevance of the residual circulations inferred from these theories to the actual Southern Ocean is questionable.

The theories described above are formulated using depth as the vertical coordinate. Compared to analyses carried out in isopycnal space, the depth-space formulation can be advantageous near the surface where isopycnals outcrop. The depth-space formulation is, however, related to the volume weighted streamfunction in isopycnal space. This volume weighted residual streamfunction is defined as $\Psi^{*} \equiv \overline{v h}$, where $h$ is the layer thickness. (For a continuous system the layer thickness may be written in

terms of the buoyancy gradient, $h \equiv \rho_{0}\left(\rho_{z}\right)^{-1}$.) Owing to this rather simple definition of $\Psi^{*}$ theoretical formulations of the residual circulation in isopycnal space are often more straightforward (Plumb and Ferrari, 2005). In the following section the force balance for the residual circulation, $\Psi^{*}$, is calculated in isopycnal space.

\subsection{Derivation of the Zonal Momentum Balance in Isopycnal Space}

Addressing the details of the Southern Ocean residual circulation via the zonal momentum balance is the goal of this section. The calculation is carried out with the vertical coordinate being neutral density, $\gamma$, which largely removes the effects of compressibility from the in situ density (Jackett and McDougall, 1997). Derivation of the governing equations in isopycnal coordinates is carried out in various books (e.g. Vallis 2006, section 3.9) and papers (e.g. de Szoeke and Bennett (1993); Killworth and Nanneh (1994)). In this section the zonal momentum equation is manipulated to 
illuminate the force balance of the residual circulation. In Section (4.3) the leading order terms of the balance are presented in the form of components of the zonally integrated overturning streamfunction. As discussed in the previous section, current theories postulate that the residual circulation is the difference between an ageostrophic frictional (Ekman) stress and an eddy stress, with, perhaps, a geostrophic abyssal transport. Section (4.3) shows that there is a significant geostrophic contribution to the residual circulation at all depths due to blocked isopycnal contours. The effect that isopycnal outcropping has on the solution is highlighted. The buoyancy budget and a temporal drift in the state estimate is evaluated. A summary of the results is in Section (4.4).

When converting from $z$ coordinates to $\gamma$ coordinates the pressure gradient term becomes

$$
\begin{aligned}
\partial p /\left.\partial x\right|_{z} & =\partial p /\left.\partial x\right|_{\gamma}-\partial z /\left.\partial x\right|_{\gamma} \partial p / \partial z \\
& =\partial p /\left.\partial x\right|_{\gamma}+g \rho \partial z /\left.\partial x\right|_{\gamma}
\end{aligned}
$$

where the hydrostatic relation, $p_{z}=-g \rho$, has been used. Here $g$ is the gravitational acceleration, and $\rho$ is the density. Accordingly, the zonal momentum equation in $z$ space,

$$
D u / D t-f v=\left(1 / \rho_{0}\right)\left(-p_{x}+F\right)
$$

becomes in $\gamma$-space

$$
\begin{aligned}
D u / D t-f v & =\left(1 / \rho_{0}\right)\left(-p_{x}-g \rho z_{x}+F\right) \\
& =\left(1 / \rho_{0}\right)\left(-p_{x}-g \gamma z_{x}-g(\rho-\gamma) z_{x}+F\right) \\
& =\left(1 / \rho_{0}\right)\left(-(p+g z \gamma)_{x}-g(\rho-\gamma) z_{x}+F\right) \\
& =\left(1 / \rho_{0}\right)\left(-M_{x}-g(\rho-\gamma) z_{x}+F\right) .
\end{aligned}
$$


The Montgomery potential,

$$
M \equiv p+g z \gamma
$$

has been introduced in Equation (4.6). The term $g(\rho-\gamma) z_{x}$ arises from differences between $\rho$ and $\gamma$ surfaces caused by compressive effects.

For this calculation the material derivative is written as

$$
D u / D t=\partial u / \partial t+(1 / 2)\left(u^{2}+v^{2}\right)_{x}-\zeta v+Q u_{\gamma}
$$

where $\zeta \equiv v_{x}-u_{y}$ is the relative vorticity of the fluid. Here $Q \equiv \gamma_{0}^{-1} D \gamma / D t$ is the density tendency. It is important to remember that this is the material derivative in $\gamma$-space, and thus all "horizontal" derivatives are taken along $\gamma$ surfaces. The zonal momentum equation (4.5) becomes

$$
\left(\partial u / \partial t+(1 / 2)\left(u^{2}+v^{2}\right)_{x}+Q u_{\gamma}-(f+\zeta) v\right) \mathcal{T}=\left(1 / \rho_{0}\right)\left(-M_{x}-g(\rho-\gamma) z_{x}+F\right) \mathcal{T}
$$

The top hat function,

$$
\mathcal{T} \equiv \mathcal{H}\left(\gamma-\gamma_{s}\right)-\mathcal{H}\left(\gamma-\gamma_{b}\right)
$$

where $\mathcal{H}$ is the Heaviside function, is employed to ensure the forcing vanishes outside of the ocean $\left(\gamma_{s}(x, y, t)\right.$ and $\gamma_{b}(x, y, t)$ are the density values at the surface and bottom of the ocean).

The potential vorticity is defined as $P \equiv(f+\zeta) / h$, where $h$ is the layer thickness. For a continuous system the thickness takes the form of $h \equiv \rho_{0} \rho_{z}^{-1}$, such that $P$ becomes equivalent to the Ertel potential vorticity. Using $P$, Equation (4.9) becomes

$$
\left(u_{t}+(1 / 2)\left(u^{2}+v^{2}\right)_{x}+Q u_{\gamma}-h P v\right) \mathcal{T}=\left(1 / \rho_{0}\right)\left[-M_{x}-g(\rho-\gamma) z_{x}+F\right] \mathcal{T}
$$


Taking a temporal mean, which is denoted by an overbar, yields

$$
\begin{aligned}
\overline{u_{t} \mathcal{T}}+(1 / 2)\left(\overline{\left(u^{2}\right)_{x} \mathcal{T}}+\overline{\left(v^{2}\right)_{x} \mathcal{T}}\right) & +\overline{Q u_{\gamma} \mathcal{T}}-\overline{h P v \mathcal{T}} \\
& =\left(1 / \rho_{0}\right)\left[-\overline{M_{x} \mathcal{T}}-\overline{g(\rho-\gamma) z_{x} \mathcal{T}}+\overline{F \mathcal{T}}\right]
\end{aligned}
$$

Dividing by the mean thickness, $\bar{h}$ and denoting a thickness weighted mean as $\bar{A}^{*}=$ $\overline{A h} / \bar{h}$ this becomes

$$
\begin{aligned}
\left(\overline{u_{t} \mathcal{T}}+(1 / 2)\left(\overline{\left(u^{2}\right)_{x} \mathcal{T}}+\overline{\left(v^{2}\right)_{x} \mathcal{T}}\right)\right. & \left.+\overline{Q u_{\gamma} \mathcal{T}}\right) \bar{h}^{-1}-{\overline{P v \mathcal{T}^{*}}}^{*} \\
& =\left(\rho_{0} \bar{h}\right)^{-1}\left[-\overline{M_{x} \mathcal{T}}-\overline{g(\rho-\gamma) z_{x} \mathcal{T}}+\overline{F \mathcal{T}}\right] .
\end{aligned}
$$

Denoting the departure from the volume weighted mean with a hat, $(\hat{\bullet})$, one may define $P \equiv \bar{P}^{*}+\hat{P}$ and $v \equiv \bar{v}^{*}+\hat{v}$. Neglecting cross terms (i.e. $\overline{\bar{P}}^{*} \hat{v}=\overline{\hat{P}}^{*}{ }^{*}=0$ ) the Reynolds decomposition becomes

$$
\overline{P v \mathcal{T}}^{*} \equiv \overline{P \mathcal{T}}^{*} \overline{v \mathcal{T}}^{*}+\overline{\hat{P}} \hat{v} \mathcal{T}^{*}
$$

(note $\mathcal{T}$ is either 0 or 1 , such that $\mathcal{T}^{2}=\mathcal{T}$ ) and Equation (4.13) becomes

$$
\begin{aligned}
\left(\overline{u_{t} \mathcal{T}}+(1 / 2)\left(\overline{\left(u^{2}\right)_{x} \mathcal{T}}+\overline{\left(v^{2}\right)_{x} \mathcal{T}}\right)\right. & \left.+\overline{Q u_{\gamma} \mathcal{T}}\right) \bar{h}^{-1}-\overline{P \mathcal{T}}^{*} \overline{v \mathcal{T}}^{*}-\overline{\hat{P}} \hat{v} \mathcal{T}^{*} \\
& =\left(\rho_{0} \bar{h}\right)^{-1}\left[-\overline{M_{x} \mathcal{T}}-\overline{g(\rho-\gamma) z_{x} \mathcal{T}}+\overline{F \mathcal{T}}\right]
\end{aligned}
$$

The thickness averaged circulation is thus given by

$$
\begin{aligned}
\overline{v \mathcal{T}}^{*}= & \left(\bar{h} \overline{P \mathcal{T}}^{*}\right)^{-1}\left(\overline{u_{t} \mathcal{T}}+(1 / 2)\left(\overline{\left(u^{2}\right)_{x} \mathcal{T}}+\overline{\left(v^{2}\right)_{x} \mathcal{T}}\right)+\overline{Q u_{\gamma} \mathcal{T}}\right)-\overline{P \mathcal{T}}^{*-1} \overline{\hat{P}} \hat{v} \mathcal{T}^{*} \\
& +\left(\rho_{0} \bar{h} \overline{P \mathcal{T}}^{*}\right)^{-1}\left[\overline{M_{x} \mathcal{T}}+\overline{g(\rho-\gamma) z_{x} \mathcal{T}}-\overline{F \mathcal{T}}\right]
\end{aligned}
$$

This equation is analogous to Equation 6 in Schneider (2005), and similarly to what was done in that work, the pressure forcing contributions are decomposed below in order to shed light on various dynamical processes. The derivative of the top hat function is two Dirac delta functions, $\mathcal{T}_{x}=-\gamma_{s_{x}} \delta\left(\gamma-\gamma_{s}\right)+\gamma_{b_{x}} \delta\left(\gamma-\gamma_{b}\right)$, and using 
this fact the Montgomery potential may be split as follows:

$$
\begin{aligned}
\overline{M_{x} \mathcal{T}}= & (\overline{M \mathcal{T}})_{x}-\overline{M \mathcal{T}_{x}} \\
= & (\overline{M \mathcal{T}})_{x}+\overline{M \gamma_{s_{x}} \delta\left(\gamma-\gamma_{s}\right)}-\overline{M \gamma_{b_{x}} \delta\left(\gamma-\gamma_{b}\right)} \\
= & \left(\overline{M \mathcal{T}}+\overline{M \gamma_{s} \delta\left(\gamma-\gamma_{s}\right)}-\overline{M \gamma_{b} \delta\left(\gamma-\gamma_{b}\right)}\right)_{x} \\
& -\overline{M_{x} \gamma_{s} \delta\left(\gamma-\gamma_{s}\right)}+\overline{M_{x} \gamma_{b} \delta\left(\gamma-\gamma_{b}\right)} \\
= & \left(\overline{M \mathcal{T}}+\overline{M \gamma_{s} \delta\left(\gamma-\gamma_{s}\right)}-\overline{M \gamma_{b} \delta\left(\gamma-\gamma_{b}\right)}\right)_{x} \\
& +f \rho_{0}\left(-\overline{v_{g} \gamma_{s} \delta\left(\gamma-\gamma_{s}\right)}+\overline{v_{g} \gamma_{b} \delta\left(\gamma-\gamma_{b}\right)}\right) .
\end{aligned}
$$

The interior pressure forcing takes the form of term (4.17). In the absence of lateral boundaries this body force will vanish by periodicity. When isopycnals outcrop zonally at either the ocean floor or surface (i.e. zonal density gradients exist at these boundaries), a pressure force at the boundary can be projected into the interior. This effect, which is related to bottom and surface form drag, has been represented as a density flux (terms 4.18) using the geostrophic relationship, $M_{x}=f \rho_{0} v_{g}$, where $v_{g}$ is the geostrophic velocity. This term demonstrates that a surface buoyancy flux can balance a volume weighted mean flow.

When solving for the mean volume weighted flow, it is practical to multiply by the mean thickness such that upon zonal (or streamwise) integration the units become a transport in Sverdrups. The force balance to be solved becomes

$$
\begin{aligned}
\bar{h} \overline{v \mathcal{T}}^{*}=\left(\overline{P \mathcal{T}^{*}}\right)^{-1} & {\left[\overline{u_{t} \mathcal{T}}+(1 / 2)\left(\overline{\left(u^{2}\right)_{x} \mathcal{T}}+\overline{\left(v^{2}\right)_{x} \mathcal{T}}\right)+\overline{Q u_{\gamma} \mathcal{T}}-\bar{h} \overline{\hat{P} \hat{v} \mathcal{T}}\right.}
\end{aligned}
$$

Using the Southern Ocean state estimate the various terms in this equation are evaluated in the next section. 


\subsection{Results}

The Southern Ocean zonal momentum equation (4.19) is evaluated in this section to determine the force balance of the meridional overturning circulation (MOC). Terms that have been found to be higher order, and have thus been neglected, are discussed in subsection (4.3.1). Difficulties in calculating individual pressure forcing contributions are also discussed in (4.3.1). Subsections (4.3.2), (4.3.3), and (4.3.4) then discuss the ageostrophically balanced circulation, geostrophically balanced circulation, and total circulation respectively. These circulations are presented in terms of a zonally integrated MOC streamfunction. The remainder of the section analyzes certain aspects of these circulations, including the advective buoyancy flux (4.3.5), the effect of drifts in the solution (4.3.6), and the eddy components (4.3.7) and (4.3.8).

\subsubsection{Introduction}

There is a substantial seasonal cycle in both the strength of the atmospheric winds and the Southern Ocean's mixed layer depth. Because the mixed layer depth is linked to the wind strength, correlations between wind driven Ekman transport and the surface potential vorticity can be considerable. Equation (4.19) has been evaluated seasonally in an attempt to remove the seasonal cycle from the eddy potential vorticity flux. The seasons chosen are December to February (Austral summer), March to May (fall), June to August (winter), and September to November (spring). The terms in Equation (4.19) are time-averaged over each season for the two year (2005-2006) state estimate.

The zonal acceleration term, $u_{t}$, is found to balance an order $0.1 \mathrm{~Sv}$ transport, and is thus negligible. The seasonal differences in the residual circulation are significant, however, suggesting that the seasonal state equilibrates on time-scales shorter than the approximately ninety day seasons. The order-Rossby number kinetic energy term, $(1 / 2)\left(u^{2}+v^{2}\right)_{x}$, is also negligible. Similarly, the vertical momentum thickness flux term, $Q u_{\gamma}$, has been found to balance an order $0.1 \mathrm{~Sv}$ transport and is thus negligible in the lowest order budget. Therefore, to lowest order (perhaps with an error of 
approximately 1\%), Equation (4.19) becomes

$$
\begin{aligned}
\bar{h} \bar{v}^{*}=\left(\bar{P}^{*}\right)^{-1} & {\left[-\bar{h} \overline{\hat{P} \hat{v}}-\rho_{0}^{-1} \bar{F}\right.} \\
& -\rho_{0}^{-1}\left(\bar{M}+\overline{M \gamma_{s} \delta\left(\gamma-\gamma_{s}\right)}-\overline{M \gamma_{b} \delta\left(\gamma-\gamma_{b}\right)}\right)_{x} \\
& \left.+f\left(\overline{v_{g} \gamma_{s} \delta\left(\gamma-\gamma_{s}\right)}-\overline{v_{g} \gamma_{b} \delta\left(\gamma-\gamma_{b}\right)}\right)-\rho_{0}^{-1} \overline{g(\rho-\gamma) z_{x}}\right] .
\end{aligned}
$$

Neutral density is a label for a locally referenced potential density, it is not an intrinsic variable (Jackett and McDougall, 1997). Contours of neutral density are continuous, and because fluid particles flow adiabatically along them, these contours are useful paths of integration. The reference pressure used to calculate neutral density is only locally consistent; it changes along the integration path. When converting from in situ density, $\rho$, to neutral density, $\gamma$, it is therefore not clear how the in - situ reference density, $\rho_{0}$, maps to $\gamma_{0}$. The full pressure forcing can be calculated in $z$ space and mapped to $\gamma$-space without error, and thus the total pressure contribution may be determined. It is not trivial, however, to calculate the individual pressure forcing contributions that arise in Equation (4.20), and these calculations are left for future work. The pressure contribution associated with buoyancy fluxes, $\bar{P}^{*-1} f \overline{v_{g} \gamma_{s} \delta\left(\gamma-\gamma_{s}\right)}$, is worth speculating on, however, as it is not associated with topographic interactions and therefore rather unfamiliar. As a first approximation of this term's structure the assumption was made that compressive effects are negligible near the surface such that $\gamma_{s} \approx \rho_{s}$. This assumption, which leads to the relation $\overline{\left.v_{g}\right|_{\gamma} \gamma_{s} \delta\left(\gamma-\gamma_{s}\right)} \approx \overline{\left.v_{g}\right|_{z} \rho_{s} \delta\left(\gamma-\gamma_{s}\right)}$, may introduce significant errors where isopycnal slopes are steep in the zonal direction.

The mean potential vorticity, $\bar{P}^{*}$, is defined as $\overline{(f+\zeta) h} / \bar{h}$. As the planetary vorticity, $f$, is only a function of latitude the mean $\mathrm{PV}$ can be written as $\bar{P}^{*}=$ $f \bar{h} / \bar{h}+\overline{\zeta h} / \bar{h}$ for either temporal or zonal integration. $\zeta$ will vanish where isopycnal layers vanish, but the planetary vorticity will not. A conflict arises as to what to do when isopycnals, and thus $\bar{h}$, vanishes. When this occurs one could either set $\bar{P}^{*}=f$, as in Schneider (2005), or $\bar{P}^{*}=0$, as in Koh and Plumb (2004). The advantages of each are discussed in detail in Schneider (2005). For the temporal averaging in this 
work the convention of Koh and Plumb (2004) was used (convention II as discussed in Schneider (2005)), such that $\bar{P}^{*}=0$ where isopycnals vanish. As zonal integration was carried out on full terms (e.g. $\bar{h} \overline{\hat{P}} \hat{v}^{*}\left(\bar{P}^{*}\right)^{-1}$ as a whole, as oppose to on both $\bar{h} \overline{\hat{P}}^{*}$ and $\left(\bar{P}^{*}\right)^{-1}$ independently) this choice does not effect the zonal integral as velocity and forcing terms vanish when isopycnals vanish. The choice made to set $\bar{P}^{*}=0$ when isopycnals outcrop in time results in a smaller value for the mean potential vorticity in near surface layers with frequent temporal outcrops. It is likely that the force balance would not change qualitatively had the other convention (i.e. $\bar{P}^{*}=f$ when $\left.\bar{h}=0\right)$ been used.
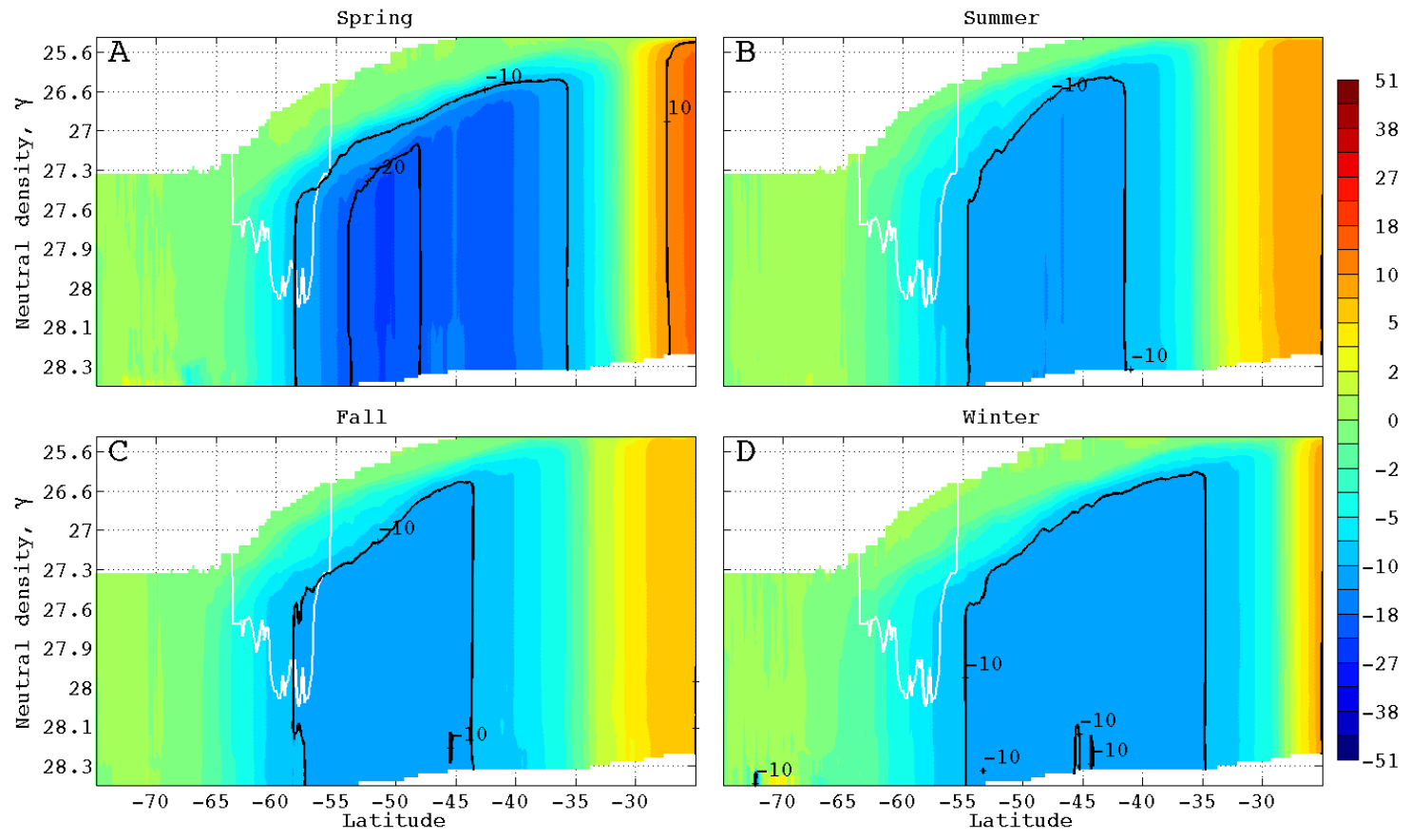

Figure 4-1: The transport streamfunction balanced by mean wind and viscous forcing in $\mathrm{Sv}$ (term $-\left(\rho_{0} \bar{P}^{*}\right)^{-1} \bar{F}$ of Equation 4.20). Positive (red) values denote counterclockwise flows, and negative (blue) values denote clockwise flows. The circulation has been averaged over the Austral seasons: spring (September to November) in Panel A, summer (December to February) in Panel B, fall (March to May) in Panel C, and winter(June to August) in Panel D. The white line shows the approximate location of latitudes that are not blocked by bottom topography. Note the ordinate is stretched in $\gamma$-space by a factor that reflects the volume of water at each density class. The color axis is also stretched. 

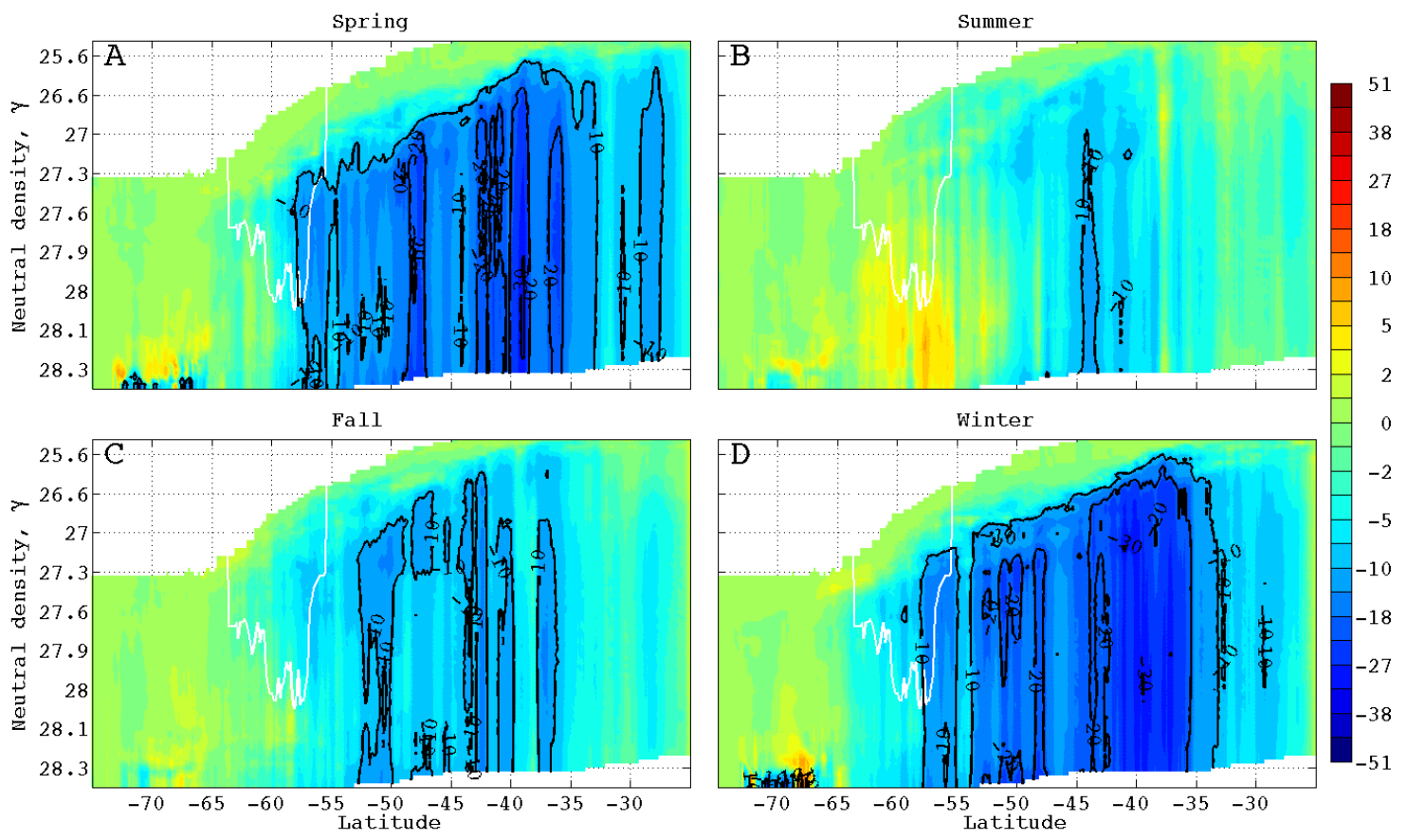

Figure 4-2: The transport streamfunction balanced by forcing from eddy potential vorticity fluxes $(\mathrm{PV})$ in $\mathrm{Sv}$ (term $-\bar{P}^{*-1} \bar{h} \overline{\hat{P}}^{*}$ of Equation 4.20). The residual circulation balanced by eddy PV fluxes does not oppose the Ekman transport, and this is discussed in Section (4.3.8). This figure is comparable to Figure (4-1). The good assumption that $P \approx f h^{-1}$ makes this term equivalent to $\overline{v^{\prime} h^{\prime}}$, and thus one may compare this figure to the two year mean $\overline{v^{\prime} h^{\prime}}$ streamfunction plotted in Figure $(3-13)$.

\subsubsection{The Ageostropohic Residual Circulation}

Mechanical forcing balances an approximately $10 \mathrm{~Sv}$ flow in the lightest water classes over most the year (Figure 4-1). This forcing is a result of wind driven Ekman transport; the effect of viscous forces is higher order. The forcing is strongest in the spring when it balances a flow greater than $20 \mathrm{~Sv}$. The wind field has a significant divergence in the unblocked latitudes, and a strong convergence equatorward of $40^{\circ} \mathrm{S}$.

The wind driven Ekman flow averages approximately $30 \mathrm{~Sv}$ (see Section 3.2.3). This transport is not all accounted for by the seasonal mean wind forcing in the surface layer; much of the Ekman transport is accomplished through a wind-driven eddy potential vorticity (PV) flux. The eddy PV flux balances an equatorward flow occurring in the lightest density classes (Figure 4-2). The flow ranges from approxi- 

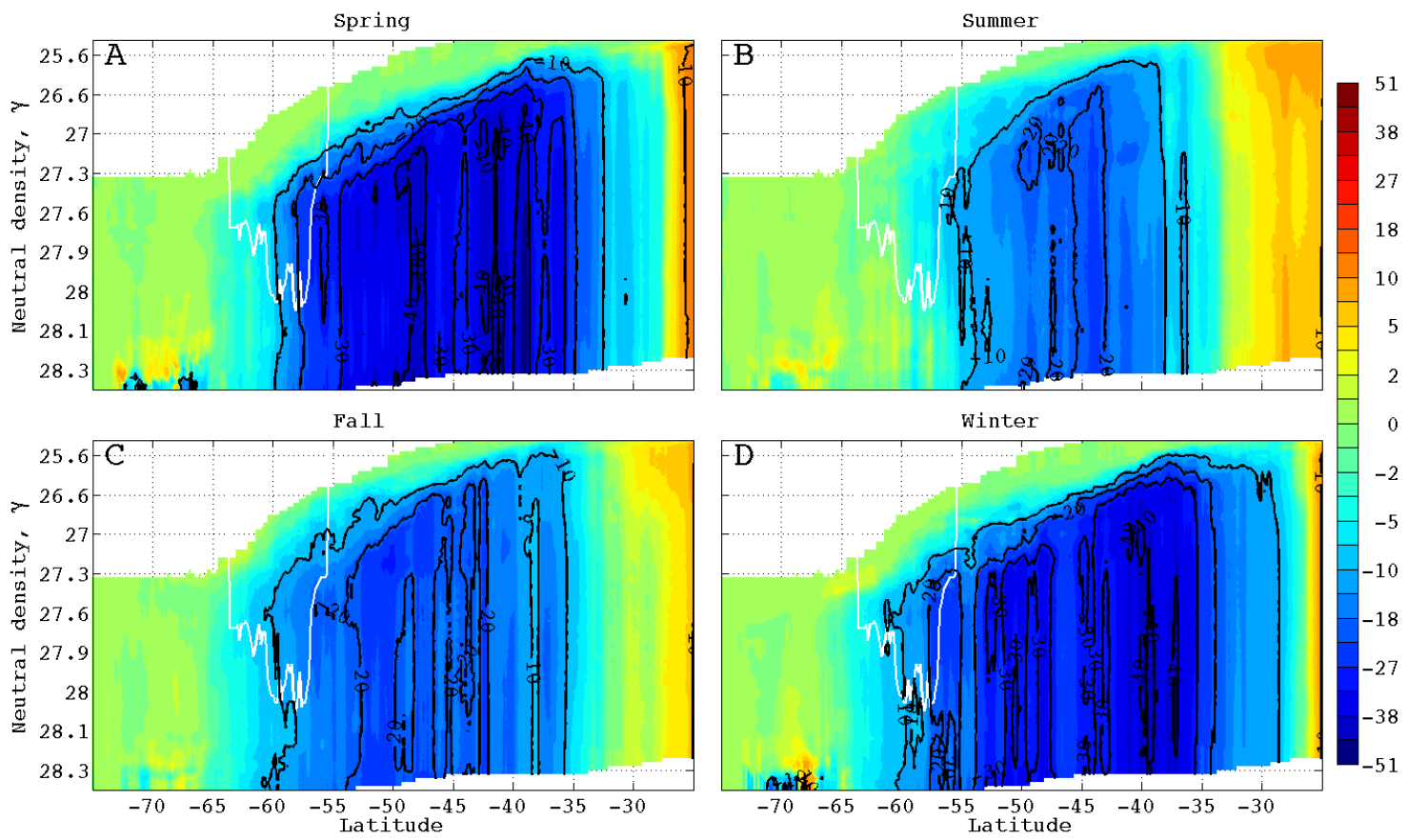

Figure 4-3: The ageostrophically balanced volume weighted mean flow in Sv. This streamfunction is constructed by adding the mechanical forcing (Figure 4-1) and the eddy potential vorticity fluxes (Figure 4-2). This figure is comparable to Figure (4-1).

mately $10 \mathrm{~Sv}$ in the summer, and up to approximately $30 \mathrm{~Sv}$ in the winter. The eddy PV flux exhibits little structure (divergence) in the greater density classes. Note this eddy PV flux is in disagreement to what one would expect from the theories of the nature of Johnson and Bryden (1989); the eddy PV flux does not oppose the Ekman transport. The reason for this result is explained in Section (4.3.8).

Combining the flow balanced by eddy PV fluxes with the flow balanced by direct mechanical forcing gives the lowest order ageostrophic residual circulation (Figure 4-3). In general, this circulation shows a cell (approximately $20 \mathrm{~Sv}$ in the summer and fall, and approximately $40 \mathrm{~Sv}$ in the winter and spring) that upwells in the Drake Passage latitudes $\left(\sim 60^{0} S\right)$, and downwells at the Subtropical Front $\left(\sim 35^{0} S\right)$. One may wish to think of this as an ageostrophic Deacon Cell.

There is a small contribution to the residual circulation streamfunction owing to the presence of the Gent-McWilliams-Redi (GM) eddy flux parameterization in the state estimate (see Section 3.2.4). Between $65^{0} \mathrm{~S}$ and $45^{0} \mathrm{~S}$ this GM streamfunc- 

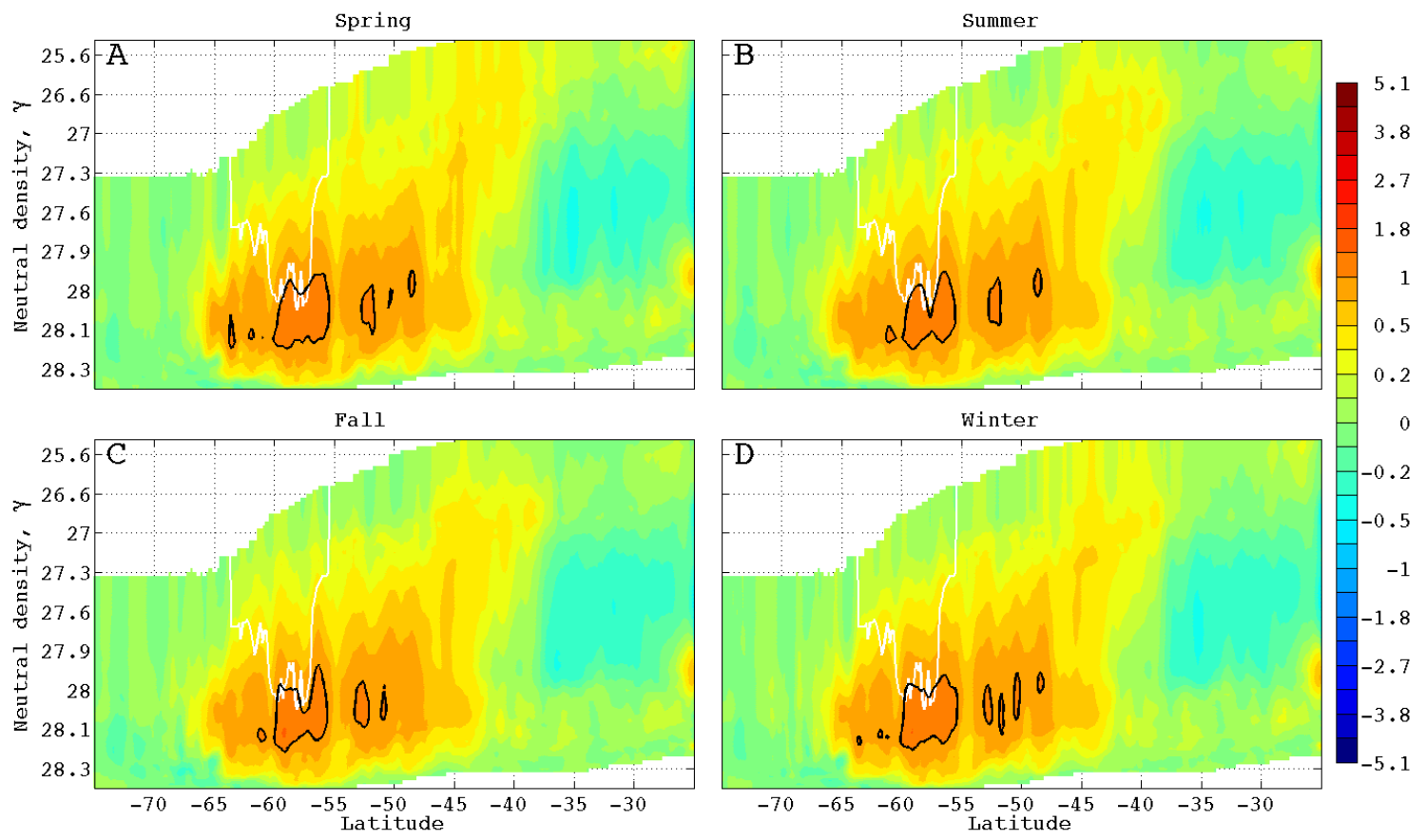

Figure 4-4: The parameterized transport [Sv] from the Gent-McWilliams-Redi (GM) eddy flux parameterization in the state estimate. This figure is comparable to Figure (4-1), exceptions being that the color-axis has been decreased by an order of magnitude and the contour interval is $1 \mathrm{~Sv}$.

tion acts in concert with the abyssal cell of the geostrophic streamfunction (see next section), transporting $\sim 1 \mathrm{~Sv}$ of deep waters poleward, and $\sim 1 \mathrm{~Sv}$ of bottom waters equatorward (Figure 4-4). As this parameterization represents mesoscale eddy effects, it should be considered comparable to the resolved eddy PV flux (Figure 4-2) and included in the ageostrophic streamfunction. Because its transport is higher order, however, it has been omitted from all the plots in this section except Figure (4-7).

\subsubsection{The Geostropohic Residual Circulation}

The general pattern of the geostrophic streamfunction is in opposition to the ageostrophic streamfunction, with an approximately $20 \mathrm{~Sv}$ poleward near-surface flow that downwells around $60^{\circ} \mathrm{S}$ and upwells around $35^{\circ} \mathrm{S}$ (Figure 4-5). The streamfunction diminishes at intermediate densities where a $\sim 10$ Sv equatorward flow of intermediate water is returned geostrophically. In deeper density classes the streamfunction in- 

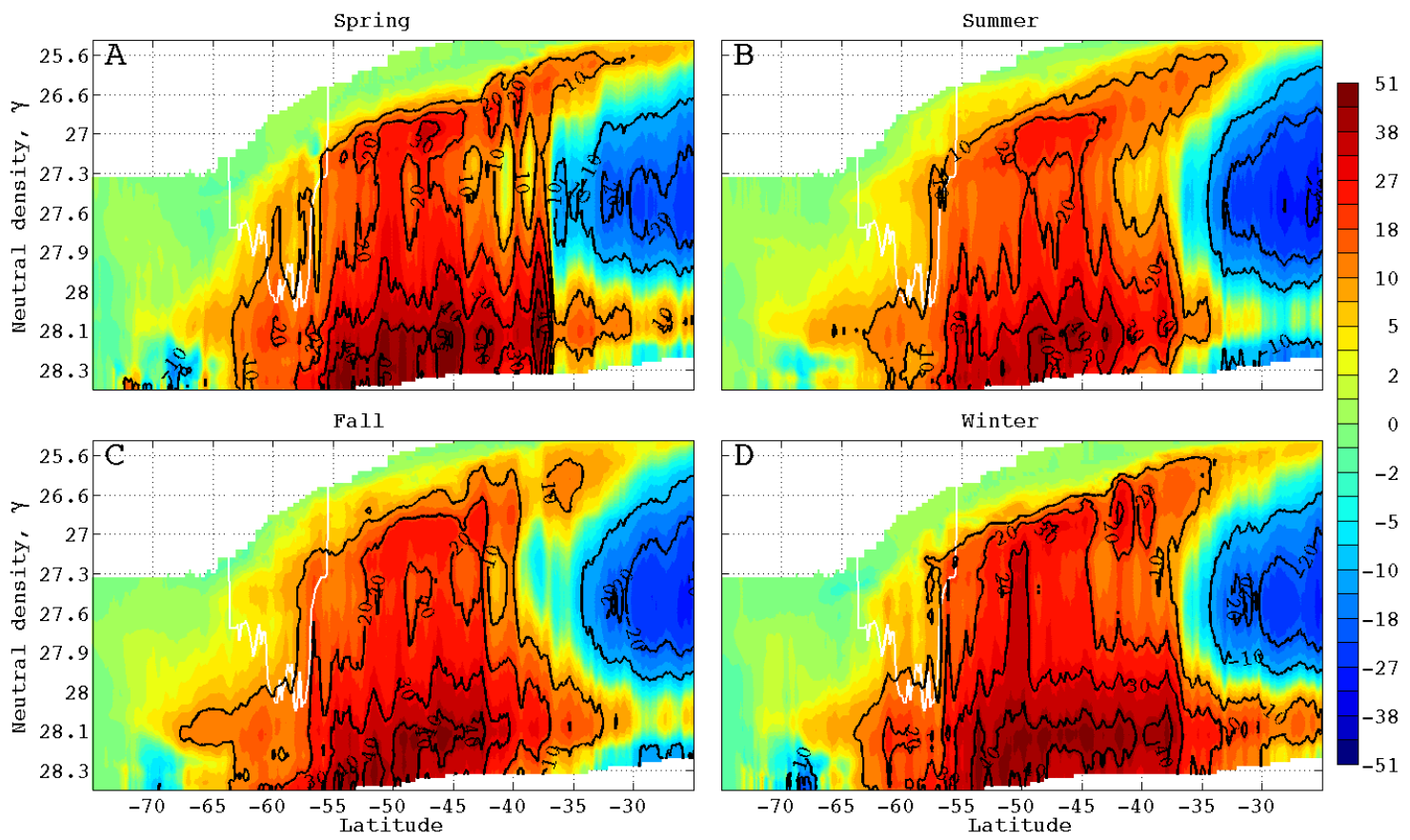

Figure 4-5: The full pressure balanced transport, $\left(\rho_{0} \bar{P}^{*}\right)^{-1} \overline{p_{x}}$, in Sv. This figure is comparable to Figure (4-1).
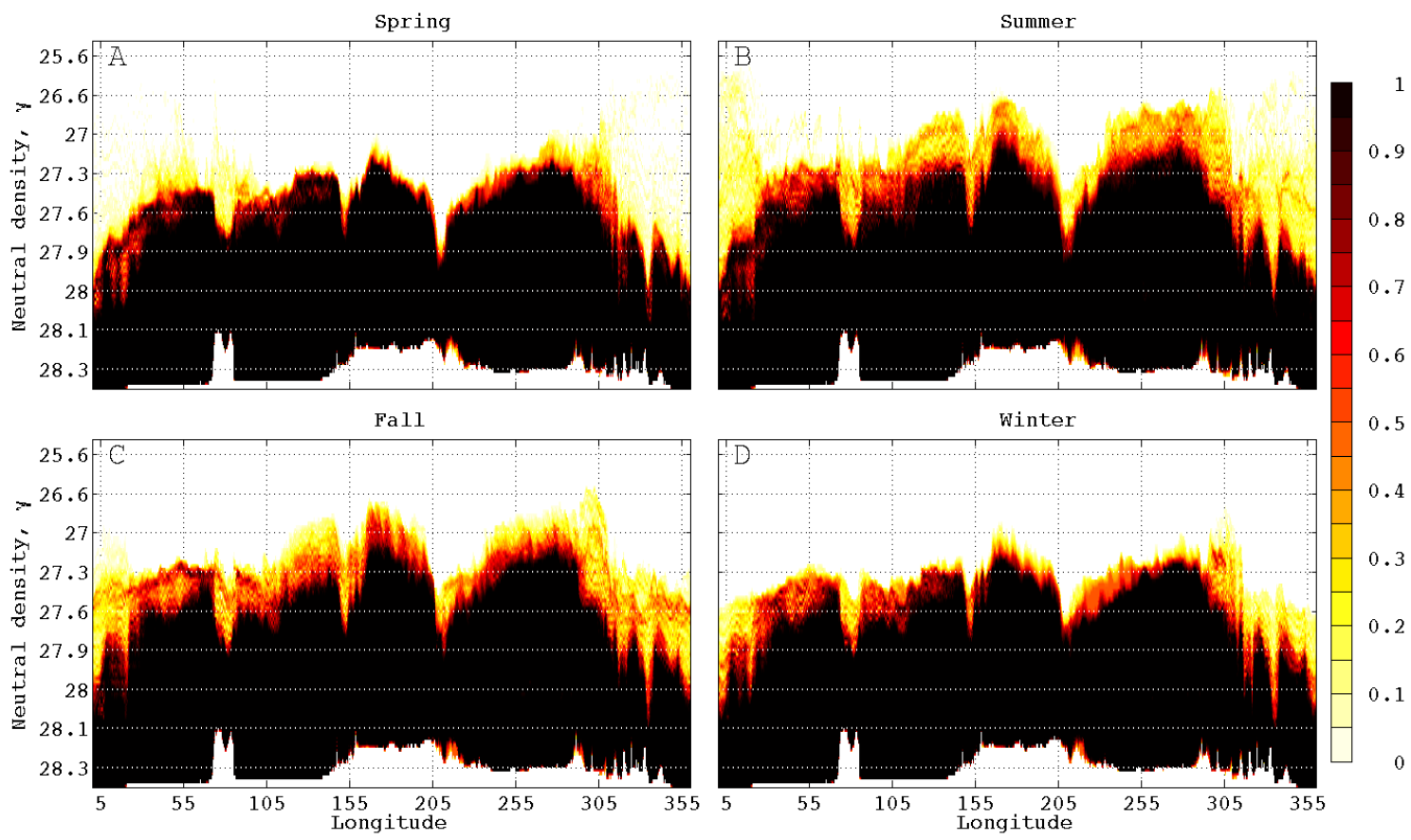

Figure 4-6: The probability that an isopycnal layer exists in the Southern Ocean state estimate, $\mathcal{A}$, for the $59.6^{0} S$ meridional section. A value of one means that for the given location and season, the isopycnal layer is present at all times. 
creases in strength with a $\sim 20 \mathrm{~Sv}$ poleward flow of circumpolar deep waters, and then diminishes again as $\sim 10 \mathrm{~Sv}$ of bottom waters flow geostrophically out of the Southern Ocean. The geostrophic flow is, of course, quite different equatorward of $35^{0} \mathrm{~S}$ in the sub-tropical gyres.

There is a noticeable reduction of the geostrophic transport to under $10 \mathrm{~Sv}$ in the unblocked latitudes. In these latitudes interior pressure gradients vanish, and pressure forcing can only be accomplished via isopycnal outcrops. The circulation associated with these surface outcrops is weaker than that associated with topographically induced pressure gradients. Nevertheless, the circulation, and thus zonal outcropping, is significant.

The probability of an isopycnal existing at a given time in a given season,

$$
\mathcal{A}\left(x, y, \gamma_{i}\right) \equiv \iint_{\gamma_{b}}^{\gamma_{s}} \delta\left(\gamma_{i}-\gamma\right) d \gamma d t\left(\int d t\right)^{-1}
$$

has been calculated for the Southern Ocean state estimate. A value of $\mathcal{A}=1$ means that for a given season and location, water of density $\gamma_{i}$ is present at all times. Analysis of $\mathcal{A}$ shows that almost all isopycnals outcrop zonally (into the bottom or surface) at some point and time. In fact, for the state estimate's $1 / 6^{0}$ resolution grid and the 90 defined $\gamma$ levels, only in eight places are there continuous integration paths over an entire season. There are three locations where this happens in summer, one in fall, none in winter, and four in spring. They all occur at approximately $\gamma=28.14 \mathrm{kgm}^{-3}$ (the approximate density of Lower Circumpolar Deep Water) and latitude $59.6^{0} \mathrm{~S}$. Plotting $\mathcal{A}$ at this latitude (Figure 4-6) shows that the surface zone "topography" spans many more neutral density classes than does the bottom topography. It is primarily the combination of latitude circles crossing the polar front into the eastern Weddell Sea and causing surface outcrops, and the Kerguelen Plateau at $70^{\circ} \mathrm{E}$ causing bottom outcrops, that breaks the circumpolar pathway. The Campbell Plateau at $155^{\circ} \mathrm{E}$ and the Pacific-Antarctic Ridge to its east are also notable constriction points. 

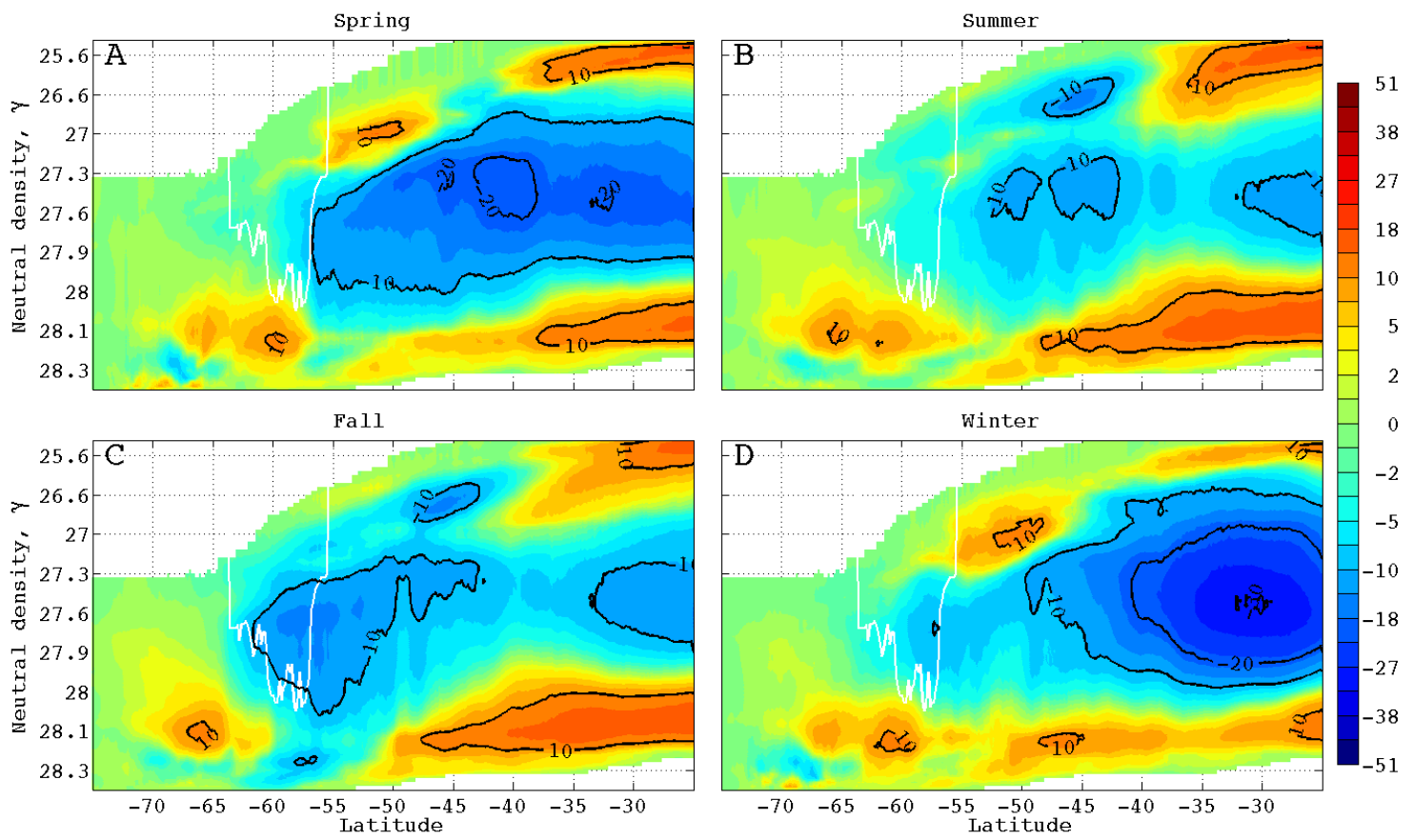

Figure 4-7: The volume weighted meridional circulation in the Southern Ocean state estimate in $\mathrm{Sv}$ (term $\bar{h} \bar{v}^{*}$ of Equation 4.20). This figure is comparable to Figure $(4-1)$.

\subsubsection{The Total Residual Circulation}

In the Southern Ocean, eddy potential vorticity (PV) flux forcing and wind forcing are largely balanced by pressure forcing. Thus the Southern Ocean ageostrophic and geostrophic overturning streamfunctions are in opposition. The residual of these two circulations, Figures (4-3) and (4-5), gives the volume weighted transport streamfunction (Figure 4-7; this circulation has also been discussed as a yearly average in Section 3.2.4). (Note that though it is rather insignificant, the parameterized transport from the Gent-McWilliams-Redi eddy flux parameterization, Figure (4-4), has been included in Figure (4-7).) For comparison the ageostrophic and geostrophic overturning streamfunctions are combined in Figure (4-8). There are some significant differences between Figures (4-7) and (4-8), for example a diffusive flux driven transport is significant at the open northern boundary in the spring and winter, and this is omitted from Figure (4-8). Many of the differences, however, are likely due to numerical issues in the calculation of the forcing terms. The terms must be integrated 

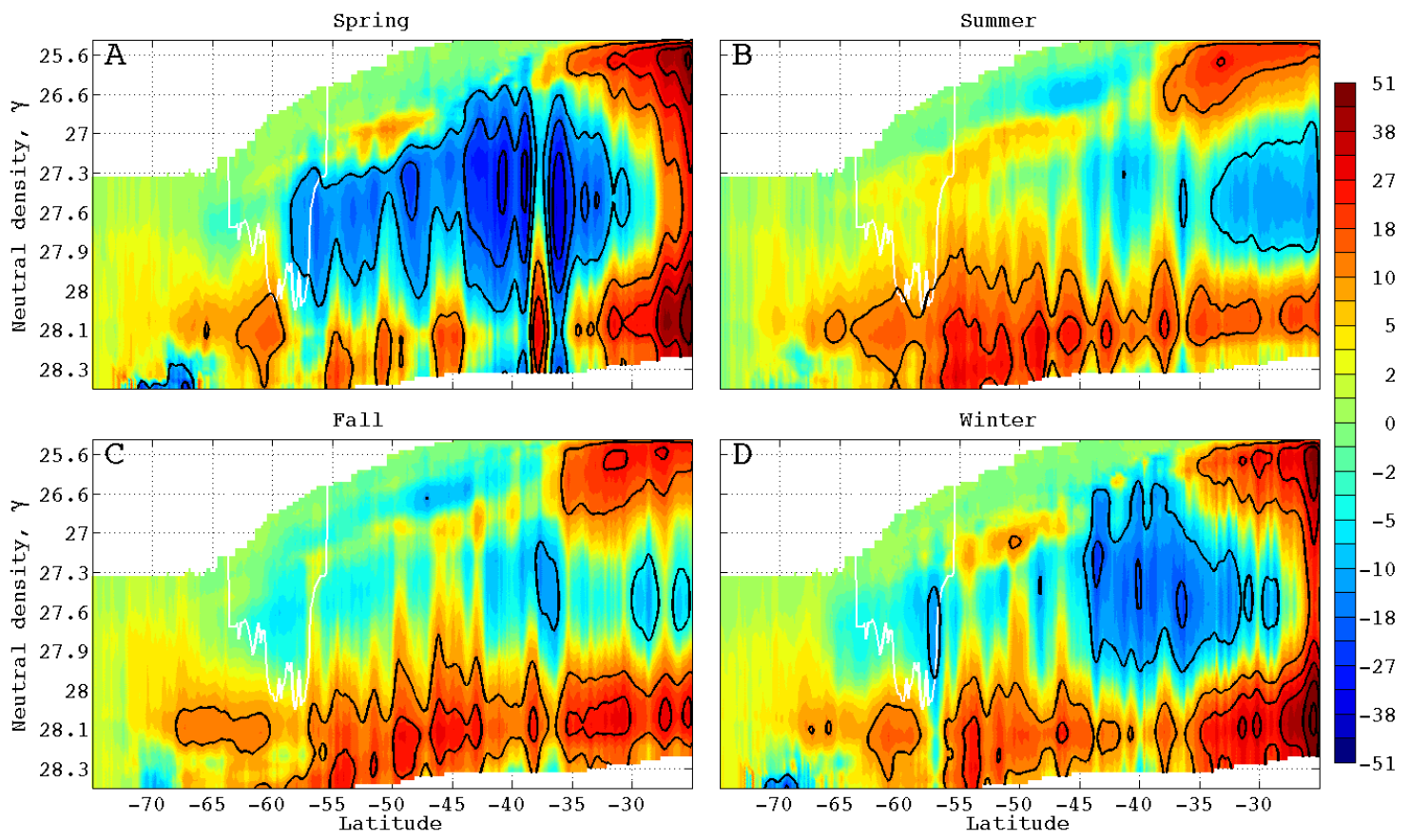

Figure 4-8: A combination of the ageostrophic overturning streamfunction (driven by mechanical forcing and eddy PV fluxes) and the geostrophic overturning streamfunction (i.e. Figure (4-3) plus Figure (4-5)). This residual streamfunction should be compared to that calculated directly in Figure (4-7). These plots are noisy, and there are some issues at the prescribed northern boundary, yet it is apparent that the general structure of the residual streamfunction is captured by combining the mechanical forcing, eddy PV flux, and pressure gradient terms. (This figure is comparable to Figure (4-1).)

in time and also in $x, z$, and $\gamma$ space; thus small numerical biases may become large signals over these significant integrations. The terms are also divided by the mean potential vorticity, and this may cause outliers to appear in the integrals in regions where the mean PV becomes exceptionally small. No cropping or smoothing was carried out on the terms calculated and plotted in this section. Despite these issues, it is apparent that one can reproduce the general features of the residual overturning circulation through consideration of mechanical forcing, eddy PV fluxes, and pressure gradients.

The geostrophic streamfunction overcomes the ageostrophic streamfunction in the lightest density classes. These lightest density classes dominate the buoyancy flux (shown below), and thus it is the poleward geostrophic flow that is responsible for 
closing the buoyancy budget. This poleward geostrophic flow is associated with the poleward excursion of the ACC as it crosses the South Indian and Pacific Oceans. The surface waters of the ACC are more buoyant when they are moving southward across the Pacific Ocean than when they are moving northward as the Falkland (or Malvinas) Current. At intermediate densities a combination of the strengthening of the equatorward ageostrophic transport and a reversal of the geostrophic transport allows intermediate waters to flow equatorward and ventilate the sub-tropical oceans. In denser water classes a poleward geostrophic transport brings circumpolar deep waters into the Southern Ocean. An abyssal equatorward geostrophic flow expels bottom waters from the Southern Ocean and closes the residual circulation. The characteristic multiple cell structure of the Southern Ocean residual circulation is attributable to variations of the geostrophic streamfunction over density classes.

There are significant differences between the summer to fall residual circulation, and the winter to spring circulation (Figure 4-7). The primary difference results from an upward shift in density space of the ageostrophic streamfunction. In the summer and fall the equatorward flow driven by the winds and eddy PV fluxes acts, on average, on the lightest density classes. In these density classes the ageostrophic forcing dominates over the pressure gradients driving all but the most buoyant surface waters equatorward. In the winter and spring the zonally integrated ageostrophic forcing acts primarily on less buoyant density classes, and thus the more buoyant waters are driven polewards by the dominant pressure gradient.

Ageostrophic forcing, both from eddy potential vorticity fluxes and atmospheric wind stresses, drives a significant upwelling in the unblocked latitudes (Figure 43). The geostrophic circulation is relatively weak at these latitudes and thus a net upwelling occurs in the residual circulation. If the wind stress divergence increased, it is likely that more deep waters would be brought to the surface. This would result in more isopycnal outcrops, and thus a stronger geostrophic circulation could be sustained to counter the upwelling. Perhaps this balance is occurring to some degree since in the spring, when the zonal wind stress divergence is the greatest, the number of outcrops is greatest (see Figure 4-6), and the geostrophic streamfunction in the 
unblocked latitudes is strongest.
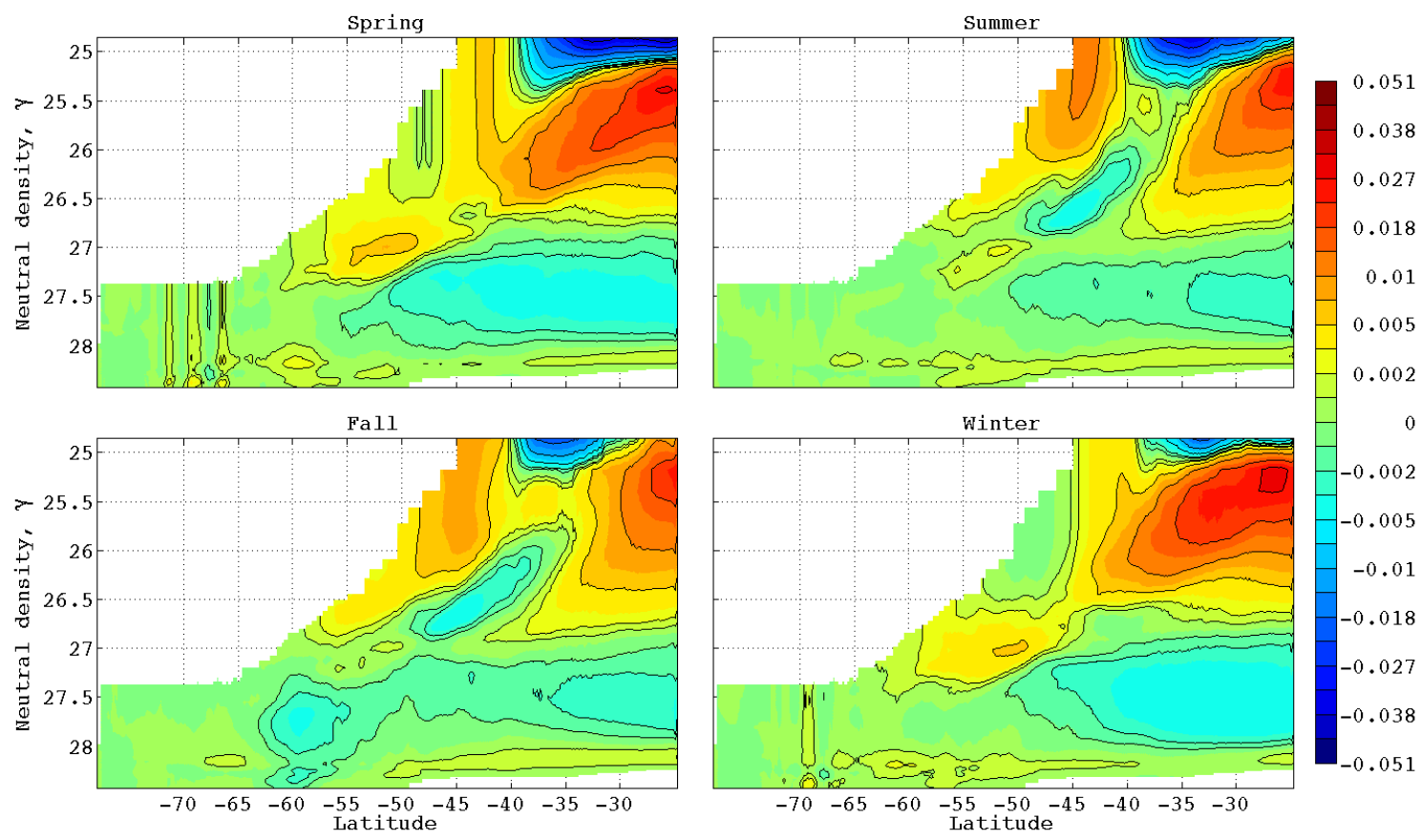

Figure 4-9: The buoyancy transported by the residual circulation in $m s^{-2} S v$. A positive buoyancy streamfunction implies counter-clockwise transport. For latitudes in which all density classes are plotted (i.e. south of $\sim 40^{0} \mathrm{~S}$ ) there is a net poleward transport of buoyancy that is established by the lightest density classes. Note the $\mathrm{y}$-axis is linear, demonstrating the significant density span in near surface waters.

\subsubsection{Buoyancy Fluxes}

It is the residual circulation, $\Psi^{*}$ (Figure $4-7$ ), that advects water properties. The transport of buoyancy, $b \equiv g \gamma_{0}^{-1}\left(\gamma_{0}-\gamma\right)$, is thus given by $\int_{\gamma_{b}}^{\gamma} \Psi^{*} d b$ (Figure 4-9). For latitidues in which all density classes are plotted (i.e. south of $\sim 40^{\circ} \mathrm{S}$ ) a net poleward transport of buoyancy is apparent. This transport, which extends to at least $60^{\circ} \mathrm{S}$, is carried primarily in the lightest density classes. One should evaluate this buoyancy transport only qualitatively, as its magnitude is proportional to the unknown constant $\gamma_{0}$ (see the discussion of $\gamma_{0}$ at the beginning of this section). Heat and salt fluxes are both considered quantitatively, however, in Section (3.2.2). That analysis showed that in the Southern Ocean, heat and salt are both transported poleward. A heat (salt) transport is associated with a positive (negative) buoyancy transport. The 
poleward buoyancy transport therefore implies that, for this region (north of $60^{0} \mathrm{~S}$ ), temperature fluxes dominate the buoyancy budget.
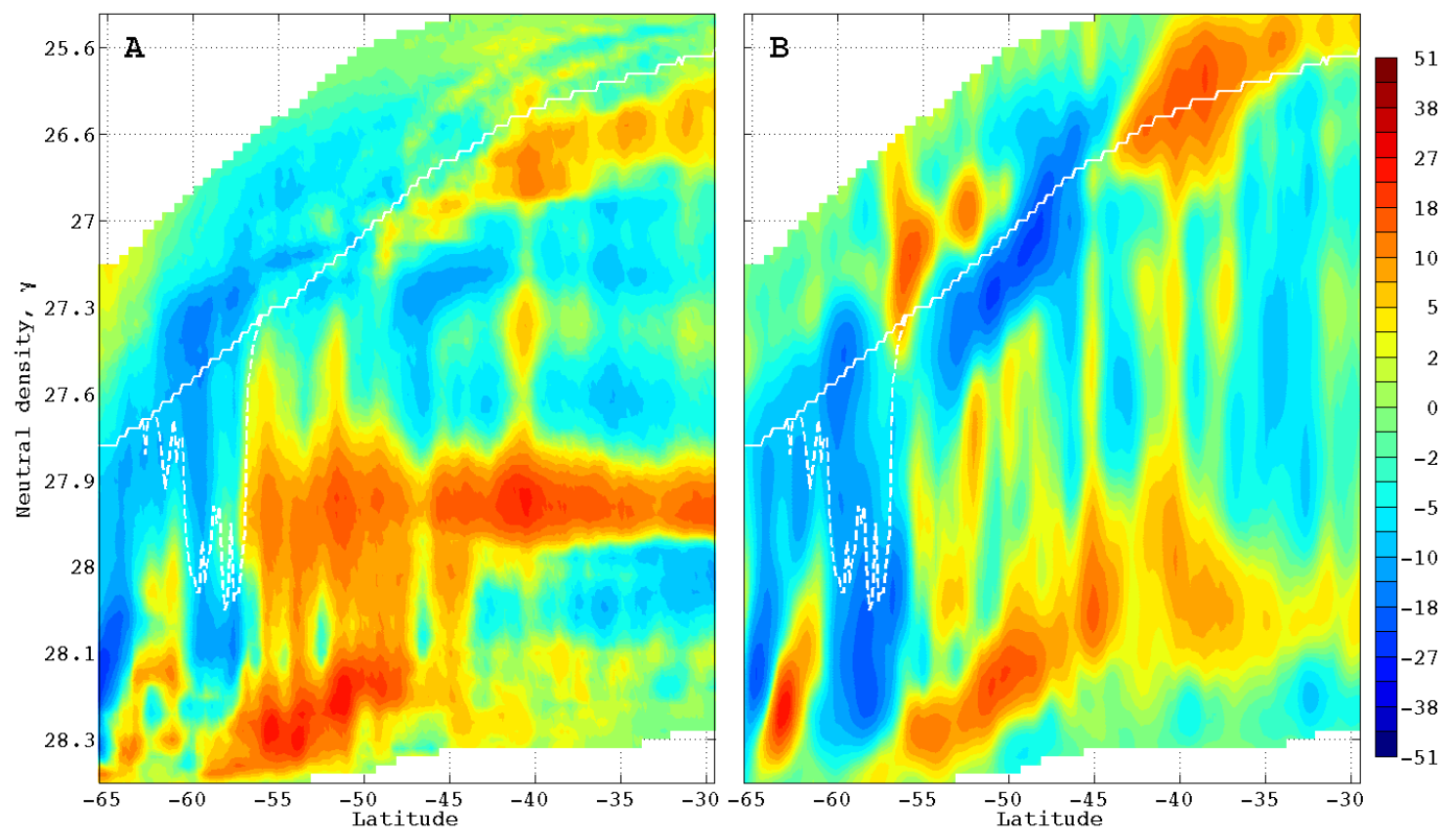

Figure 4-10: The mean area tendency, $\int_{z\left(\gamma_{b}\right)}^{z\left(\gamma_{n}\right)} A_{t} d \gamma$, (Panel A) and mass flux, $\int_{\gamma_{b}}^{\gamma_{n}} \Psi_{y}^{*} d \gamma$, (Panel B) for the state estimate. An area tendency signifies a volume change within an isopycnal layer, and this area change appears as an apparent buoyancy source in the residual circulation. Comparison of Panel A and B shows that this is occurring in several regions in the state estimate. Differences between the two panels are a result of buoyancy forcing and diffusive fluxes. The fields have been smoothed for better presentation. The color-axis and ordinate are non-linear.

\subsubsection{Model Drift}

The conservation budget for a zonal and vertical cross-section of an isopycnal layer bounded by $\gamma_{2}<\gamma<\gamma_{1}$ is

$$
A_{t}=-(\partial / \partial y) \oint \int_{z\left(\gamma_{1}\right)}^{z\left(\gamma_{2}\right)} v d z d x+D_{\gamma}+\mathcal{B}
$$

where $A=\oint \int_{z\left(\gamma_{1}\right)}^{z\left(\gamma_{2}\right)} d z d x$ is the area of the layer, $D=\oint \int_{z\left(\gamma_{1}\right)}^{z\left(\gamma_{2}\right)}\left(k \gamma_{z}\right)_{z} d z d x=\left.\oint k \gamma_{z}\right|_{z\left(\gamma_{1}\right)} ^{z\left(\gamma_{2}\right)} d x$ is the diffusion in the layer, and $\mathcal{B}$ is the air-sea area flux. The term $\oint \int_{z\left(\gamma_{1}\right)}^{z\left(\gamma_{2}\right)} v d z d x$ is the advective flux in the layer cross-sections. 
Setting $\gamma_{1}=\gamma_{b}$, where $\gamma_{b}$ is at (or below) the ocean floor one finds

$$
\Psi_{y}^{*}=A_{t}-D_{\gamma}-\mathcal{B}
$$

Integrating this in $\gamma$, from $\gamma_{b}$ to an interior level, $\gamma_{n}$,

$$
\int_{\gamma_{b}}^{\gamma_{n}} \Psi_{y}^{*} d \gamma=\int_{\gamma_{b}}^{\gamma_{n}} A_{t} d \gamma-\left.D\right|_{\gamma_{b}} ^{\gamma_{n}}
$$

Air-sea fluxes are zero in the interior, $\mathcal{B}=0$, and there is no geothermal heating in the state estimate, making the diffusive flux negligible at the ocean floor. Assuming a stable stratification $\left.D\right|_{\gamma_{b}} ^{\gamma_{n}}=\oint k \gamma_{z} d x>0$; diffusion mixes $\gamma$ upwards (buoyancy downwards). In the absence of an area increase one would therefore expect that the mass flux integrated from the bottom to $\gamma_{n}$ to be decreasing with latitude (i.e. $\left.\int_{\gamma_{b}}^{\gamma_{n}} \Psi_{y}^{*} d \gamma<0\right)$. In the densest water classes, where there are no air sea fluxes, the mass flux is, for the most part, decreasing with latitude (see Figure 4-9: at constant $\gamma$, the values should be decreasing equatorward). There are a few interior regions, however, where the mass flux increases with latitude. These occur predominantly in the density classes greater than $\gamma=27.8 \mathrm{kgm}^{-3}$, northward of $65^{0} \mathrm{~S}$, and most significantly in the fall. It has been found that though buoyancy forcing and diffusive fluxes are significant in select regions, for this region of concern the abyssal mass flux is largely balanced by the area tendency, $\int_{\gamma_{b}}^{\gamma_{n}} A_{t} d \gamma$. This area tendency change accounts for changes in the residual streamfunction of order $10 \mathrm{~Sv}$, and in this abyssal region it accounts for changes of up to $18 \mathrm{~Sv}$ (Figure 4-10). What appears to be an abyssal buoyancy source in the residual circulation is actually a change in the volume within an isopycnal layer. In the absence of this model drift the residual circulation (Figure 4-7) would look qualitatively different.

The isopycnal layer volume change is attributed to model drift. It is likely that much, if not all of the drift is due to model inconsistencies (e.g. between the surface forcing and the initial or the northern boundary conditions), as opposed to a trend in the Southern Ocean observations. The drift is acting to change the stratification of the solution, there is no significant acceleration of the flow. A thickening and 
thinning the isopycnal layers is occurring, and thus the location of the $\gamma$ levels in $z$-space is changing. As a result, the "window" through which the zonal momentum balance is being viewed is moving in time. Because the overturning circulation has significant vertical structure, this movement is causing apparent artifacts and changes in the circulation. This is not a change in the force balance, however, it is simply a change in how the dynamics are being viewed. Comparing the 2005 and 2006 residual streamfunctions (Figure 4-11) shows an apparent drift in the circulation is largely geostrophically balanced. This suggests that while model drift may cause spurious effects in the averaging, and thus plotting, of the momentum balance, the lowest order balance presented in this thesis is a robust result.
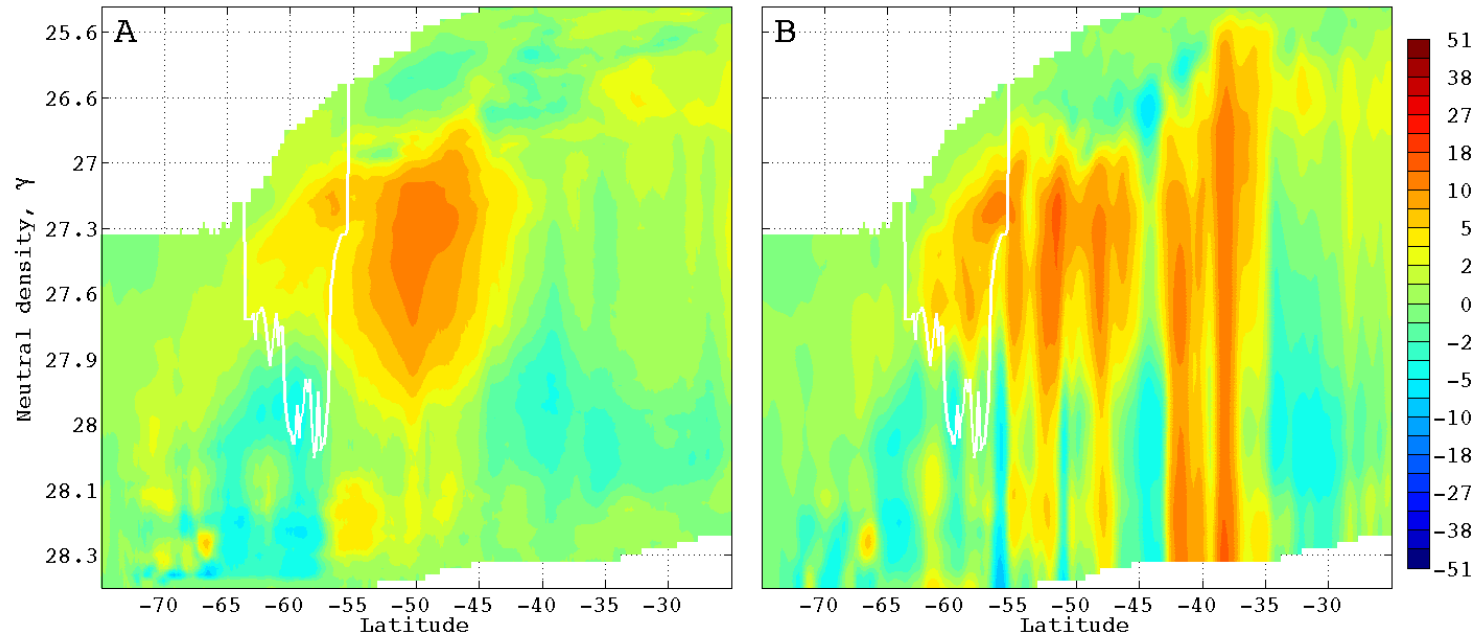

Figure 4-11: The yearly change (average value in 2006 minus the average value in 2005 ) of the residual streamfunction, $\Psi^{*}$, (Panel A), and of the geostrophic streamfunction (Panel B). The residual overturning of the intermediate cell is reduced by about $7 \mathrm{~Sv}$, and the deep cell has changed by several Sverdrups. These perceived changes are largely spurious; when $\gamma$ levels move vertically the streamfunction strength will change due to shear in the velocity field. The change in the streamfunction are more due to changes in the stratification, however, as opposed to an actual transport increase. The changes (drift) in $\Psi^{*}$ appear to be largely in geostrophic balanced. 

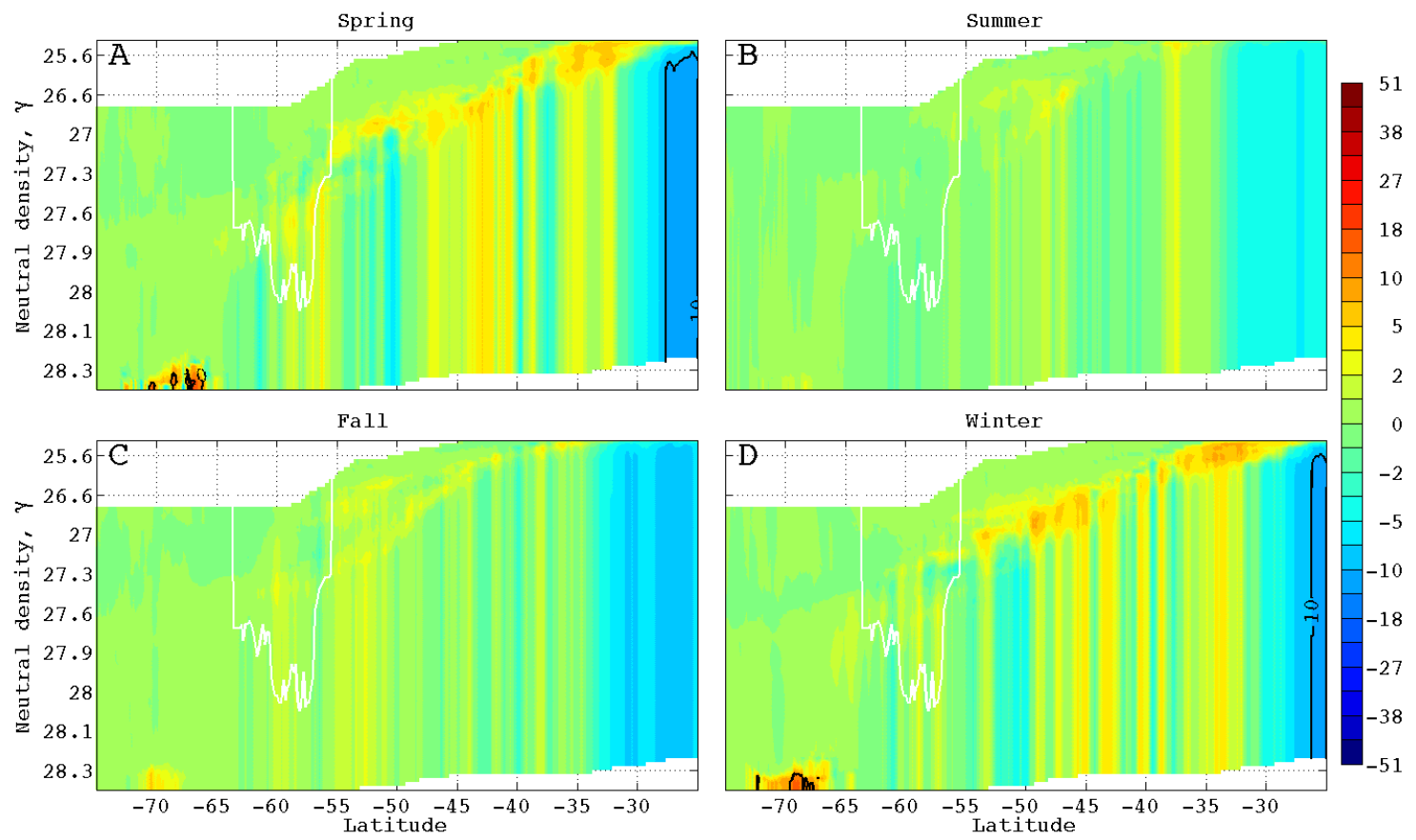

Figure 4-12: The component of the pressure balanced transport associated with surface buoyancy fluxes in $\mathrm{Sv}\left(\operatorname{term}\left(\rho_{0} \bar{P}^{*}\right)^{-1} \overline{f v_{g} \rho \delta\left(\gamma-\gamma_{s}\right)}\right.$ of Equation 4.20). This figure is comparable to Figure (4-1).

\subsubsection{Surface Buoyancy Fluxes}

Pressure forces acting on outcrops, which balance a surface geostrophic circulation, can be represented in terms of a surface geostrophic mass flux (Equation 4.18). This circulation is characterized by a poleward flow in the lightest density classes at almost every latitude (Figure 4-12). A recirculation of this surface circulation is apparent, as denser surface waters move equatorward. Meridional oscillations (meanders) of water masses are primarily responsible for this flow. When an eddy or a jet meanders equatorward and gains buoyancy, then meanders southward and loses buoyancy, an overturning in density space results (assuming the buoyancy gain and loss occur with different displacement dependencies). Though the circulation balanced by this induced pressure forcing is relatively small, with a magnitude of $\sim 2 \mathrm{~Sv}$ in the summer and fall, and a magnitude of $\sim 7 \mathrm{~Sv}$ in the winter and spring, it occurs in the lightest density classes, and is thus quite significant to the buoyancy budget (Figure 4-9). This result is consistent with the fact that in the ACC latitudes the buoyancy flux is 
governed by transient dynamics (Section 3.2.2).
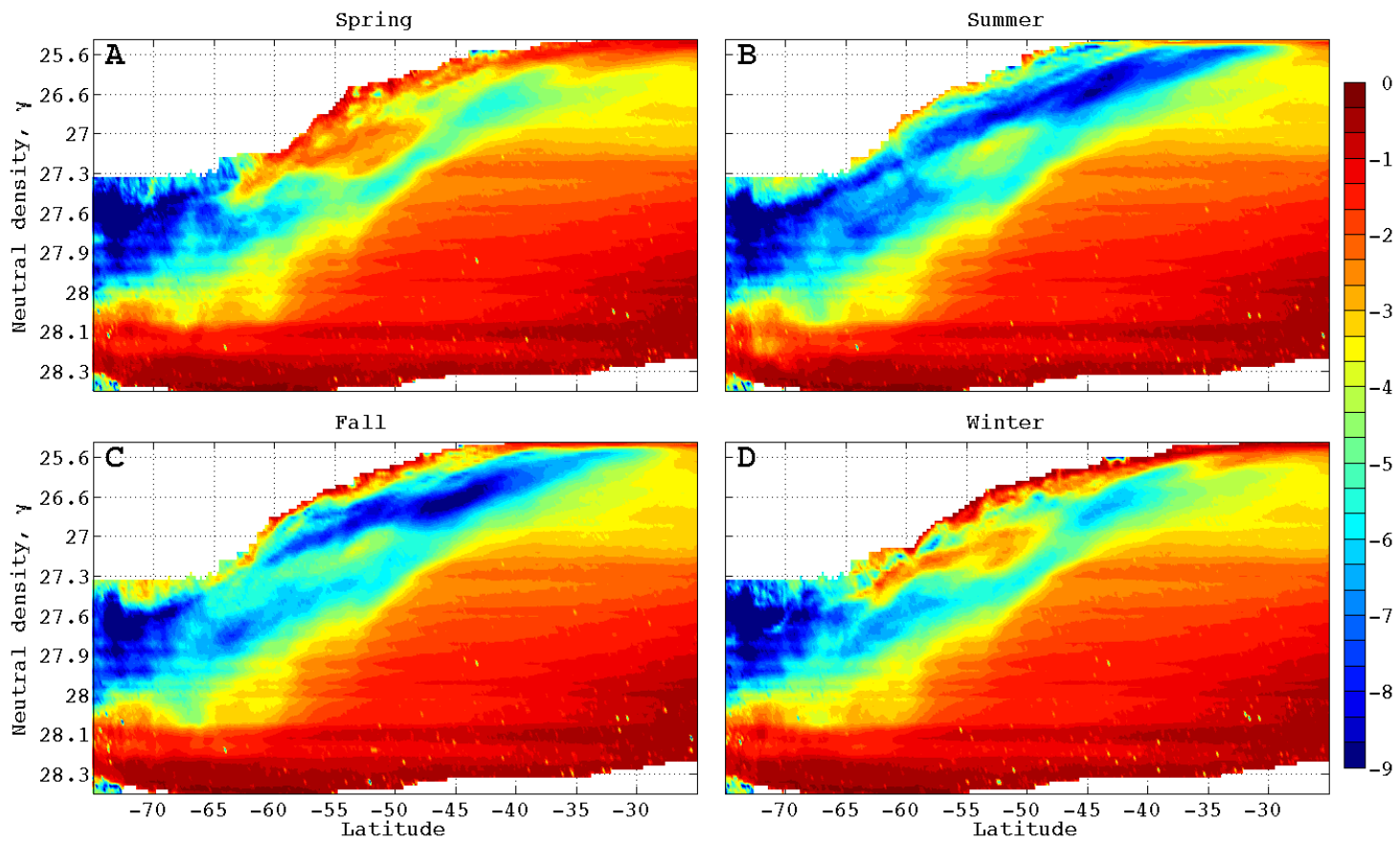

Figure 4-13: The mean potential vorticity, $f / h$, in $(m s)^{-1}$ where $h$ is an isopycnal layer thickness, $h \approx \rho_{0} \rho_{z}{ }^{-1}$, for the state estimate. Note the ordinate is stretched in $\gamma$-space.

\subsubsection{The Eddy Potential Vorticity Flux}

Eddy potential vorticity (PV) fluxes balance a significant ageostrophic residual circulation in Southern Ocean (Figure 4-2). Modern climate models cannot afford to resolve eddy effects, and rely on eddy parameterizations. The parameterizations of these mesoscale dynamics depends on the assumption that eddy fluxes are related to the along-isopycnal PV gradient, e.g. $\hat{\mathrm{P}}^{*}=-\left.k \bar{P}_{y}^{*}\right|_{\gamma}$. Eddy processes in the transformed Eulerian mean models of the nature of Marshall and Radko (2003) also employ these conventional down-gradient parameterizations (Olbers et al., 2004). Previous work has shown, however, that eddy potential vorticity fluxes are not simply related to the mean PV field (Wilson and Williams, 2004).

In every season a potential vorticity minimum occurs at a density class just below the lightest waters (Figure 4-13). This minimum is associated with the thermocline, 

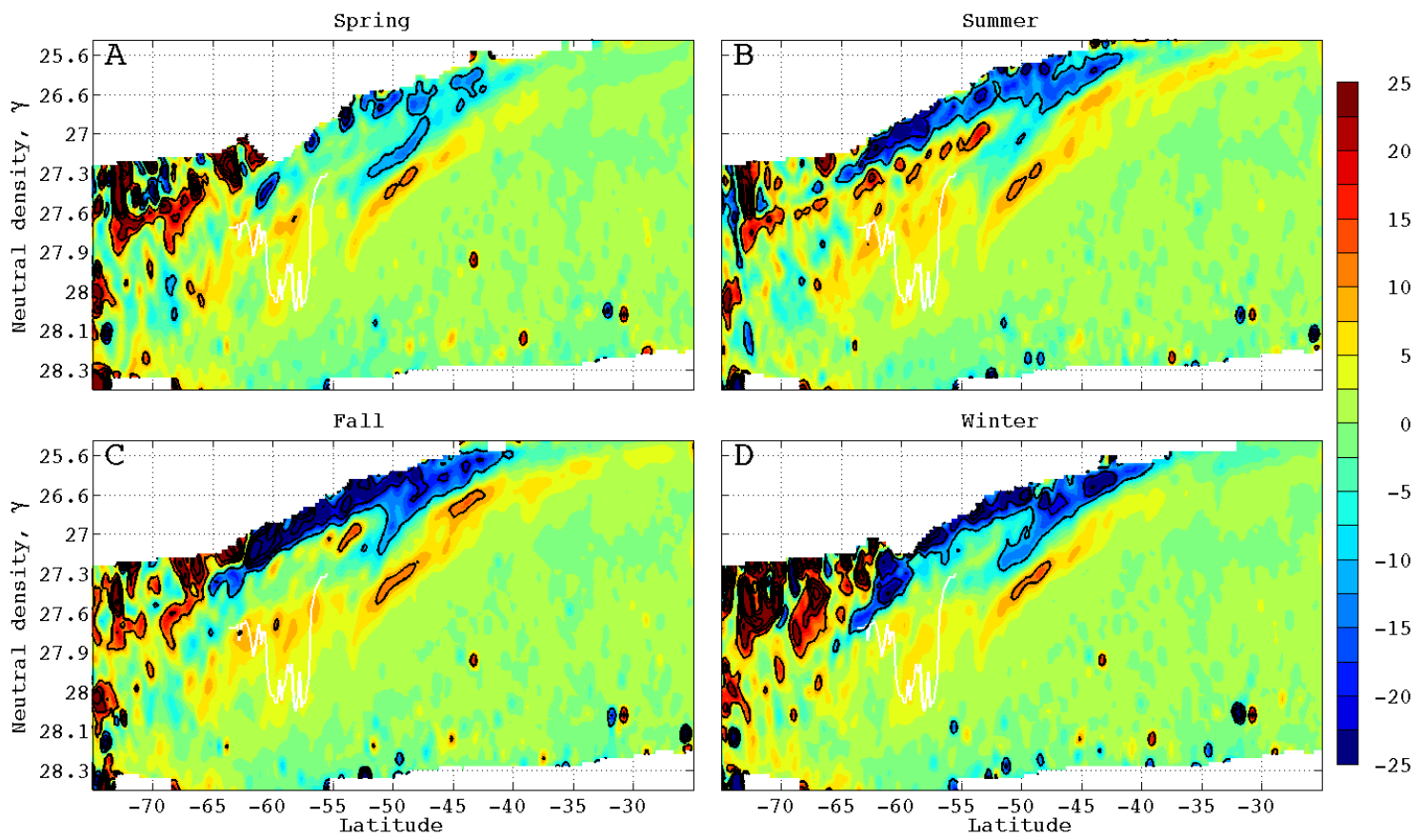

Figure 4-14: The zonally integrated potential vorticity gradient, $(f / h)_{y}$, in $\left.10^{-6}\right) s^{-1} m^{-2}$. The strongest gradients occur above the thermocline. Note there is a reversal in the PV gradient such that the necessary condition for baroclinic instability is satisfied (Pedlosky, 1987). The gradient has been smoothed for better presentation, and the ordinate is stretched in $\gamma$-space.

and is therefore most significant in summer and fall when the thermocline is strongest. Eddies tend to flux PV into this minimum; they are, for the most part, down-gradient (Figures 4-14 and 4-15). It has been suggested that eddy activity in the Southern Ocean occurs largely due to baroclinic instability processes (Johnson and Bryden, 1989). Here it is found that eddies are most significant near the surface. The $\hat{v}$ in the $\overline{\hat{P} \hat{v}}$ term has contributions from deviations in both the mean Ekman component, and in the mean geostrophic component. This is easier to see by making the good assumption that $P \approx f h^{-1}$, which allows the residual flux to be written as $\overline{v h}=$ $\overline{v^{\prime} h^{\prime}}+\overline{v_{g}} \bar{h}+\overline{v_{E k}} \bar{h}$, where the first term on the right hand side is equal to the eddy PV flux (see Figure 4-2), and the last two terms on the right hand side are equivalent to the pressure gradient driven circulation (see Figure 4-5) and the mechanically forced circulation (see Figure 4-1) respectively. This decomposition makes it apparent that $v^{\prime}=v_{g}^{\prime}+v_{E k}^{\prime}$. In the interior, where mechanical forcing is negligible such that $v^{\prime} \approx v_{g}^{\prime}$, 

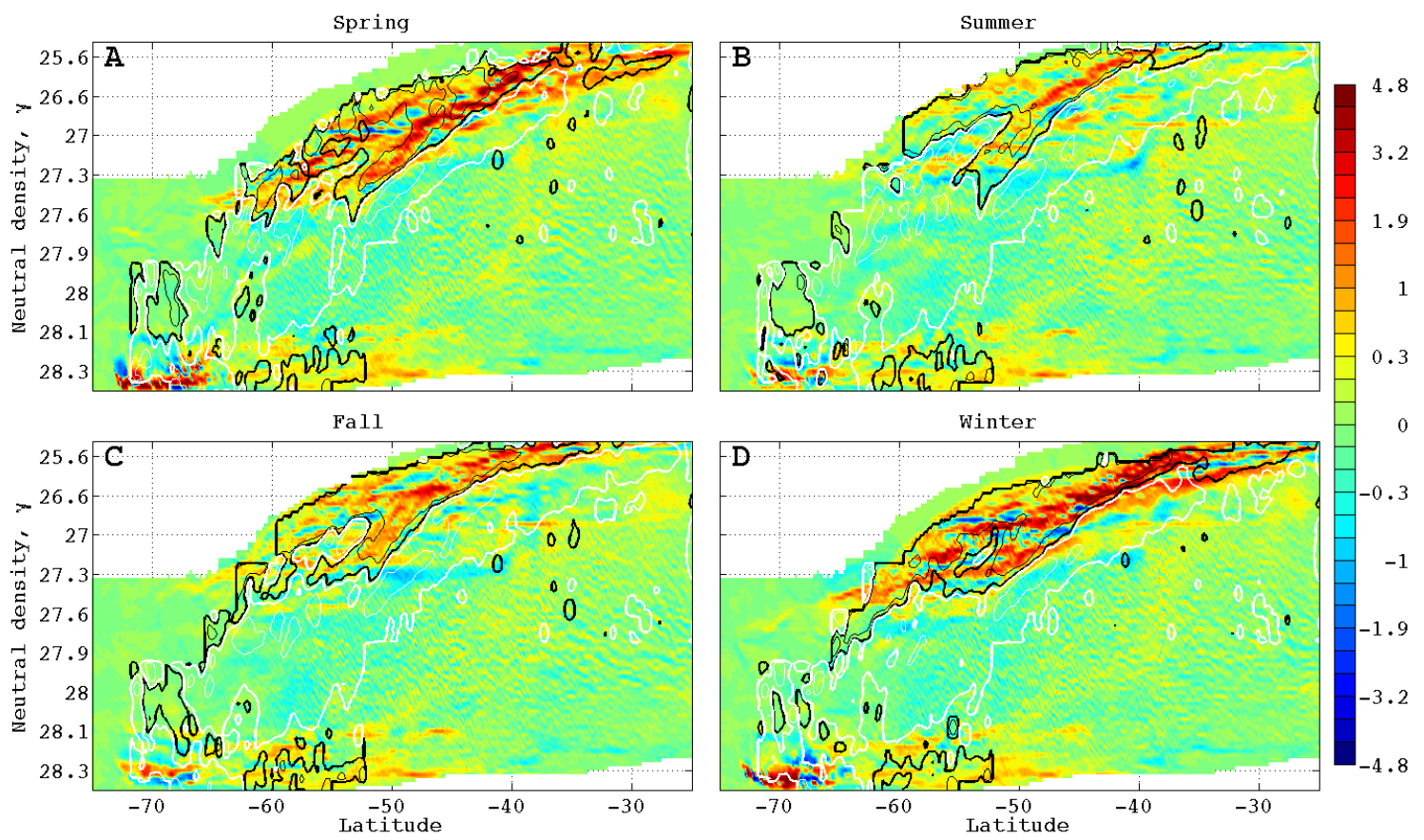

Figure 4-15: The eddy potential vorticity flux in $\mathrm{Sv}$ is plotted in color. Vertical integration has only been carried out in each layer as opposed to over the entire water column. This plot is therefore not a streamfunction, but the meridional layer transports associated with the streamfunction of Figure (4-2). Contours of the along isopycnal meridional gradient of potential vorticity are overlaid. The thick white (black) contours are of $(f / h)_{y}=10^{-6}\left(-10^{-6}\right) s^{-1} m^{-2}$, and thus enclose regions of positive (negative) potential vorticity gradient. The thin lines are contours of $7 \times 10^{-6}\left(-7 \times 10^{-6}\right) s^{-1} m^{-2}$, and thus represent regions of stronger gradients. A distinguishing feature of the plots is a large down-gradient potential vorticity flux in the lightest density classes (there is a large positive flux in the region of negative gradient).

baroclinic instability processes are a dominant source of eddy activity, and it makes sense to parameterize eddy activity as proportional to the mean buoyancy gradient. Near the surface, however, it is likely that wind variations drive the variability in the ocean circulation, and thus that $v_{E k}^{\prime}$ is larger than $v_{g}^{\prime}$. In this case, an appropriate eddy closure would likely reflect the magnitude of atmospheric variability, as opposed to the mean ocean state.

The eddy PV flux is not in agreement with the idealized theories of the nature of Marshall and Radko (2003). These theories propose that the circulation driven by down gradient PV fluxes should be poleward to cancel the Ekman transport. Figure 
(4-2), however, shows the opposite: the eddy PV flux driven circulation and the Ekman transport are both equatorward. A down gradient eddy PV flux would drive a poleward circulation for most of the ocean below the thermocline. The gradient above the thermocline, however, is strongly negative (Figure 4-14). This region is quite thin in $z$-space and has likely been overlooked. The state estimate analysis carried out here in density space has illuminated how important this relatively thin layer is in the dynamics. This layer dominates the buoyancy flux, and is also is the region where eddy PV fluxes are most significant.

Though the PV gradient immediately below the thermocline is weaker than the gradient above the thermocline, it is significant. It is not obvious why a stronger eddy PV flux driven circulation is not present in this region. The PV gradient does, however, become insignificant in the denser water classes. Therefore, as expected, PV fluxes induce little deep water transport. It is important to note, however, that the force balance being investigated is for an equilibrated ocean state. It is possible that eddies have already homogenized the interior PV. In this equilibrated state the interior eddy fluxes have vanished because there are no more PV gradients to stir (Kuo et al., 2005).

\subsection{Summary and Conclusions}

Southern Ocean literature often begins with a statement regarding this ocean's uniqueness due to the presence of the unblocked Drake Passage latitude band. It is commonly asserted that the net meridional geostrophic flow must vanish in these latitudes, and therefore freshwater, heat, and mass transport must be accomplished ageostrophically via mechanical forcing and eddies (Rintoul et al., 2001; Olbers et al., 2004)). Net pressure forcing does vanish in these latitudes when integrated zonally at constant depth. Fluid parcels, however, are believed not to move at constant depth, but instead to move primarily along locally reference potential density surfaces (or neutral density surfaces, (McIntosh and McDougall, 1996)). When averaged along these surfaces, the net zonal pressure gradients do not vanish from the zonal momentum balance. In fact, 
this forcing term occurs at lowest order. The densest water classes are in geostrophic balance. In the lighter density classes it has been found that eddies act in concert with mechanical forcing to generate a westward stress. Pressure forcing balances this stress, such that the difference between the two is a small residual. The residual stress is balanced by a Coriolis force generated via a net meridional transport.

The dominant terms in the zonal momentum balance in isopycnal space are given in Equation (4.20). The terms on the right hand side of this equation balance an overturning circulation, $\Psi^{*}$, composed of upper, intermediate, and lower cells (Figure 4-7). In the lightest of density classes pressure forcing, which results from interactions with both topography and surface outcrops, balances a poleward flow. While the volume transport of this upper flow is relatively small $(\sim 5 \mathrm{~Sv})$, it dominates the buoyancy flux (Figure 4-9). At intermediate density classes ageostrophic wind and eddy forces, and to some extent geostrophic pressure forces, drive an equatorward flow that closes the upper cell and forms the upper branch of the intermediate cell. This upper branch moves to lighter density classes in the summer and fall and largely cancels out the upper cell. Pressure forcing in the dense water classes drives fluid poleward to close the intermediate cell. A lower cell is also geostrophically balanced.

Eddy PV fluxes are higher order in equilibrated ocean interiors because interior PV gradients have already been largely homogenized along isopycnals. Eddy PV fluxes are most significant to the dynamics in the near surface region in and above the thermocline. In coarse resolution models turbulent eddy PV fluxes are represented using the Gent-McWilliams (GM) parameterization. This parameterization may break down when isopycnal gradients become extremely steep. Unfortunately, gradients often become steep near and above the thermocline. Thus the quality of the GM parameterization may be the weakest exactly where it is most necessary. Methods to deal with steep gradients in the parameterization of eddy fluxes may, therefore, significantly affect model solutions and should be implemented cautiously.

The results presented here have other implications for the GM eddy flux parameterization. Eddies may receive their kinetic energy from the release of potential energy via baroclinic instability processes. If this is the dominant source of eddy energy one 
would then expect the level of eddy activity to be set by the amount of oceanic available potential energy, and thus by the mean buoyancy gradient, $\bar{b}_{y}$. This work suggests, however, that much of the eddy PV flux arises from direct wind forcing. Parameterization coefficients meant to replicate the amount of eddy energy should therefore not be set proportional to $\bar{b}_{y}$. The mixing is arising as much or more from high-frequency wind fluctuations than it is from turbulence associated with baroclinic instability.

Ageostrophic forcing from eddy PV fluxes and wind stresses balances an equatorward surface flow, and its divergence lifts isopycnals and induces outcrops. Pressure forcing balances a poleward flow, which acts to flatten isopycnals and limit outcropping. If the strength of the ageostrophic upwelling increased, for example by stronger Ekman divergences, then more isopycnals would outcrop. This increase in outcrops could induce a stronger poleward geostrophic transport. The balance between ageostrophic and geostrophic streamfunctions may be attained via the level of isopycnal outcropping. Future work should investigate this balance.

The Drake Passage may be open to circumpolar flow in vertical coordinates, but in isopycnal coordinates it has been shown that there are very few unblocked latitude circles. The large net pressure gradients found in the Drake Passage latitudes in Figure (4-5) are largely supported by discontinuities due to isopycnal outcroppings in the eastern Weddell Sea (Figure 4-6). Integrating the zonal momentum balance along pressure contours, as opposed to along latitude circles, limits intersections with fronts and lateral boundaries, and thus flattens the surface zone "topography". The streamline with the most unbroken latitude circles has an average latitude of $57.8^{0} \mathrm{~S}$. The surface zone "topography" following this streamline is relatively flat (compare Figure (4-16) to integration around the $59.6^{0} \mathrm{~S}$ latitude circle shown in Figure 4-6). The meridional variability of this streamline, however, is substantial (see, for example, the streamlines in Figure 3-2), and the zonal pressure gradient does not vanish upon integration along it (i.e. $\oint p_{x} d z \neq 0$ ). In the Southern Ocean it is not appropriate to approximate the along stream momentum balance as the zonal momentum balance in order to limit the influence of the zonal pressure forcing. Furthermore, rotating 

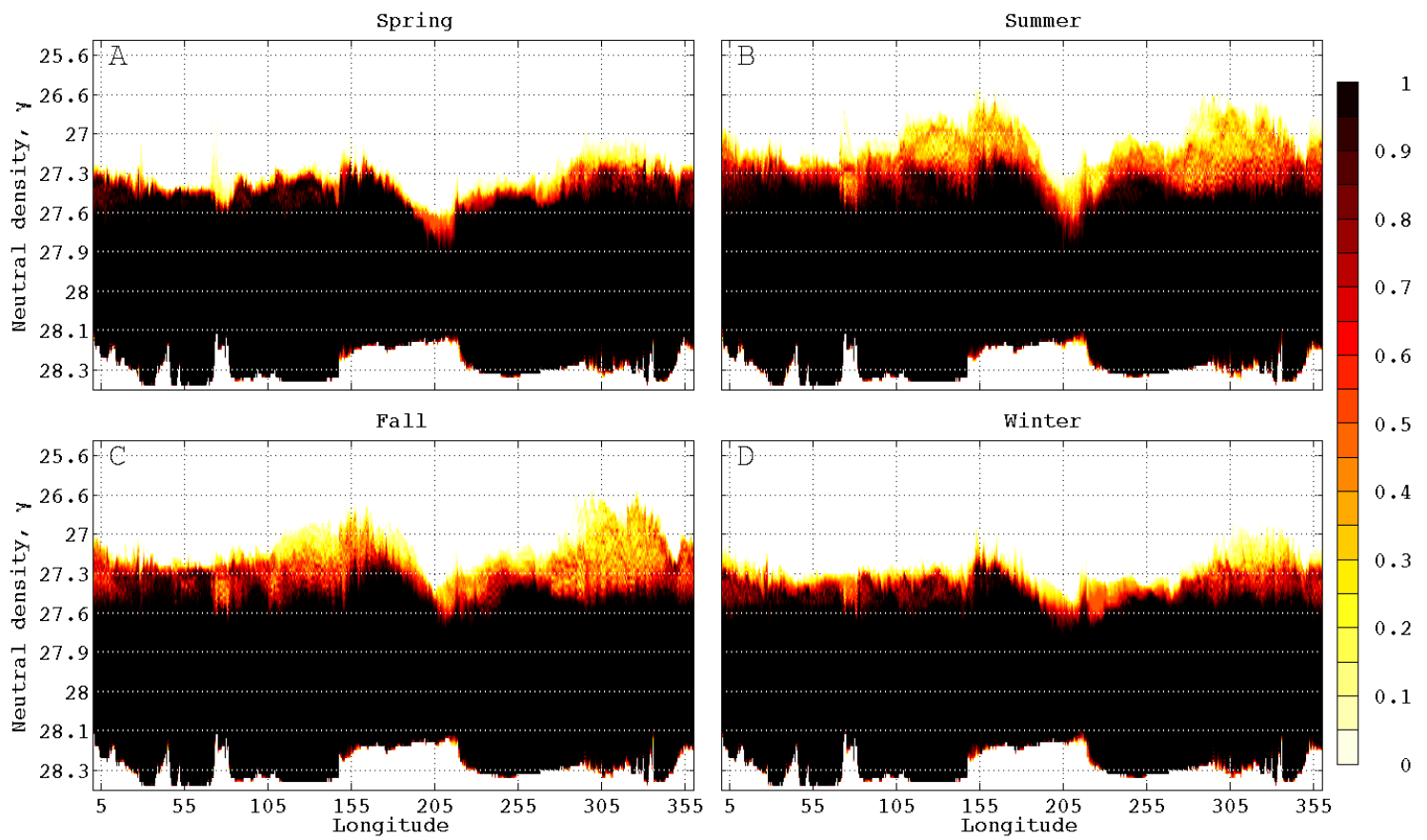

Figure 4-16: The probability that an isopycnal layer exists on a circumpolar streamline in the Southern Ocean state estimate. The average latitude of the streamline plotted is $57.8^{0} \mathrm{~S}$. A value of one means that for the given longitude and season, the isopycnal layer is present at all times on this streamline.

the momentum equations into an across and along stream direction would not cause the geostrophic component of the across streamline overturning circulation to vanish. This is because ACC streamlines do not follow density contours, and correlations arise between the geostrophic velocity and the isopycnal layer thicknesses (Figure 4-17). In other words, though $\oint p_{s} d s$ may vanish, $\oint \gamma_{0} \gamma_{z}^{-1} p_{s} d s$ is likely to be finite.

The state estimate solution is drifting, and as a result the neutral density surfaces are moving in $z$-space over the seasonal averages. One spurious effect of this drift is that it appears there are buoyancy sources in the abyss. This drift may, unfortunately, introduce error in the magnitude of the forcing components. It does not, however, change the lowest order zonal momentum balance found in this thesis.

The state estimate drift is likely a result of inconsistent initial and boundary conditions. It is desirable to eliminate this drift, and then re-evaluate the zonal momentum balance. Future work could use the adjoint machinery to eliminate the drift by introducing constraints in the cost function. The drift found in this eddy permitting state 


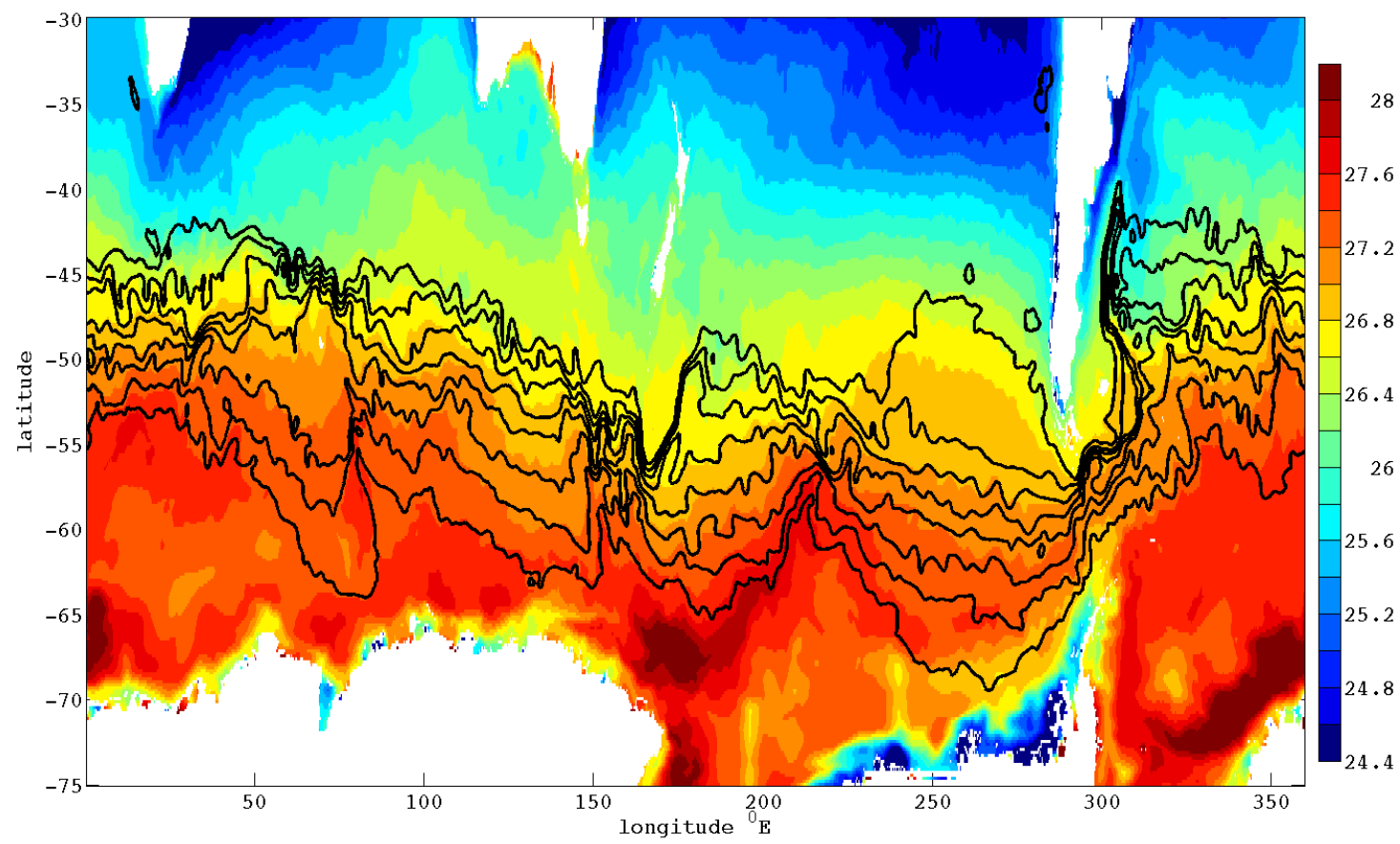

Figure 4-17: Vertically integrated transport streamlines plotted on top of surface neutral density. ACC streamlines do not follow density contours, and thus one should expect geostrophic buoyancy transports to be significant even if geostrophic mass transports vanish (i.e. though $\oint p_{s} d s$ may vanish, $\oint \gamma p_{s} d s$ is likely to be finite).

estimate is, however, also found in the coarse resolution state estimate produced in Forget (2008). The state estimate of Forget (2008) was used to derive the northern boundary conditions and to derive the first guess initial conditions. It would be wise to first reduce the drift in coarse resolution state estimates, as these simulations use less computational resources. The drift is significant in the abyss where eddy resolution is less vital. Understanding the differences between the abyssal force balance in an equilibrated and drifting coarse resolution state estimate will shed light on how things will vary in a high resolution state estimate.

\section{Implications for Idealized Models}

Andrews and McIntyre (1976) first introduced the residual streamfunction to understand the circulation of the atmosphere. This streamfunction can be written as

$$
\Psi^{*}=\bar{\Psi}+\hat{\Psi}
$$


where $\bar{\Psi}$ is the overturning circulation for the Eulerian mean flow, and $\hat{\Psi}$ is the circulation associated with eddy dynamics (Plumb and Ferrari, 2005). It is theorized that the Eulerian mean flow is set by mechanical forcing, while the eddy circulation is governed by turbulent dynamics. These two circulations largely cancel each other, making $\Psi^{*}$ a small residual. This balance has proven appropriate in the "Middleworld" region of the atmosphere where isentropes do not intersect the Earth's surface. In the region where atmospheric isentropes do intersect topography, the surface zone or "Underworld", pressure forcing becomes significant (Koh and Plumb, 2004; Schneider, 2005).

The success of using the residual formulation of Equation (4.25) to describe the atmospheric Middleworld has prompted its application to the Southern Ocean meridional overturning circulation (e.g. Marshall and Radko (2003); Olbers and Visbeck (2005)). It has been shown (Figure 4-6), however, that the ocean is much more analogous to the atmospheric surface zone than the atmospheric Middleworld. Thus, as in the atmospheric surface zone, pressure forcing cannot be neglected. It is therefore important to note that the Eulerian streamfunction is strongly geostrophic, $\bar{\Psi}=\bar{\Psi}_{E k}+\bar{\Psi}_{g}=-\overline{f^{-1} \tau_{0}}+\overline{f^{-1} p_{x}}$. Similarly, decomposition of the residual velocity leads to $\overline{v h}=\overline{v_{E k}} \bar{h}+\overline{v_{g}} \bar{h}+\overline{v^{\prime} h^{\prime}}$. This balance was evaluated in Chapter 3 . The eddy transport, $\overline{v^{\prime} h^{\prime}}$, was plotted in Figure (3-13). It is now apparent that the mean flow plotted in Figure (3-14) was a combination of the Ekman transport and the geostrophic transport, $\left(\overline{v_{E k}}+\overline{v_{g}}\right) \bar{h}$. A conclusion of this work is that pressure forcing cannot be neglected when theorizing on the Southern Ocean overturning circulation. 


\section{Chapter 5}

\section{Conclusions and Future Work}

\subsection{The Southern Ocean State Estimate}

A modern general circulation model of the Southern Ocean with one-sixth of a degree resolution has been fit to the observed ocean in a weighted least squares sense (see Section 1.2). Reducing the misfit of this eddy permitting state estimate to the observations via the adjoint method is a big step towards the goal of producing global eddy resolving state estimates. Equally important, the estimate provides a useful data set to illuminate the Southern Ocean dynamics (see Section 2.3 and 3.4). Much of what is known in regards to the Southern Ocean circulation comes from models such as the Fine Resolution Antarctic Model (FRAM: e.g. Saunders and Thompson (1993)) and the Ocean Circulation and Climate Advanced Model (OCCAM: e.g. Lee et al. (2007)). These models "put dynamics to climatology" through unphysical relaxation terms. The Southern Ocean state estimate produced in this thesis puts realistic physics to all available modern ocean observations. Because there are no unphysical relaxation terms, and because climatology is suspect in the Southern Ocean, the state estimate produced is a superior product for evaluating dynamics.

The state estimate produced, however, is a work in progress. It has not yet fully converged; Figure (2-1) suggests the cost can be further reduced with subsequent iterations. Computational resources have been secured to ensure that these iterations will be carried out. The state estimate is planned to be improved in several other 
ways. For example, its temporal duration is to be extended through 2007 and more control parameters are to be added. Of course, it is also the hope that additional observations will be attained. The Southern Ocean state estimate will benefit from the continually occurring improvements to the ECCO state estimation infrastructure. These improvements range from better forward model parameterizations to a better determination of the prescribed observational uncertainty. Future work may involve a recalculation of the dynamical balances and transports analyzed in this thesis using a more converged iteration.

The Southern Ocean state estimate produced provides a reference data set that can be used for dynamical analyses. It can also be used to evaluate free-running circulation models, and to put field experiments into a broader context. Research efforts analyzing the state estimate include an assessment of the effect of stirring by mesoscale eddies, which is being carried out by the Marshall group at MIT (Abernathey et al., 2008). I. Cerovecki, SIO-UCSD, has been analyzing water mass formation rates in the state estimate (Cerovecki et al., 2008). A proposal to carry out a carbon-cycle model by T. Ito (Colorado State University) using the estimated dynamical state has been funded for three years by NASA. J. Ledwell, WHOI, is using the solution to guide future observational programs; in the context of the DIMES project (http://dimes.ucsd.edu) passive tracers have been released in the state estimate to simulate the evolution of a dye release. And of course, several aspects of the Southern Ocean's dynamics have been investigated in this thesis.

The near-term plans for the Southern Ocean state estimate are to further its development and quality, and to continue with its analysis. Subjects to be investigated include an evaluation of where mixing is important, and of the energy budget. It is likely that numerous future studies analyzing the state estimate fields will be carried out. Regarding long-term plans, the hope is that this estimate of the modern Southern Ocean will be synthesized into a high-resolution state estimate of the global ocean. 


\subsection{Analysis of the Zonal Momentum Balance}

The Southern Ocean state estimate is largely consistent with the individual observations (Chapter 2). Furthermore, the time mean integral fluxes in the state estimate are largely consistent with recent static inverse estimates (Chapter 3). No gross inconsistencies have been found, giving confidence to the force balances deduced from the solution. The zonal momentum force balance has been found to have a significant geostrophic component. This transport component, however, is neglected in many current idealized theories of the Southern Ocean circulation.

The current consensus, despite a few dissenters (e.g. Warren et al. (1996)), on how one should theorize about the dynamics of the Southern Ocean is summed up in this quote from Marshall and Radko (2006):

the absence of zonal boundaries in the southern ocean has enabled the meteorologists zonal-average residual-mean theory to be applied to develop conceptual models that draw together in suggestive ways the relative roles of wind, buoyancy and eddy forcing in setting the stratification, transport and overturning circulation of the Southern Ocean.

The meteorologists zonal-average residual-mean theories being referred to do not include the effect of pressure forces, as these vanish by periodicity in unblocked integrals. Indeed, the word "pressure" is not found in Marshall and Radko (2006). Recently it has been shown, however, that the meteorologists theories must include the effects of pressure forcing on any isentrope that may intersect horizontal boundaries (Koh and Plumb, 2004; Schneider, 2005). This, of course, is the case for the ocean as well. Using the state estimate, this thesis showed that most ocean isopycnal layers do intersect boundaries. As a result, pressure forcing appears at lowest order in the zonal momentum balance. It follows that the zonal-average residual-mean theories of the Southern Ocean circulation must include geostrophic flows.

Surface poleward heat transports are found to dominate the buoyancy budget north of the Drake Passage latitudes. An Ekman transport, however, drives buoyancy equatorward. Popular theories of the nature of Marshall and Radko (2006) 
attribute the net poleward heat flux as an overpowering of the Ekman heat flux by an ageostrophic Stokes drift. It was found that near surface eddies actually work in concert with the equatorward Ekman transport, and that pressure forces acting on the most buoyant waters are responsible for balancing the poleward geostrophic transport that allows the buoyancy budget to be closed. Pressure forcing is also of lowest order importance at depth. Indeed, the Southern Ocean's characteristic multiple cell overturning circulation is primarily in geostrophic balance.

It is true that the net geostrophic volume transport vanishes when integrated zonally in z-space: $\oint v_{g} d x=0$, where $v_{g} \equiv\left(f \rho_{0}\right)^{-1} p_{x}$. Correlation terms, for example the net geostrophic temperature transport, do not necessarily vanish: $\oint T v_{g} d x \neq 0$. Thus the net geostrophic volume transport is likely to be finite when zonally integrated in temperature space. This is true of any material surface in the ocean, and in this thesis it has been shown to be true with neutral density surfaces. The transformed Eulerian mean (or residual mean) circulation is volume weighted and mapped to isopycnal classes, and this keeps its geostrophic component from vanishing upon zonal integration: $\oint\left(\rho_{0} \rho_{z}^{-1}\right) v_{g} d x \neq 0$. That the geostrophic residual mean circulation in the Southern Ocean is not just finite, but is of lowest order importance, is a primary result of this thesis. The buoyancy transport by the geostrophic circulation is also found to be of lowest order importance, and thus idealized Southern Ocean theories need not invoke eddy transport mechanisms to counter the strong buoyancy transport by the wind driven Ekman flow.

Using an eddy-permitting state estimate, the Southern Ocean's zonal momentum balance in isopycnal space has, for the first time, been analyzed. It was found that eddy surface buoyancy fluxes and wind forced eddy potential vorticity fluxes are of lowest order importance. There is concern that parameterization schemes meant to represent these effects are suspect due to methods implemented to deal with the steep isopycnal slopes found near the oceans surface. That these terms have been found to be significant to the dynamics suggests that effort should be made to improve near surface parameterizations. Most importantly, it was found that pressure forcing is of lowest order importance to the meridional overturning force balance of the Southern 
Ocean.

A major outstanding problem in physical oceanography is determining what sets the ACC's time-mean zonal transport. Theories of what sets the baroclinic component of this transport have relied on a balance between eddies and wind forcing. These theories state that the wind lifts isopycnals, while eddies and mixing act to flatten them. The meridional isopycnal tilt, and thus the zonal velocity vertical shear, is set through this balance. The idea has been posed that the ACC may be at a "saturation" point where isopycnal tilts cannot be further steepened even if the wind were to increase (or flattened due to a slight decrease of the wind; Marshall and Radko (2003); Hallberg and Gnanadesikan (2006)). This thesis shows, however, that eddies do not act to flatten the isopycnals of the ACC.

The realization that geostrophic flows are balancing the wind driven transport allows new speculation as to what sets the ACC's time-mean zonal transport. It is known that the ACC's zonal transport is primarily in geostrophic balance, and that the meridional pressure gradient is set largely from a 1.5 meter drop (from north to south) in mean sea surface height (Olbers et al., 2004). An increase in zonal winds would result in an increase in the northward Ekman transport, and unless this transport is compensated, an increase in this sea surface height gradient. The Ekman transport would also bring more dense waters equatorward, and thus further increase the pressure difference across the ACC. Determining what sets the meridional overturning circulation therefore allows one to infer what is setting the mean ACC zonal transport. This thesis has only elucidated the force balance of the meridional overturning circulation, however, not what sets it.

One may speculate what sets the meridional overturning circulation. Taking an oceanographic point of view excuses explanation of the atmospheric state, leaving one to explain only the eddy forcing and the zonal pressure gradient. As to the eddy driven transport, one may speculate that this component is proportional to the PV input by surface forcing. The abyssal geostrophic transport is likely proportional to the formation rates of North Atlantic Deep Water and Antarctic Bottom Water. One may also speculate that the equatorward geostrophic transport of Antarctic 
Intermediate Water is set by water mass formation rates. It is unlikely, however, that one may relate the massive $(\sim 30 \mathrm{~Sv})$ poleward geostrophic transport of the most buoyant waters to atmospheric buoyancy forcing. This transport is likely related to the intrinsic dynamics of the ACC system. Thus it is at this point where the question returns (full circle) to what sets the zonal ACC strength. Inferring what sets a balance from analysis of a system in balance is difficult, if not impossible. Future work could analyze an ACC "spin-up" (or spin-down) problem in order to shed determine what sets this current systems strength. It may also be possible to shed light on this question through analysis of the Southern Ocean state estimate's variability. 


\section{Bibliography}

Abernathey, R., Marshall, J., Mazloff, M., and Shuckburgh, E. (2008). Studies of enhanced isopycnal mixing at deep critical levels in the southern ocean. in preperation.

Adcroft, A., Campin, J.-M., Heimbach, P., Hill, C., and Marshall, J. (2006). Mitgcm user's manual. (online documentation), MIT / EAPS, Cambridge, MA 02139, USA. http://mitgcm.org/.

Adcroft, A. and Marshall, D. (1998). How slippery are piecewise-constant coastlines in numerical ocean models? Tellus Series A, 50:95-+.

Andrews, D., Holton, J., and Leovy, C. (1987). Middle atmosphere dynamics. Academic Press, London.

Andrews, D. G. and McIntyre, M. E. (1976). Planetary waves in horizontal and vertical shear: The generalized Eliassen-Palm relation and the mean zonal acceleration. Journal of Atmospheric Sciences, 33:2031-2048.

Arakawa, A. and Lamb, V. (1977). Computational design of the basic dynamical processes of the ucla general circulation model. Methods in Computational Physics, $17: 174-267$.

Bi, D., Hirst, A., O'Farrell, S., Phipps, S., Marsland, S., Roberts, J., Heil, P., Tseitkin, F., and Alves, O. (2008). Development of AusCOM: the Australian coupled ocean and sea ice model. In AMOS 2008: 15th National Australian Meteorological and Oceanographic Society (AMOS) Conference in conjunction with the Australian 
Wind Engineering Society (AWES) and Meteorological Society of New Zealand: atmospheric and oceanic extremes, page 16. AMOS, Deakin University, Waterfront Campus, Geelong, Victoria, Australia.

Bryden, H. L. and Pillsbury, R. D. (1977). Variability of Deep Flow in the Drake Passage from Year-Long Current Measurements. Journal of Physical Oceanography, $7: 803-810$.

Cerovecki, I., Talley, L., and Mazloff, M. (2008). Water mass transformation in the southern ocean: Subantarcic mode water and antarctic intermediate water formation. In preperation.

Chelton, D. B., deSzoeke, R. A., Schlax, M. G., Naggar, K. E., and Siwertz, N. (1998). Geographical variability of the first baroclinic rossby radius of deformation. Journal of Physical Oceanography, 28(3):433-460.

Chelton, D. B., Schlax, M. G., Freilich, M. H., and Milli, R. F. (2004). Satellite measurements reveal persistent small-scale features in ocean winds. Science, 303:978983.

Conkright, M. E., Locarnini, R., Garcia, H. E., O’Brien, T., Boyer, T., Stephens, C., and Antonov, J. (2002). World ocean atlas 2001: Objective analyses, data statistics, and figures,cd-rom documentation. Technical report, Natl. Oceanogr.Data Cent., Silver Spring,Md.

Cunningham, S. A., Alderson, S. G., King, B. A., and Brandon, M. (2003). Transport and variability of the Antarctic Circumpolar Current in drake passage. J. Geophys. Res., 108(C5).

de Szoeke, R. A. and Bennett, A. F. (1993). Microstructure Fluxes across Density Surfaces. Journal of Physical Oceanography, 23:2254-2264.

Döös, K. and Webb, D. (1994). The deacon cell and the other meridional cells of the Southern Ocean. J. Phys. Oceanogr., 24:429-442. 
Fekete, B. M., Vöröosmarty, C. J., and Grabs, W. (2002). High-resolution fields of global runoff combining observed river discharge and simulated water balances. Global Biogeochemical Cycles, , 16(3).

Ferreira, D., Marshall, J., and Heimbach, P. (2005). Estimating eddy stresses by fitting dynamics to observations using a residual-mean ocean circulation model and its adjoint. Journal of Physical Oceanography, 35(10):1891-1910.

Forget, G. (2008). A comprehensive ocean atlas for the recent years. In Preperation.

Forget, G. and Wunsch, C. (2007). Estimated Global Hydrographic Variability. Journal of Physical Oceanography, 37:1997-+.

Fučkar, N. S. and Vallis, G. K. (2007). Interhemispheric influence of surface buoyancy conditions on a circumpolar current. Geophysical Research Letters, 34:14605-+.

Ganachaud, A. and Wunsch, C. (2000). Improved estimates of global ocean circulation, heat transport and mixing from hydrographic data. Nature, 408(6811):453457.

Ganachaud, A. and Wunsch, C. (2003). Large-scale ocean heat and freshwater transports during the world ocean circulation experiment. Journal of Climate, 16.

Garabato, A. C. N., Polzin, K. L., King, B. A., Heywood, K. J., and Visbeck, M. (2004). Widespread intense turbulent mixing in the Southern Ocean. Science, 303:210-213.

Gebbie, G. (2004). Subduction in an Eddy-resolving State Estimate of the Northeast Atlantic Ocean. PhD thesis, Massachusetts Institute of Technology / Woods Hole Oceanographic Institution Joint Program in Oceanography.

Gebbie, G. A., Heimbach, P., and Wunsch, C. (2006). Strategies for nested and eddyresolving state estimation. J. Geophys. Res., 111. doi:10.1029/2005JC003094. 
Gent, P. R., Craig, A. P., Bitz, C. M., and Weatherly, J. W. (2002). Parameterization improvements in an eddy-permitting ocean model for climate. Journal of Climate, 15(12):1447-1459.

Gent, P. R. and McWilliams, J. C. (1990). Isopycnal mixing in ocean circulation models. J. Phys. Oceanogr., 20(1):150-155.

Giering, R. and Kaminski, T. (1998). Recipes for adjoint code construction. ACM Transactions on Mathematical Software, 24(4):437-474.

Gilbert, J. C. and Lemaréchal, C. (1989). Some numerical experiments with variablestorage quasi-Newton algorithms. Math. Program., 45:407-435.

Gill, A. (1968). A linear model of the Antarctic Circumpolar Current. Journal of Fluid Mechanics, 32:465-488.

Gill, P. E., Murray, W., and Wright, M. H. (1986). Practical Optimization. Academic Press, San Diego, CA.

Gille, S. T. (1995). Dynamics of the Antarctic Circumpolar Current: Evidence for Topographic Effects from Altimeter Data and Numerical Model Output. PhD thesis, MIT-WHOI Joint Program, Cambridge MA.

Gnanadesikan, A. and Hallberg, R. (2000). On the relationship of the circumpolar current to southern hemisphere winds in large-scale ocean models. J. Phys. Oceanogr., 30:2013-2034.

Gong, D. and Wang, S. (1999). Definition of Antarctic oscillation index. Geophysical Research Letters, 26:459-462.

Gould, J. and the Argo Steering Team (2004). Argo profiling floats bring new era of in situ ocean observations. EOS Trans. Am. Geophys. Un., 85(19):190-191.

Gouretski, V. V. and Koltermann, K. P. (2004). WOCE Global Hydrographic Climatology. Available at http://www.bsh.de/de/Produkte/Buecher/Berichte/index.jsp. 
Hallberg, R. and Gnanadesikan, A. (2006). The role of eddies in determining the structure and response of the wind-driven southern hemisphere overturning: Results from the modeling eddies in the Southern Ocean (meso) project. Journal of Physical Oceanography, 36(12):2232-2252.

Heimbach, P. (2008). The mitgcm/ecco adjoint modeling infrastructure. CLIVAR Exchanges, 13(1).

Heimbach, P., Campin, J., Dutkiewicz, S., Evangelinos, C., Ferreira, D., Forget, G., Hill, C., Hill, E., Losch, M., Marshall, J., Mazloff, M., Menemenlis, D., Ponte, R., and Wunsch, C. (2008). The MITgcm/ECCO adjoint modelling infrastructure. CLIVAR Exchanges, 44.

Heimbach, P., Ponte, R., Evangelinos, C., Forget, G., Mazloff, M., Menemenlis, D., Vinogradov, S., and Wunsch, C. (2006). Combining altimetric and all other data with a general circulation model. In 15 Years of Progress in Radar Altimetry Symposium, volume SP-614. ESA Special Publication.

Hibler, III, W. D. (1980). Modeling a variable thickness sea ice cover. Mon. Weather Rev., 1:1943-1973.

Hoteit, I., Cornuelle, B., Kohl, A., and Stammer, D. (2005). Treating strong adjoint sensitivities in tropical eddy-permitting variational data assimilation. $Q . J . R$. Meteorol. Soc., 131:3659-3682.

Hughes, C. W. and Wilson, C. (2007). Wind work on the geostrophic circulation: an observational study of the effect of small scales in the wind stress. Submitted for publication.

Hughes, C. W., Woodworth, P. L., Meredith, M. P., Stepanov, V., Whitworth, T., and Pyne, A. R. (2003). Coherence of Antarcticsea levels, southern hemisphere annular mode, and flow through drake passage. Geophysical Research Letters, 30(9).

Ito, T. and Marshall, J. (2007). Control of lower limb circulation in the Southern Ocean by diapycnal mixing and mesoscale eddy transfer. submitted to JPO. 
Jackett, D. R. and McDougall, T. J. (1997). A Neutral Density Variable for the World's Oceans. Journal of Physical Oceanography, 27:237-263.

Johnson, G. C. and Bryden, H. L. (1989). On the size of the Antarctic Circumpolar Current. Deep-Sea Research, 36:39-53.

Kalnay, E. and coauthors (1996). The NCEP/NCAR 40-year reanalysis project. Bull. Amer. Meteor. Soc., 77:431-437.

Killworth, P. D. and Nanneh, M. M. (1994). Isopycnal Momentum Budget of the Antarctic Circumpolar Current in the Fine Resolution Antarctic Model. Journal of Physical Oceanography, 24:1201-+.

Koh, T.-Y. and Plumb, R. A. (2004). Isentropic zonal average formalism and the near-surface circulation. Quarterly Journal of the Royal Meteorological Society, 130:1631-1653.

Köhl, A. and Willebrand, J. (2002). An adjoint method for the assimilation of statistical characteristics into eddy-resolving ocean models. Tellus Series A, 54:406-+.

Kuo, A., Plumb, R. A., and Marshall, J. (2005). Transformed Eulerian-Mean Theory. Part II: Potential Vorticity Homogenization and the Equilibrium of a Wind- and Buoyancy-Driven Zonal Flow. Journal of Physical Oceanography, 35:175-+.

Large, W. and Pond, S. (1982). Sensible and latent-heat flux measurements over the ocean. Journal of Physical Oceanography, 12(5):464-482.

Large, W. G., McWilliams, J. C., and Doney, S. (1994). Oceanic vertical mixing: A review and a model with a nonlocal boundary layer parameterization. Rev. Geophysics, 32:363.

Large, W. G. and Yeager, S. G. (2008). The Global Climatology of an Interannually Varying Air-Sea Flux Data Set. Submitted to Climate Dynamics.

Lee, M.-M. and Coward, A. (2003). Eddy mass transport for the Southern Ocean in an eddy-permitting global ocean model. Ocean Modelling, 5(3):249-266. 
Lee, M.-M., Nurser, A. J. G., Coward, A. C., and de Cuevas, B. A. (2007). Eddy Advective and Diffusive Transports of Heat and Salt in the Southern Ocean. Journal of Physical Oceanography, 37:1376--+.

Levitus, S., Burgett, R., and Boyer, T. (2001). World ocean atlas 2001. Technical report, U.S. Department of Commerce, Washington, D.C.

Losch, M. and Heimbach, P. (2007). Adjoint Sensitivity of an Ocean General Circulation Model to Bottom Topography. Journal of Physical Oceanography, 37:377-+.

Losch, M. and Wunsch, C. (2003). Bottom topography as a control variable in an ocean model. Journal of Atmospheric and Oceanic Technology, 20(8):1685-1696.

Lu, Y., Ueyoshi, K., Kohl, A., Remy, E., Lorbacher, K., and Stammer, D. (2002). Input data sets for the ecco global $1^{\circ}$ woce synthesis. The ECCO Report Series, 18. Available at http://www.ecco-group.org/reports.html.

Lumpkin, R. and Speer, K. (2007). Global Ocean Meridional Overturning. Journal of Physical Oceanography, 37:2550-+.

MacDonald, A. M. and Wunsch, C. (1996). An estimate of global ocean circulation and heat fluxes. Nature, 382:436-439.

Marsh, R., Nurser, A. J. G., Megann, A. P., and New, A. L. (2000). Water mass transformation in the Southern Ocean of a global isopycnal coordinate gcm. Journal of Physical Oceanography, 30(5):1013-1045.

Marshall, J., Adcroft, A., Hill, C., Perelman, L., and Heisey, C. (1997a). A finitevolume, incompressible Navier-Stokes model for studies of the ocean on parallel computers. J. Geophys. Res., 102(C3):5753-5766.

Marshall, J., Hill, C., Perelman, L., and Adcroft, A. (1997b). Hydrostatic, quasihydrostatic and non-hydrostatic ocean modeling. J. Geophys. Res., 102(C3):57335752. 
Marshall, J., Olbers, D., Ross, H., and Wolfgladrow, D. (1993). Potential vorticity constraints on the dynamics and hydrography of the southern-ocean. J. Phys. Oceanogr., 23(3):465-487.

Marshall, J. and Radko, T. (2003). Residual-Mean Solutions for the Antarctic Circumpolar Current and Its Associated Overturning Circulation. Journal of Physical Oceanography, 33:2341-+.

Marshall, J. and Radko, T. (2006). A model of the upper branch of the meridional overturning of the southern ocean. Progress in Oceanography, 70:331-345.

Martinson, D. G., Killworth, P. D., and Gordon, A. L. (1981). A Convective Model for the Weddell Polynya. Journal of Physical Oceanography, 11:466-488.

Mazloff, M. R. (2006). Production and Analysis of a Southern Ocean State Estimate. Massachusetts Institute of Technology and the Woods Hole Oceanographic Institution, Cambridge, Massachusetts.

McIntosh, P. C. and McDougall, T. J. (1996). Isopycnal averaging and the residual mean circulation. Journal of Physical Oceanography, 26(8):1655-1660.

Motoi, T., Ono, N., and Wakatsuchi, M. (1987). A Mechanism for the Formation of the Weddell Polynya in 1974. Journal of Physical Oceanography, 17:2241-2247.

Nan, S. and Li, J. (2003). The relationship between the summer precipitation in the Yangtze River valley and the boreal spring Southern Hemisphere annular mode. Geophysical Research Letters, 30(24):240000-1.

Nierenberg, W., Radok, R., and Phillips, O. (1970). Physical and chemical data from the Southern Ocean between Australia and Antarctica, USNS ELTANIN Cruise 41, 1969-1970. SIO ref. 10-72-19, Scripps Institute of Oceanography, University of California, San Diego.

NOAA (1988.). Data announcement 88-mgg-02: Digital relief of the surface of the earth. National Geophysical Data Center, Boulder, Colorado. 
Olbers, D., Borowski, D., Volker, C., and Wolff, J.-O. (2004). The dynamical balance, transport and circulation of the Antarctic Circumpolar Current. AntarcticScience, 16(4):439-470.

Olbers, D. and Eden, C. (2003). A simplified general circulation model for a baroclinic ocean with topography. part i: Theory, waves, and wind-driven circulations. Journal of Physical Oceanography, 33(12):2719-2737.

Olbers, D. and Visbeck, M. (2005). A model of the zonally averaged stratification and overturning in the Southern Ocean. Journal of Physical Oceanography, 35(7):11901205.

Orsi, A. H., Whitworth, T., and Nowlin, W. D. (1995). On the meridional extent and fronts of the Antarctic Circumpolar Current. Deep Sea Research, 42(5):641-673.

Patra, P. K., Maksyutov, S., Ishizawa, M., Nakazawa, T., Takahashi, T., and Ukita, J. (2005). Interannual and decadal changes in the sea-air co2 flux from atmospheric co2 inverse modeling. Global Biogeochemical Cycles, 19(4):Art. No. GB4013.

Pedlosky, J. (1987). Geophysical Fluid Dynamics. Springer-Verlag, Berlin-HeidelbergNew York., 2 edition.

Pedlosky, J. (1996). Ocean Circulation Theory. Springer, New York. 464 pp.

Penduff, T., Barnier, B., Kerbiriou, M.-A., and Verron, J. (2002). How topographic smoothing contributes to differences between the eddy flows simulated by sigma and geopotential-coordinate models. J. Phys. Oceanogr., 32:122-137.

Pickard, G. L., Emery, W. J., and Talley, L. D. (2008). Descriptive Physical Oceanography. Elsevier, Amsterdam.

Plumb, R. A. and Ferrari, R. (2005). Transformed Eulerian-Mean Theory. Part I: Nonquasigeostrophic Theory for Eddies on a Zonal-Mean Flow. Journal of Physical Oceanography, 35:165-+. 
Redi, M. H. (1982). Oceanic isopycnal mixing by coordinate rotation. J. Phys. Oceanogr., 12:1154-1158.

Reid, J. (1994). On the total geostrophic circulation of the North Atlantic Ocean: flow patterns, tracers and transports. Progr. in Oceanogr., 33:1-92.

Rintoul, S. R., Hughes, C. W., and Olbers, D. (2001). The Antarctic Circumpolar Current system. In Siedler, G., Church, J., and Gould, J., editors, Ocean Circulation and Climate, pages 271-302. Academic Press.

Rintoul, S. R. and Sokolov, S. (2001). Baroclinic transport variability of the Antarctic Circumpolar Current south of Australia (WOCE repeat section SR3). Journal of Geophysical Research, 106:2795-2814.

Samelson, R. M. (1999). Geostrophic circulation in a rectangular basin with a circumpolar connection. Journal Of Physical Oceanography, 29.

Samelson, R. M. (2004). Simple Mechanistic Models of Middepth Meridional Overturning. Journal of Physical Oceanography, 34:2096-+.

Saunders, P. and Thompson, S. (1993). Transport, heat and freshwater fluxes within a diagnostic model (fram). J. Phys. Oceanogr., 23:452-464.

Schneider, T. (2005). Zonal momentum balance, potential vorticity dynamics, and mass fluxes on near-surface isentropes. Journal of the Atmospheric Sciences, $62(6): 1884$.

Schroder, M. and Fahrbach, E. (1999). On the structure and the transport of the eastern Weddell Gyre. Deep Sea Research Part II: Topical Studies in Oceanography, 46:501-527.

Sloyan, B. M. and Rintoul, S. R. (2001). Circulation, renewal, and modification of Antarcticmode and intermediate water. J. Phys. Oceanogr., 31(4):1005-1030.

Smith, W. and Sandwell, D. (1997). Global sea floor topography from satellite altimetry and sparse shipboard bathymetry. Science, 277:1956-1961. 
Speer, K., Rintoul, S. R., and Sloyan, B. (2000). The Diabatic Deacon Cell. Journal of Physical Oceanography, 30:3212-3222.

Stammer, D., Ueyoshi, K., Kohl, A., Large, W. G., Josey, S. A., and Wunsch, C. (2004). Estimating air-sea fluxes of heat, freshwater, and momentum through global ocean data assimilation. J. Geophys. Res., 109(C5).

Stammer, D., Wunsch, C., Giering, R., Eckert, C., Heimbach, P., Marotzke, J., Adcroft, A., Hill, C. N., and Marshall, J. (2003). Volume, heat, and freshwater transports of the global ocean circulation 1993-2000, estimated from a general circulation model constrained by World Ocean Circulation Experiment (WOCE) data. J. Geophys. Res., 108(C1):3007-3029.

Stephens, C., Antonov, J., Boyer, T., Conkright, M., Locarnini, R., O'Brien, T., and Garcia, H. (2001). World ocean atlas 2001.

Stewart, R. H. (2005). Introduction to Physical Oceanography. Open Source Online Textbook: http://oceanworld.tamu.edu/resources/ocng_textbook/contents.html.

Stommel, H. (1957). A survey of ocean current theory. Deep-Sea Res., 4:149-84.

Sultan, E., Mercier, H., and Pollard, R. (2007). An inverse model of the large scale circulation in the South Indian Ocean. Progress in Oceanography, 74:71-94.

Talley, L. D. (2003). Shallow, Intermediate, and Deep Overturning Components of the Global Heat Budget. Journal of Physical Oceanography, 33:530-560.

Talley, L. D., Reid, J. L., and Robbins, P. E. (2003). Data-based meridional overturning streamfunctions for the global ocean. Journal of Climate, 16(19):3213-3226.

Tanaka, Y. and Hasumi, H. (2008). Injection of Antarctic Intermediate Water into the Atlantic subtropical gyre in an eddy resolving ocean model. Geophysical Research Letters, 35:11601-+. 
Tansley, C. E. and Marshall, D. P. (2001). Flow past a cylinder on a beta-plane, with application to gulf stream separation and the Antarctic Circumpolar Current. J. Phys. Oceanogr., 31:3274-3283.

Tomczak, M. and Liefrink, S. (2005). Interannual variations of water mass volumes in the Southern Ocean. Journal of Atmospheric and Ocean Science, 10(1):31-42.

Toole, J. M., Polzin, K. L., and Schmitt, R. W. (1994). Estimates of Diapycnal Mixing in the Abyssal Ocean. Science, 264:1120-1123.

Vallis, G. K. (2000). Large-Scale Circulation and Production of Stratification: Effects of Wind, Geometry, and Diffusion. Journal of Physical Oceanography, 30:933-954.

Vallis, G. K. (2006). Atmospheric and Oceanic Fluid Dynamics. Atmospheric and Oceanic Fluid Dynamics, by Geoffrey K. Vallis, pp. 770. Cambridge University Press, November 2006. ISBN-10: 0521849691. ISBN-13: 9780521849692, Cambridge.

Warren, B. A., LaCasce, J. H., and Robbins, P. E. (1996). On the obscurantist physics of form drag in theorizing about the circumpolar current. J. Phys. Oceanogr, $26: 2297-2301$.

Warren, B. A., LaCasce, J. H., and Robbins, P. E. (1998). Comments on "on the obscurantist physics of "form drag" in theorizing about the circumpolar current"reply. Journal of Physical Oceanography, 28(8):1655-1658.

Webb, D. (1993). A simple model of the effect of the kerguelen plateau on. Geophysical and Astrophysical Fluid Dynamics, 70:57-84.

Whitworth, III, T. and Peterson, R. G. (1985). Volume transport of the Antarctic Circumpolar Current from bottom pressure meaurements. J. Phys. Oceanogr., $15(6): 810-816$.

Wijffels, S. E., Toole, J. M., and Davis, R. (2001). Revisiting the South Pacific subtropical circulation: A synthesis of World Ocean Circulation Experiment observations along $32^{0} \mathrm{~S}$. Journal of Geophysical Research-oceans, 106:19481-19514. 
Wilson, C. and Williams, R. G. (2004). Why Are Eddy Fluxes of Potential Vorticity Difficult to Parameterize? Journal of Physical Oceanography, 34:142-+.

Wolfe, C. and Cessi, P. (2008). Overturning circulation in an eddy-resolving model: The effect of the pole-to-pole temperature gradient. Submitted to the Journal of Physical Oceanography.

Wunsch, C. (1998). The work done by the wind on the oceanic general circulation. J. Phys. Oceanogr., 28(11):2332-2340.

Wunsch, C. (2006). Discrete inverse and state estimation problems : with geophysical fluid applications. Cambridge University Press, Cambridge.

Wunsch, C. and Heimbach, P. (2005). Practical global oceanic state estimation. Physica D.

Wunsch, C. and Heimbach, P. (2006). Decadal changes in the north atlantic meriodional overturning and heat flux. J. Phys. Oceanogr., 36(11):2012-2024. doi:10.1175/JPO2957.1.

Wunsch, C. and Heimbach, P. (2007). Practical global oceanic state estimation. Physica D, 230(1-2):197-208.

Zhai, X. and Greatbatch, R. J. (2007). Wind work in a model of the northwest atlantic ocean. Geophysical Research Letters, 34. 